Representações do grupo de tranças por automorfismos de grupos

\author{
Pavel Jesus Henríquez Pizarro
}


SERVIÇO DE PÓS-GRADUAÇÃO DO ICMC-USP

Data de Depósito:

Assinatura:

\title{
Representações do grupo de tranças por automorfismos de grupos
}

\author{
Pavel Jesus Henríquez Pizarro
}

Orientador: Prof. Dr. Jose Eduardo Prado Pires de Campos

Dissertação apresentada ao Instituto de Ciências Matemáticas e de Computação - ICMC-USP, como parte dos requisitos para obtenção do título de Mestre em Ciências - Matemática . VERSÃO REVISADA

USP - São Carlos

Dezembro de 2011 
Ficha catalográfica elaborada pela Biblioteca Prof. Achille Bassi e Seção Técnica de Informática, ICMC/USP, com os dados fornecidos pelo(a) autor(a)

Henriquez Pizarro, Pavel Jesus
H18 Representações do grupo de tranças por
r
automorfismos de grupos / Pavel Jesus Henriquez
Pizarro; orientador Jose Eduardo Prado Pires de
Campos -- São Carlos, 2011.
123 p.
Dissertação (Mestrado - Programa de Pós-Graduação en
Matemática)-- Instituto de Ciências Matemáticas e
de Computação, Universidade de São Paulo, 2011.
1. Teoria de Tranças. 2. Teoria de Nós. 3.
Topologia. 4. Grupos Livres. 5. Grupoides. I. Prado
Pires de Campos, Jose Eduardo, orient. II. Título.



"Não estamos na terra para sofrer eternamente, mas sim, para que nosso Espírito evolua, por isso a necessidade de tantas provas que muitas vezes nos faz sofrer. Porém, dentro de cada um de nós, existe uma grande força interior, capaz de fazer nos continuar a jornada, enfrentando qualquer obstáculo que apareça. Uma força que nos impulsiona para descoberta que somos capazes de atravessar as tormentas e ir em busca de um novo horizonte. De que se uma pedra nos atinge, saberemos que a dor se fará presente sim, mas também passará, $e$ de que podemos superar o sofrimento e com fé uma nova porta se abrirá. Não esqueção essa força interior, ela está em nós e quando confiarmos em nosso potencial, veremos como muito iremos realizar, como as mudanças virão e novos horizontes também. Acredite, a força interior pulsa em nós. Hoje e sempre..." 


\section{Agradecimentos}

Quero começar agradecendo a Deus, que através do meu espírito, me dá força e vontade para fazer tudo o que eu quero sem ter duvidar. Por isso, que eu posso hoje escrever estes agradecimentos que simboliza o final do meu trabalho e que ironicamente, se coloca no começo. Sou muito grato à vida (isto é, Deus) por ter me permitido percorrer esta grande viagem que me fortaleceu como amigo, como filho, como irmão, como pai, como homen, e evidentemente, como matemático.

Agradeço a minha familia toda, pois sempre estiveram nos momentos que eu precisei para me dar força, comprenssão e paz. Govinda, Paloma e filhos, por sua alegria inesgotável. À minha mãe por suas palavras sempre sábias. À meu pai por sua comprenssão. Aos meus irmãos Tania e Pablo, porque sempre estiveram aquí comigo. Aos meus papadres por seu apoio incondicional em todo momento.

Em especial, agradeço a uma mulher que faz parte do grande pilar que me firma, que é a grande luz que ilumina meu caminho e que me dá força nos momentos difíceis. Porque foi através do seu apoio que eu pude acabar com esta tarefa. Agradeço a esta mulher, que é a mãe do meus filhos, por confiar em mim (e portanto, nela). Por isso Maria Mónica, muito obrigado.

Aos meus filhos, alegria da minha vida, benção sagrada, motivação para seguir este caminho e nunca desistir, a eles agradeço por seu amor incondicional e me motivar a construir este trabalho.

Agradeço três grandes professores e pessoas que eu conheci:

- Primeiramente, o professor Jose Eduardo Prado Pires de Campos, meu orientador. Grande companheiro de trabalho que me ajudou em todo momento que foi preciso com minhas dúvidas matemáticas e existenciais. A ele agradeço por ter uma enorme paciência e comprenssão comigo, como matemático ou como pessoa, e também porque muito pude aprender com ele; 
- o segundo, o professor Oziride Manzonileto, pois ele solucionou muitas dúvidas e esteve disponivel, mesmo, nem sendo meu orientador, de me ajudar com algum problema matemático com total confiança, de me passar um jis para eu expor meu conflitos na louça que ele tem na sala dele;

- e o terceiro, mas não por isso menos importante, o professor Jesus Juyumaya, agradeço por ter aprensentado a teoria de nós e de como estes se representam por fechos de tranças, quando eu era apenas um graduando. Agora posso dizer que aquela matéria da graduação foi só uma introdução de uma pequena parte da teoria.

Agradeço também a todos os professores com os quais eu têve a oportunidade de conviver, pois com eles aprendi muito mais da matemática e me enriqueci matematicamente.

Aos meus amigos da USP, mestrandos e doutorandos do ICMC, pois com eles estudei e aprendi constantemente e porque sempre estiveram presentes para o que eu necessitasse.

Aos meus amigos do Alojamento, porque sempre alegraram os meus dias e diminuiram a saudade de casa.

Agradeço o importante apoio de CAPES-Coordenação de Aperfeiçoamento de Pessoal de Nível Superior, pela bolsa de mestrado que me permitiu estudar em ótimas condições, o qual provocou em mim um desempenho muito bom. Agradeço a FAFESP-Fundação de Amparo e Pesquisa do Estado de São Paulo, projeto tematico número: 08/57607-6. 


\section{Resumo}

A partir de um grupo $H$ e um elemento $h$ em $H$, nós definimos uma representação $\rho: B_{n} \rightarrow \operatorname{Aut}\left(H^{* n}\right)$, onde $B_{n}$ denota o grupo de trança de $n$ cordas, e $H^{* n}$ denota o produto livre de $n$ cópias de $H$. Chamamos a $\rho$ a representação de tipo Artin associada ao par $(H, h)$. Nós também estudamos varios aspectos de tal representação.

Primeiramente, associamos a cada trança $\beta$ um grupo $\Gamma_{(H, h)}(\beta)$ e provamos que o operador $\Gamma_{(H, h)}$ determina um grupo invariante de enlaçamentos orientados. Então damos uma construção topológica da representação de tipo Artin e do invariante de enlaçamentos $\Gamma_{(H, h)}$, e provamos que a representação é fiel se, e somente se, $h$ é não trivial. 


\section{Abstract}

From a group $H$ and a element $h \in H$, we define a representation $\rho: B_{n} \rightarrow \operatorname{Aut}\left(H^{* n}\right)$, where $B_{n}$ denotes the braid group on $n$ strands, and $H^{* n}$ denotes the free product of $n$ copies of $H$. We call $\rho$ the Artin type representation associated to the pair $(H, h)$. Here we study various aspects of such representations.

Firstly, we associate to each braid $\beta$ a group $\Gamma_{(H, h)}(\beta)$ and prove that the operator $\Gamma_{(H, h)}$ determines a group invariant of oriented links. We then give a topological construction of the Artin type representations and of the link invariant $\Gamma_{(H, h)}$, and we prove that the Artin type representations are faithful if and only if $h$ is nontrivial. 


\section{Sumário}

Introdução

1 Grupos Livres 3

1.1 Definição e Propriedades Básicas . . . . . . . . . . . . . . . . . . 3

1.2 Construção de Grupos Livres e suas Propriedades . . . . . . . . . . . . . . 5

1.3 Geradores e Relatores . . . . . . . . . . . . . . . . . . . . . . . 10

1.4 Produtos Livres . . . . . . . . . . . . . . . . . . . . . . . . 14

1.5 Push-Out e Extensões HNN . . . . . . . . . . . . . . . . . . 16

2 Grupóides $\quad 21$

2.1 Definição e Propriedades . . . . . . . . . . . . . . . . . . 21

2.2 Homomorfismos de Grupóides . . . . . . . . . . . . . . . . . 25

2.3 Extensões de Homomorfismos . . . . . . . . . . . . . . . . . . . 27

2.4 Push-Out de Grupóides . . . . . . . . . . . . . . . . . . . . . . . . . . 28

3 Elementos de Topologia e Topologia Algébrica 43

3.1 Topologia Fraca e Topologia Quociente . . . . . . . . . . . . . . . . . 43

3.2 Adjuntando Espaços mediante Funções . . . . . . . . . . . . . . . . . . 45

3.3 Caminhos e Homotopias . . . . . . . . . . . . . . . . . 46

3.4 O Grupóide Fundamental e o Grupo Fundamental . . . . . . . . . . . . . . 49

3.5 O Teorema de Seifert-Van Kampen . . . . . . . . . . . . . . . . 53

3.6 Complexo CW . . . . . . . . . . . . . . . . . . . 57

4 Tranças Geométricas de Artin e Enlaçamentos $\quad 59$

4.1 Tranças Geométricas . . . . . . . . . . . . . . . . . . . . . . 59

4.2 Representação de Tranças por Automorfismos de Grupos Livres . . . . . . 65 
4.3 Construindo Enlaçamentos a partir de Tranças . . . . . . . . . . . . . . . 68

4.4 Representando um Enlaçamento pelo Fecho de uma Trança . . . . . . . . . 70

4.5 Equivalência Combinatória de Tranças Fechadas . . . . . . . . . . . . . 72

4.5.1 Uma deformação do tipo $\mathscr{R}$. . . . . . . . . . . . . . . . . . . 72

4.5.2 Uma deformação do tipo $\mathscr{W} \ldots \ldots$. . . . . . . . . . . 72

4.6 O Grupo de um Enlaçamento . . . . . . . . . . . . . . . . . . 75

4.7 Algumas Definições da Teoria de Nós . . . . . . . . . . . . . . . . . . 77

5 A Representação do Tipo Artin do Grupo das Tranças $\quad 79$

5.1 Definição e Propriedades Elementares . . . . . . . . . . . . . . . 80

5.2 Enlaçamentos e Invariantes de Enlaçamentos . . . . . . . . . . . . . . . . 83

5.3 Construção Topológica do Invariante de Enlaçamentos . . . . . . . . . . . 85

5.4 Fidelidade da Representação de Tipo Artin . . . . . . . . . . . . . . . . . 100

5.5 Alguns Exemplos . . . . . . . . . . . . . . . . . 104 


\section{Introdução}

Em 1925, Artin introduziu o estudo de tranças, o qual se relaciona com o estudo de nós e enlaçamentos. Os resultados importantes obtidos por Artin foram seus teoremas da apresentação e da representação, que fornecem uma apresentação para o grupo das tranças no disco e de uma representação destas no grupo dos automorfimos (à direita) de um grupo livre em $n$ geradores.

A teoria de tranças se desenvolveu em várias direções com os trabalhos de Alexander, Markov, Wada e outros. A teoria básica pode ser encontrada em [2], e para uma rápida introdução pode se ver [3].

Neste trabalho, o principal objetivo é estudar uma representação do grupo de tranças no grupo dos automorfismos de um produto livre de $n$ cópias de um grupo arbitrário. Veremos que esta nova representação generaliza as representações dadas por Artin em 1925 e por Wada em 1992 (ver [8]). Provaremos que esta nova representação é fiel e, usando-a, construiremos um novo invariante de enlaçamentos.

Primeiramente, precisamos falar de grupos livres, conteúdo estudado no capítulo 1 (ver [1]). Na sequência, capítulo 2, estudamos grupóides e aplicamos este conteúdo no capítulo 3 para obter resultados importantes sobre grupos fundamentais. O capítulo 4 é uma introdução básica à teoria das tranças. E por último, no capítulo 5, expomos e demonstramos os principais resultados deste trabalho (ver [5]). 


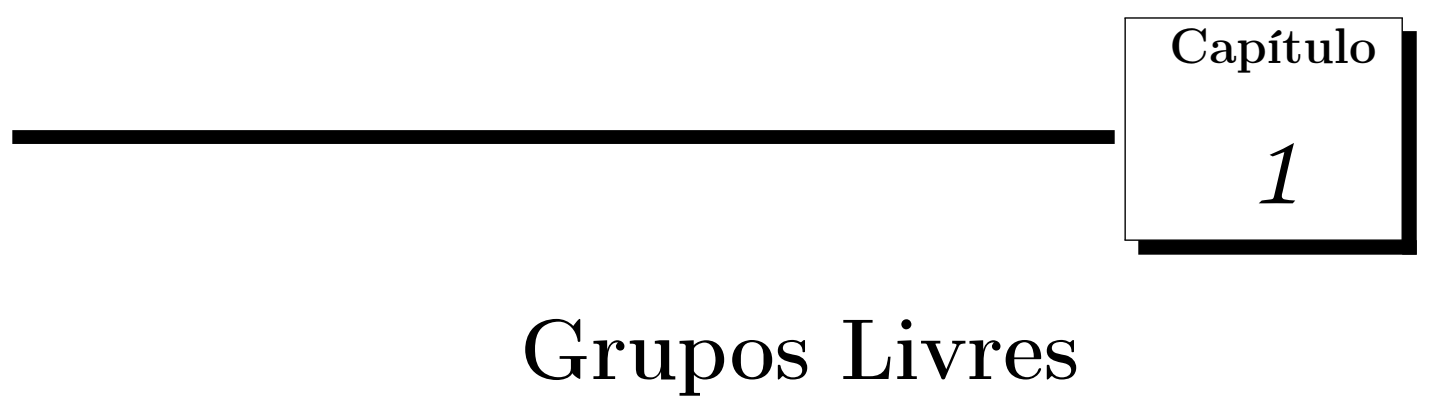

Em muitas aplicações da teoria de grupos e, especificamente, no cálculo de grupos fundamentais de espaços relacionados a enlaçamentos, os grupos são descritos por "relações definidoras", ou, como diremos mais tarde, encontrando uma "apresentação"dos grupos. O estudo das apresentações de grupos, deve começar com uma descrição do grupo livre para entender como se constróem estas apresentações.

\subsection{Definição e Propriedades Básicas}

Seja $G$ um grupo e $X$ um conjunto de geradores de $G$. Certamente, o produto de um elemento de $X$ com seu inverso sempre será $e_{G}$, o elemento neutro de $G$, qualquer que seja $X$ e $G$, por exemplo, $a b a a^{-1} b^{-1} a^{-1}=e_{G}$. Produtos de outro tipo, como $a b c$, ou $b a$, serão $e_{G}$, dependendo da escolha de $X$ e $G$. Vamos estudar aqueles pares $(G, X)$ onde o grupo $G$ satifaz só, e somente só, os axiomas básicos de grupos que todos os grupos satisfazem. Aqueles grupos serão chamados de grupos livres.

Seja $(G, X)$ um par com aquela propriedade. Considere um grupo $H$ e uma função $f: X \rightarrow H$. A função $f$ se estende a un único homomorfismo $\varphi: G \rightarrow H$. De fato, defina para $g=x_{i_{1}}^{\varepsilon_{1}} \ldots x_{i_{n}}^{\varepsilon_{n}}$, com $x_{i_{r}}$ em $X$ e $\varepsilon_{r}$ em $\{-1,1\}$, para $r=1, \ldots, n$,

$$
\varphi(g)=f\left(x_{i_{1}}\right)^{\varepsilon_{1}} \ldots f\left(x_{i_{n}}\right)^{\varepsilon_{n}}
$$

Afirmamos que $\varphi(g)$ não depende da forma como $g$ foi expresso. Suponhamos $g=x_{j_{1}}^{\delta_{1}} \ldots x_{i_{m}}^{\delta_{m}}$ 
$\operatorname{com} x_{i_{k}}$ em $X$ e $\delta_{k}$ em $\{-1,1\}$, para $k=1, \ldots, m$. Então

$$
x_{i_{1}}^{\varepsilon_{1}} \ldots x_{i_{n}}^{\varepsilon_{n}} x_{j_{m}}^{-\delta_{m}} \ldots x_{i_{1}}^{-\delta_{1}}=e_{G}
$$

Logo,

$$
\varphi\left(x_{i_{1}}^{\varepsilon_{1}} \ldots x_{i_{n}}^{\varepsilon_{n}} x_{j_{m}}^{-\delta_{m}} \ldots x_{i_{1}}^{-\delta_{1}}\right)=f\left(x_{i_{1}}\right)^{\varepsilon_{1}} \ldots f\left(x_{i_{n}}\right)^{\varepsilon_{n}} f\left(x_{j_{m}}\right)^{-\delta_{m}} \ldots f\left(x_{i_{1}}\right)^{-\delta_{1}}=e_{H}
$$

Assim, $\varphi(g)$ depende só de $g$ e não da forma como $g$ foi escrito. Portanto, $\varphi$ está bem definida. É claro que $\varphi$ é um homomorfismo e que restrita a $X$ é igual a $f$. Além disso, $\varphi$ está unicamente determinada pela escolha das imagens dos elementos de $X$. Neste caso, vamos dizer que $f$ se estende de forma natural para $G$.

Agora, suponha que $X$ é um subconjunto de um grupo $G$ tal que qualquer aplicação $f: X \rightarrow H$ se estende, de maneira única, a um homomorfismo de $G$ em $H$. Se $x_{i_{1}}^{\varepsilon_{1}} \ldots x_{i_{n}}^{\varepsilon_{n}}=$ $e_{G}$, como $f$ se estende a um homomorfismo de $G$ em $H, f\left(x_{i_{1}}\right)^{\varepsilon_{1}} \ldots f\left(x_{i_{n}}\right)^{\varepsilon_{n}}=e_{H}$. Da arbitrariedade do grupo $H$ e da aplicação $f$, isto equivale a dizer que o produto de elementos de $X \cup X^{-1}$ é $e_{G}$ só quando o produto associado através de $f$, no grupo correspondente, também é a identidade para todo grupo $H$ e aplicação $f$. Isto motiva a seguinte definição.

Definição 1.1.1. Seja $X$ um conjunto, $G$ um grupo e $i: X \rightarrow G$ uma aplicação. O par $(G, i)$ é chamado livre sobre $X$ se, para qualquer grupo $H$ e aplicação $f: X \rightarrow H$, existe um único homomorfismo $\varphi: G \rightarrow H$ tal que $f=\varphi \circ i$.

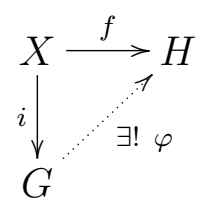

Por exemplo, o grupo trivial é livre sobre o conjunto vazio $\emptyset$, pois qualquer função do $\emptyset$ num grupo $H$, se estende ao homomorfismo trivial. O grupo aditivo $\mathbb{Z}$ é livre sobre um conjunto com um elemento $\{x\}$, onde a aplicação $i:\{x\} \rightarrow \mathbb{Z}$ é definida por $i(x)=1$, de fato, seja $f$ uma aplicação de $\{x\}$ em um grupo $H$ e escreva $f(x)=a$. Estendendo $f$ de maneira natural para $\mathbb{Z}$, obtemos $\varphi: \mathbb{Z} \rightarrow H$ definida por $\varphi(n)=a^{n}$. Este homomorfismo é único, pois se existir outro homomorfismo $\varphi^{\prime}$ de $\mathbb{Z}$ em $H$ com as propriedades de $\varphi$, então $f(x)=\varphi \circ i(x)=\varphi^{\prime} \circ i(x)$. Logo, $\varphi(1)=\varphi^{\prime}(1)=a$, como 1 é gerador de $\mathbb{Z}$ temos que $\varphi=\varphi^{\prime}$ sobre $\mathbb{Z}$, e portanto, $\varphi$ é único. 
Proposição 1.1.2. Seja $\left(G_{1}, i_{1}\right)$ livre sobre $X$. Então, outro par $\left(G_{2}, i_{2}\right)$ é livre sobre $X$, se e somente se, existe um isomorfimo $\phi: G_{1} \rightarrow G_{2}$ tal que $\phi \circ i_{1}=i_{2}$.

Demonstração. Primeiro suponha que existe um isomorfimo $\phi \operatorname{de} G_{1}$ em $G_{2}$ tal que $\phi \circ i_{1}=$ $i_{2}$. Seja $H$ um grupo e $f: X \rightarrow H$ uma aplicação qualquer. Então, existe um único homomorfismo $\varphi: G \rightarrow H$ tal que $f=\varphi \circ i_{1}$. É claro que o homomorfismo $\psi: G_{2} \rightarrow H$ definido por $\psi=\varphi \circ \phi^{-1}$ é único e satisfaz $f=\psi \circ i_{2}$. Portanto, $\left(G_{2}, i_{2}\right)$ é livre sobre $X$.

Reciprocamente, suponha que $\left(G_{2}, i_{2}\right)$ é livre sobre $X$. Pela definição de grupo livre sobre $X$, existem dois homomorfismos únicos, $\phi: G_{1} \rightarrow G_{2}$ e $\psi: G_{2} \rightarrow G_{1}$ tais que $i_{2}=\phi \circ i_{1}$ e $i_{1}=\psi \circ i_{2}(\operatorname{ver}(1.1 .4))$. Logo, $\phi \circ \psi \circ i_{2}=i_{2}$ e $\psi \circ \phi \circ i_{1}=i_{1}$. Por outro lado, é claro que $i_{1}=I d_{G_{1}} \circ i_{1}$ e $i_{2}=I d_{G_{2}} \circ i_{2}$. Da unicidade na definição de grupo livre temos $\psi \circ \varphi=I d_{G_{1}}$ e $\phi \circ \psi=I d_{G_{2}}$. Portanto, $\phi$ é um isomorfismo que satisfaz $i_{2}=\phi \circ i_{1}$.

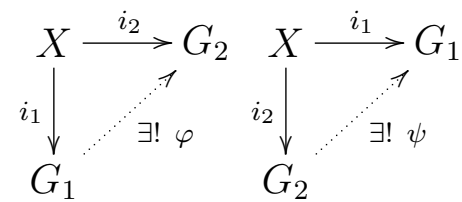

Proposição 1.1.3. Seja $(F, i)$ um grupo livre sobre um conjunto $X$, então $i$ é injetora.

\subsection{Construção de Grupos Livres e suas Propriedades}

Seja $X$ um conjunto. Defina $M(X)$ como o conjunto que contém todas as sequências finitas $\left(x_{i_{1}}, \ldots, x_{i_{n}}\right)$ de elementos de $X$, com $n \geq 0$. No caso $n=0$, a sequência é vazia e escrevemos (). Defina uma multiplicação em $M(X)$ por

$$
\left(x_{i_{1}}, \ldots, x_{i_{n}}\right) \cdot\left(x_{j_{1}}, \ldots, x_{j_{m}}\right)=\left(x_{i_{1}}, \ldots, x_{i_{n}}, x_{j_{1}}, \ldots, x_{j_{m}}\right)
$$

Esta multiplicação é associativa e tem elemento neutro, a saber, a palavra vazia, que denotaremos por 1. Assim, $(M(X), \cdot)$ tem estrutura de monóide ${ }^{1}$, e será chamado de monóide livre em $X$.

\footnotetext{
${ }^{1}$ Um monóide é um conjunto $G$ com uma operação de $G \times G$ em $G$, associativa e com elemento neutro. A diferença entre um monóide e um grupo, é que no monóide nem todo elemento precisa ter inverso.
} 
Observação 1.2.1. A aplicação $x \longmapsto\{x\}$ é injetora, portanto, identificando $x$ com $(x)$ temos que todo elemento do monóide livre em $X$ é produto de elementos de $X$.

Observação 1.2.2. É possivel que o conjunto $X$ tenha algum elemento que já é uma sequência de outros elementos de $X$, e nós queremos diferenciar entre os elementos de $X$ e $M(X)$. Para solucionar isto, identifica-se $X$ com o conjuto $X^{\prime}$, formado pelos elementos da forma $\{x\}$, com $x$ em $X$. Logo, definimos o monóide livre de $X$ sendo $M\left(X^{\prime}\right)$. Ignoraremos este detalhe no futuro.

\section{Construção:}

Vamos construir um grupo livre sobre um conjunto arbitrário $X$. Considere um conjunto $\bar{X}$ em bijeção com $X$ por meio da aplicação $x \longmapsto \bar{x}$, e tal que $X \cap \bar{X}=\emptyset$. Em geral, os elementos de $\bar{X}$ são denotados por $x^{-1}$, e o símbolo $x^{1}$ corresponde a $x$. Os elementos de $M(X \cup \bar{X})$ serão chamados palavras sobre $X$, e denotaremos por $w u$ o produto $w \cdot u$ em $M(X \cup \bar{X})$. Se $w$ é uma palavra $x_{i_{1}}^{\varepsilon_{1}} \ldots x_{i_{n}}^{\varepsilon_{n}}$ sobre $X$, entenderemos por comprimento de $w$, denotado por $l(w)$ ou $|w|$, o número de elementos de $X \cup \bar{X}$ que aparecem na escrita de $w$, isto é, $l(w)=n$. Os elementos $x_{i_{r}}^{\varepsilon_{r}} \in X$ são chamados letras de $w$.

Seja $w$ a palavra $x_{i_{1}}^{\varepsilon_{1}} \ldots x_{i_{n}}^{\varepsilon_{n}}$ sobre $X$. Diremos que $w$ é reduzida, se ela é a palavra vazia ou, se para cada $r=1, \ldots, n-1, i_{r} \neq i_{r+1}$ ou $i_{r}=i_{r+1}$ mas $\varepsilon_{r} \neq-\varepsilon_{r+1}$. Suponha que $w$ não seja reduzida e escolha um $r$ tal que $i_{r+1}=i_{r}$ e $\varepsilon_{r+1}=-\varepsilon_{r}$. Seja $w^{\prime}$ a palavra obtida de $w$ eliminando o produto $x_{i_{r}}^{\varepsilon_{r}} x_{i_{r+1}}^{\varepsilon_{r+1}}$ na escrita de $w$. Dizemos que $w^{\prime}$ é obtida de $w$ por redução elementar. Se $w^{\prime \prime}$ é outra palavra sobre $X$, obtida por uma sequência de reduções elementares de $w$, dizemos que $w^{\prime \prime}$ é obtida de $w$ por redução.

Exemplo 1.2.3. Seja w a palavra $a b c^{-1} c b^{-1} b a$. As palavras abb ${ }^{-1} b a$ e $a b c^{-1} c a$, são obtidas de w por redução elementar, e a palavra aba é obtida de w por redução.

Dadas duas palavras $w$ e $w^{\prime}$ sobre $\mathrm{X}$, escrevemos $w \approx w^{\prime}$ quando $w$ for identica a $w^{\prime}$ ou, se existe uma sequência finita de palavras $w_{1}, \ldots, w_{k}$, para algum inteiro positivo $k$ tal que $w_{1}$ é $w, w_{k}$ é $w^{\prime}$ e, para cada $1 \leq j<k, w_{j+1}$ é obtido de $w_{j}$ por redução elementar, ou vice-versa. É fácil ver que $\approx$ é uma relação de equivalência sobre $M(X \cup \bar{X})$. Denotemos por $F(X)$ o conjunto das classes de equivalência de $M(X \cup \bar{X})$ sob $\approx$ e denotemos por $[w]$, a classe da palavra $w$. Definamos um produto em $F(X)$ por $[u] \cdot[w]=[u w]$. As seguintes observações mostram que o produto $\cdot$ em $F(X)$ está bem definido. 
Observação 1.2.4. Sejam $u, v, w, w^{\prime}$ palavras em $X$.

- Se $w \approx w^{\prime}$ então $u w v \approx u w^{\prime} v$. De fato, sem perda de generalidade, suponhamos que $w_{1}, \ldots, w_{k}$ é uma sequência de palavras sobre $X$ com $w_{1}=w, w_{k}=w^{\prime}$ e $w_{j+1}$ obtida por redução elementar de $w_{j}$, ou viceversa, para todo $1 \leq j<k$. Logo, a sequência $u w_{1} v, \ldots, u w_{k} v$, mostra que uwv $\approx u w^{\prime} v$.

- Se $u \approx u^{\prime}$ e $w \approx w^{\prime}$ então $u w \approx u^{\prime} w^{\prime}$. De fato, ambas são equivalentes a uw'.

Assim, o produto $\cdot$ em $F(X)$ está bem definido.

Proposição 1.2.5. $(F(X), \cdot)$ é um grupo.

Demonstração. A propriedade associativa é clara. O elemento neutro em $F(X)$ é a classe da palavra vazia, denotada por 1 . Dada a classe da palavra $x_{i_{1}}^{\varepsilon_{1}} \ldots x_{i_{r}}^{\varepsilon_{r}}$ em $F(X)$, consideremos a classe da palavra $x_{i_{r}}^{-\varepsilon_{r}} \ldots x_{i_{1}}^{-\varepsilon_{1}}$, É claro que o produto dessas classes é 1 e, portanto, cada classe en $F(X)$ tem inverso.

Seja $w=x_{i_{1}}^{\varepsilon_{1}} \ldots x_{i_{r}}^{\varepsilon_{r}}$ uma palavra sobre $X$ e definamos $i: X \rightarrow F(X)$ por $i(x)=[x]$. Como $[w]=\left[x_{i_{1}}^{\varepsilon_{1}}\right] \ldots\left[x_{i_{r}}^{\varepsilon_{r}}\right]$, segue que, $[w]=i\left(x_{i_{1}}^{\varepsilon_{1}}\right) \ldots i\left(x_{i_{r}}^{\varepsilon_{r}}\right)$. Portanto, a imagem de $X$ sob $i$ gera $F(X)$, ou seja, todo elemento de $F(X)$ é um produto de elementos de $i(X)$. Com estas notações, temos o seguinte teorema.

Teorema 1.2.6. $(F(X), i)$ é livre sobre $X$.

Demonstração. Seja $G$ um grupo e $f: X \rightarrow G$ uma aplicação arbitrária. Estenda $f$ para $\bar{f}: M(X \cup \bar{X}) \rightarrow G$ por

$$
\bar{f}\left(x_{i_{1}}^{\epsilon_{1}} \ldots x_{i_{n}}^{\epsilon_{n}}\right)=f\left(x_{i_{1}}\right)^{\epsilon_{1}} \ldots f\left(x_{i_{n}}\right)^{\epsilon_{n}}
$$

Segue que, se $w^{\prime}$ é uma palavra sobre $X$ obtida por redução elementar de outra palavra $w$, então $\bar{f}\left(w^{\prime}\right)=\bar{f}\left(w^{-1}\right)$, assim, se $w^{\prime \prime}$ é um palavra sobre $X$ obtida por redução de $w$, então $\bar{f}\left(w^{\prime \prime}\right)=\bar{f}(w)$.

Defina $\varphi: F(X) \rightarrow G$ por $\varphi([w])=\bar{f}(w)$. Pelo que vimos acima, $\varphi$ está bem definida. Além disso, $\varphi$ é um homomorfismo pela definição de $\bar{f}$, e claramente, $\varphi \circ i=f$. Como $i(X)$ gera $F(X), \varphi$ está determinada de forma única. Segue que $(F(X), i)$ é livre sobre $X$. 
Como as reduções elementares decrescem o comprimento de cada palavra, é natural pensar que através de reduções elementares acharemos una palavra reduzida, mas, é única? Isso é o que responde o teorema da forma normal para grupos livres apresentado a seguir, o qual não iremos demonstrar.

Teorema 1.2.7 (Forma Normal para Grupos Livres). Existe uma, e somente uma palavra reduzida em cada classe de equivalência de $F(X)$.

O teorema acima fornece outra prova do seguinte resultado.

Corolário 1.2.8. A aplicação $i: X \rightarrow F(X)$ definida por $i(x)=[x]$, é injetora.

Geralmente,

- Vamos considerar o conjunto $X$ como um subconjunto de $F(X)$ e a função $i$ como a inclusão, consequentemente, trocaremos $i(x)$ por simplesmente $x$.

- Vamos escolher como representante de cada classe em $F(X)$ a palavra reduzida associada em $M(X \cup \bar{X})$.

- Vamos diferenciar explicitamente quando estejamos trabalhando com elementos de $M(X \cup \bar{X})$ ou $F(X)$, como segue.

Notação: Denotaremos por

- $w \equiv w^{\prime}$, quando $w$ e $w^{\prime}$ sejam palavras identicas em $M(X \cup \bar{X})$.

- $w=w^{\prime}$, quando $w$ e $w^{\prime}$ definam o mesmo elemento em $F(X)$, isto é, $w$ e $w^{\prime}$ sejam equivalentes.

Observação 1.2.9. Sejam u e v palavras reduzidas, então existe somente uma sequência de reduções elementares que podem ser aplicadas a uv para obter uma palavra reduzida.

Demonstração. De fato, seja $u \equiv x_{i_{1}}^{\varepsilon_{1}} \ldots x_{i_{n}}^{\varepsilon_{n}}$ e $v \equiv x_{j_{1}}^{\delta_{1}} \ldots x_{i_{m}}^{\delta_{m}}$. Tome $s \geq 0$ o número total de termos tais que para $0 \leq r \leq s$ temos

$$
i_{n+1-r}=j_{r} \text { e } \varepsilon_{n+1-r}=-\delta_{r}
$$


Para $r=1, \ldots, s$, da única forma possv́el, elimine os termos $x_{n+1-r}^{\varepsilon_{n+1-r}} x_{r}^{\delta_{r}}$ em $u v$, obtendo a palavra

$$
x_{i_{1}}^{\varepsilon_{1}} \ldots x_{i_{n-s}}^{\varepsilon_{n-s}} x_{j_{s}}^{\delta_{s}} \ldots x_{j_{m}}^{\delta_{m}}
$$

a qual é reduzida.

Observação 1.2.10. É possivel definir $F(X)$ como o conjunto das palavras reduzidas, $e$ o produto $\cdot$ de duas palavras u e $v$ como a palavra reduzida obtida de uv.

Proposição 1.2.11. $F(X)$ é isomorfo a $F(Y)$ se, e somente se, $|X|=|Y|$

Definição 1.2.12. Um grupo $G$ é chamado livre, se é isomorfo a $F(X)$ para algum conjunto $X$. Se $i: F(X) \rightarrow G$ é um isomorfismo, então chamamos ao conjunto $i(X)$ base de $G$ e dizemos que $G$ é livre em $i(X)$.

Segue da proposição 1.2.11 e da definição 1.2 .12 a seguinte observação.

\section{Observação 1.2.13.}

(i) Se A é uma base de $G$ e a é um automorfismo de $G$, então $\alpha(A)$ também é uma base de $G$.

(ii) Se $A$ e $B$ são bases de um mesmo grupo, então $|A|=|B|$.

(iii) Toda bijeção entre as bases $A$ e $B$ de $G$, se estende de forma natural para um automorfismo de $G$.

A observação 1.2.13 permite dar a seguinte definição.

Definição 1.2.14. A cardinalidade de uma base de um grupo livre $G$ é chamada o posto de $G$

Proposição 1.2.15. Seja $X$ um subconjunto de um grupo G. São equivalentes:

(1) G é um grupo livre com base $X$

(2) Todo elemento de $G$ se escreve de maneira única como $x_{i_{1}}^{\varepsilon_{1}}, \ldots, x_{i_{n}}^{\varepsilon_{n}}$, para algum $n \geq 0, x_{i_{r}}$ em $X$ e $\varepsilon_{r}= \pm 1$, onde $\varepsilon_{r+1} \neq-\varepsilon_{r}$ se $i_{r+1}=i_{r}$. 
(3) $X$ gera $G$ e e $e_{G}$ não é igual a qualquer produto $x_{i_{1}}^{\varepsilon_{1}}, \ldots, x_{i_{n}}^{\varepsilon_{n}}$, com $n>0, x_{i_{r}}$ em $X$ e $\varepsilon_{r}= \pm 1$, onde $\varepsilon_{r+1} \neq-\varepsilon_{r}$ se $i_{r+1}=i_{r}$.

Corolário 1.2.16. Seja $X$ conjunto gerador de $G$ e seja $\varphi: G \rightarrow H$ um homomorfismo de grupos que é um a um em $G$ e tal que $\varphi(G)$ é livre com base $\varphi(X)$. Então, G é livre com base $X$.

Corolário 1.2.17. Seja $G$ livre com base $X$ e seja $Y$ um subconjunto de $X$. Então o subgrupo de $G$ gerado por $Y,\langle Y\rangle$, é livre com base $Y$.

Corolário 1.2.18. Seja $F$ livre com base $\{x, y\}$. Seja $\varphi: F \rightarrow \mathbb{Z}$ o homomorfismo definido por $\varphi(x)=1$ e $\varphi(y)=0$. Então, $\operatorname{ker}(\varphi)$ é livre com base $A=\left\{x^{-i} y x^{i}: i \in \mathbb{Z}\right\}$.

Proposição 1.2.19. Grupos livres são livres de torsão, isto é, todo elemento de G tem ordem infinita.

Proposição 1.2.20. Seja F um grupo livre e $g$ e $h$ elementos em F. Se gh=hg então $\langle g, h\rangle$ é cíclico, isto é, existe um elemento $u$ em $F$ e inteiros $r$ e s tais que $g=u^{r} e$ $h=u^{s}$.

\subsection{Geradores e Relatores}

Proposição 1.3.1. Todo grupo G é quociente de algum grupo livre.

Demonstração. Considere a aplicação identidade de $G, I d_{G}: G \rightarrow G$. Esta aplicação se estende a outra aplicação $\varphi: F(G) \rightarrow G$ tal que $I d_{G}=\varphi \circ i$, onde $i: G \rightarrow F(G)$ é definida por $i(g)=[g]$.

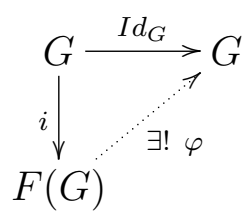

Portanto, $\varphi$ é sobrejetora. Segue o resultado do teorema do homomorfismo. 
Definição 1.3.2. Seja $G$ um grupo, $X$ um conjunto e $\varphi: F(X) \rightarrow G$ um epimorfismo. Então:

(1) X é chamado conjunto de símbolos geradores $(\operatorname{sob} \varphi)$ para $G$.

(2) A familia $\varphi(X)=\{\varphi(x): x \in X\}$ é chamado um conjunto de geradores de $G$.

(3) $\operatorname{ker}(\varphi)$ é chamado conjunto de relatores de $G(s o b \varphi)$.

(4) Dadas duas palavras quaisquer $u \equiv x_{i_{1}}^{\varepsilon_{1}} \ldots x_{i_{n}}^{\varepsilon_{n}}$ e $v \equiv x_{j_{1}}^{\delta_{1}} \ldots x_{j_{m}}^{\delta_{m}}$ tais que uv ${ }^{-1}$ está no kernel de $\varphi$, pondo $a_{i}=\varphi\left(x_{i}\right)$, dizemos que $a_{i_{1}}^{\varepsilon_{1}} \ldots a_{i_{n}}^{\varepsilon_{n}}=a_{j_{1}}^{\delta_{1}} \ldots a_{j_{m}}^{\delta_{m}}$ é uma relação $\mathrm{em}$ $G$.

Definição 1.3.3. Para todo subconjunto $S$ de um grupo $H$, o fecho normal $\langle S\rangle^{H}$ em $H$ do subgrupo $\langle S\rangle$ é chamado o conjunto das consequências de $S$ em $H$, ou simplesmente, o subgrupo normal de $H$ gerado por $S$.

Definição 1.3.4. Seja $G$ um grupo, $X$ um conjunto e $\varphi: F(X) \rightarrow G$ um epimorfismo. Se $\operatorname{ker}(\varphi)$ é o conjunto das consequências de algum subconjunto $R$ de $F(X)$ (isto é, $\operatorname{ker}(\varphi)=$ $\left.\langle R\rangle^{F(X)}\right)$, então chamamos a $R$ um conjunto de relatores definidores de $G(\operatorname{sob} \varphi)$. Diremos que uma relação $u=v$ em $G$ é uma consequência de relatores definidores se $u v^{-1}$ (relator associado) é uma consequência de relatores definidores.

Definição 1.3.5. Uma apresentação $\langle X ; R\rangle^{\varphi}$ de $G$, consiste de um conjunto $X$, um epimorfismo $\varphi: F(X) \rightarrow G$ e um conjunto $R$ de relatores definidores de $G$ sob $\varphi$. Dizemos que o grupo $G$ é apresentado por $\langle X ; R\rangle^{\varphi}$ e escrevemos $G=\langle X ; R\rangle^{\varphi}$.

As vezes omitiremos na escrita colocar $\varphi$, em especial quando $\varphi$ for a aplicação natural de $F(X)$ em $F(X) /\langle R\rangle^{F(X)}$ ou quando $\varphi$ for injetora sobre $X$. Quando $X$ e $R$ forem finitos, diremos que $\langle X ; R\rangle^{\varphi}$ é uma apresentação finita de $G$ e que $G$ é um grupo finitamente apresentado.

\section{Exemplo 1.3.6.}

(1) $F(X)$ tem apresentação $\langle X ; R\rangle^{\varphi}$ com $R$ vazio. Neste caso, $\varphi$ é a função identidade de $F(X)$. 
(2) A apresentação $\left\langle x, y ; x y^{2}=y^{3} x, y x^{2}=x^{3} y\right\rangle$ apresenta o grupo trivial.

Demonstração. Observe que

$$
x y^{4} x^{-1}=\left(x y^{2} x^{-1}\right)^{2}=y^{6}
$$

então

$$
x^{2} y^{4} x^{-2}=x y^{6} x^{-1}=\left(x y^{2} x^{-1}\right)^{3}=y^{9}
$$

Segue que

$$
y^{9}=x^{2} y^{4} x^{-2}=y x^{2} y^{4} x^{-2} y^{-1}=y x^{2} y^{-1} y^{4} y x^{-2} y^{-1}=x^{3} y^{4} x^{-3}
$$

portanto, $x^{2} y^{4} x^{-2}=x^{3} y^{4} x^{-3}$. Logo $y^{4}=x y^{4} x^{-1}=y^{6}$ e assim $y^{2}=1$. Segue que $y=x=1$.

Teorema 1.3.7 (Von Dyck). Seja $G=\langle X ; R\rangle^{\varphi}$ e $f: X \rightarrow H$ uma função em algum grupo H. Seja $\xi: F(X) \rightarrow H$ o homomorfismo associado a $f$. Se $\xi(r)=1$ para todo $r$ em $R$, então existe um homomorfismo $\psi: G \rightarrow H$ tal que $f(x)=\psi \circ \varphi(x)$ para todo $x$ em $X$. Além disso, se $f(X)$ gera $H$ então $\psi$ é um epimorfismo.

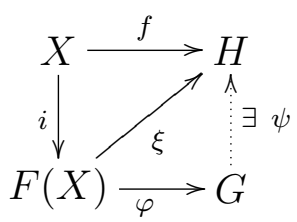

Corolário 1.3.8. A inclusão $i: X \rightarrow X \cup Y$ induz um homomorfismo de $\langle X ; R\rangle^{\varphi}$ em $\langle X \cup Y ; R \cup S\rangle^{\psi}$ para todo subconjunto $S$ de $F(X \cup Y)$.

A seguinte proposição é um caso particular do teorema do homomorfimo, e é possível prová-la usando o teorema de Von-Dyck.

Proposição 1.3.9. Seja $R$ um subconjunto de um grupo $A$ e seja $\xi: A \rightarrow H$ um homomorfismo de grupos com $\xi(R)=\{1\}$, então existe um homomorfismo $\psi: A /\langle R\rangle^{A} \rightarrow H$ tal que $\xi=\psi \circ \pi$ onde $\pi: A \rightarrow A /\langle R\rangle^{A}$ é o homomorfismo natural. 
É possível que um grupo tenha várias apresentações, as seguintes definições e o posterior teorema esclarecem esta questão.

Seja $G$ um grupo apresentado por $\langle X ; R\rangle^{\varphi}$. Para $S \subset\langle R\rangle^{F(X)}=\operatorname{ker}(\varphi)$, a apresentação $\langle X ; R \cup S\rangle^{\varphi}$ também é uma apresentação de $G$, pois $\langle R \cup S\rangle^{F(X)}=\operatorname{ker}(\varphi)$. Diremos então que a apresentação $\langle X ; R \cup S\rangle^{\varphi}$ provém de $\langle X ; R\rangle^{\varphi}$ por uma transformação geral de Tietze do tipo I, e que $\langle X ; R\rangle^{\varphi}$ provém de $\langle X ; R \cup S\rangle^{\varphi}$ por uma transformação geral de Tietze do tipo I'. Quando $|S|=1$, nos referimos a transformações simples de Tietze.

Seja $Y$ um conjunto disjunto de $X$. Escolha para cada elemento $y$ de $Y$, um elemento $u_{y}$ em $F(X)$. Defina $\psi: F(X \cup Y) \rightarrow G$ por $\psi(x)=\varphi(x)$ para $x$ em $X$ e $\psi(y)=\varphi\left(u_{y}\right)$ para $y$ em $Y$. Então $\left\langle X \cup Y ; R \cup\left\{y u_{y}^{-1}: y \in Y\right\}\right\rangle^{\psi}$ também apresenta $G$. De fato, seja $N=\left\langle R \cup\left\{y u_{y}^{-1}: y \in Y\right\}\right\rangle^{F(X \cup Y)}, \psi$ induz um epimorfismo $\pi: F(X \cup Y) / N \rightarrow G$ definido por $w N \longmapsto \varphi(w)$, pois $\psi\left(R \cup\left\{y u_{y}^{-1}: y \in Y\right\}\right)=\{1\}$. Do teorema de Von-Dyck, existe um epimorfismo $\xi: G \rightarrow F(X \cup Y) / N$ tal que $\xi \circ \varphi(x)=x N$ para todo $x$ em $X$. Claramente $\pi \circ \xi$ é a identidade. Observe que dado $y$ em $Y$,

$$
\xi \circ \pi(y N)=\xi \circ \psi(y)=\xi \circ \varphi\left(u_{y}\right)=u_{y} N=y N
$$

Portanto $\xi \circ \pi$ é o homomorfismo identidade de $F(X \cup Y) / N$. Segue o resultado. Dizemos então que $\left\langle X \cup Y ; R \cup\left\{y u_{y}^{-1}: y \in Y\right\}\right\rangle^{\psi}$ provém de $\langle X ; R\rangle^{\varphi}$ por uma transformação geral de Tietze do tipo II e que $\langle X ; R\rangle^{\psi}$ provém de $\left\langle X \cup Y ; R \cup\left\{y u_{y}^{-1}: y \in Y\right\}\right\rangle^{\psi}$ por uma transformação geral de Tietze do tipo II'. Quando $|Y|=1$ nos referimos a transformações simples.

Teorema 1.3.10. Duas apresentações quaisquer de um mesmo grupo podem ser obtidas uma da outra mediante uma sequência de transformaçôes gerais de Tietze. Se ambas as apresentações são finitas então cada uma pode ser obtida da outra por uma sequência de transformações simples de Tietze.

Proposição 1.3.11. Seja $G$ finitamente gerado e $\langle Y ; S\rangle^{\psi}$ uma apresentação de G. Então existe um subconjunto finito de $Y$ que gera $G$ (sob $\psi)$.

Proposição 1.3.12. Sejam $\langle X ; R\rangle^{\varphi}$ e $\langle Y ; S\rangle^{\psi}$ duas apresentações de um mesmo grupo G. Se $X, R$ e $Y$ são finitos então existe $S_{1} \subset S$ tal que $G$ é apresentado por $\left\langle Y ; S_{1}\right\rangle^{\psi}$ 


\subsection{Produtos Livres}

Nesta seção vamos generalizar o conceito de grupo livre e suas propriedades.

Definição 1.4.1. Seja $\left\{G_{\alpha}\right\}$ uma familia de grupos, $G$ um grupo e para cada $\alpha$, seja $i_{\alpha}$ : $G_{\alpha} \rightarrow G$ um homomorfismo. $\left(G,\left\{i_{\alpha}\right\}\right)$ é chamado produto livre da família $\left\{G_{\alpha}\right\}$ se para todo grupo $H$ e família de homomorfismos $f_{\alpha}: G_{\alpha} \rightarrow H$ existe um único homomorfismo $f: G \rightarrow H$ tal que $f_{\alpha}=f \circ i_{\alpha}$, para todo $\alpha$.

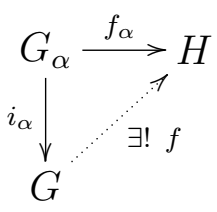

Proposição 1.4.2. Se $\left(G,\left\{i_{\alpha}\right\}\right)$ e $\left(H,\left\{j_{\alpha}\right\}\right)$ são ambos produtos livres de uma familia de grupos $\left\{G_{\alpha}\right\}$, então existe um único isomorfismo $f: G \rightarrow H$ tal que $f \circ i_{\alpha}=j_{\alpha}$, para todo $\alpha$.

Teorema 1.4.3. Toda familia de grupos $\left\{G_{\alpha}\right\}$ tem um produto livre.

Demonstração. Suponhamos que para cada $\alpha, G_{\alpha}$ é apresentado por $\left\langle X_{\alpha}: R_{\alpha}\right\rangle^{\varphi_{\alpha}}$. Assuma, sem perda de generalidade, que $X_{\alpha} \cap X_{\beta}=\emptyset$, para todo $\alpha \neq \beta$. Seja

$$
G=F\left(\cup_{\alpha} X_{\alpha}\right) /\left\langle\cup_{\alpha} R_{\alpha}\right\rangle^{F\left(\cup_{\alpha} X_{\alpha}\right)}
$$

$G$ é apresentado por $\left\langle\cup_{\alpha} X_{\alpha}: \cup_{\alpha} R_{\alpha}\right\rangle^{\varphi}$, onde $\varphi: F\left(\cup_{\alpha} X_{\alpha}\right) \rightarrow G$ é a aplicação natural. Pelo corolário do teorema de Von Dyck, para cada $\alpha$, a inclusão $\widetilde{i_{\alpha}}: X_{\alpha} \rightarrow \underset{\alpha}{\cup} X_{\alpha}$ induz um homomorfismo $i_{\alpha}: G_{\alpha} \rightarrow G$. Vamos provar que $\left(G,\left\{i_{\alpha}\right\}\right)$ é o produto livre da familia de grupos $\left\{G_{\alpha}\right\}$.

Seja $H$ um grupo e para cada $\alpha$, seja $f_{\alpha}: G_{\alpha} \rightarrow H$ um homomorfismo. Queremos encontrar um homomorfismo $f: G \rightarrow H$ tal que $f \circ i_{\alpha}=f_{\alpha}$. De fato, $f_{\alpha}$ induz um homomorfismo

$$
\psi_{\alpha}=f_{\alpha} \circ \varphi_{\alpha}: F\left(X_{\alpha}\right) \rightarrow H
$$


tal que $\psi_{\alpha}\left(R_{\alpha}\right)=\{1\}$, para todo $\alpha$. Defina o homomorfimo

$$
\psi: F\left(\cup_{\alpha} X_{\alpha}\right) \rightarrow H
$$

por $\psi(x)=\psi_{\alpha}(x)$, para todo $x \in X_{\alpha}$. É claro que $\psi\left(\underset{\alpha}{\cup} R_{\alpha}\right)=\{1\}$, portanto, do teorema de Von Dyck, existe $f: G \rightarrow H$ tal que $\psi=f \circ \varphi$.

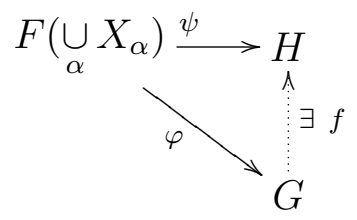

Observe que para cada $\alpha,\left(i_{\alpha} \circ \varphi_{\alpha}\right)\left(x_{\alpha}\right)=\varphi\left(x_{\alpha}\right)$, para todo $x_{\alpha} \in X_{\alpha}$.

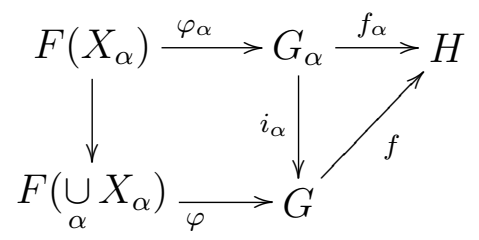

Portanto,

$$
\begin{aligned}
f\left(i_{\alpha}\left(\varphi_{\alpha}\left(x_{\alpha}\right)\right)\right) & =f\left(\varphi\left(x_{\alpha}\right)\right) \\
& =\psi\left(x_{\alpha}\right) \\
& =\psi_{\alpha}\left(x_{\alpha}\right) \\
& =f_{\alpha}\left(\varphi_{\alpha}\left(x_{\alpha}\right)\right), \forall x_{\alpha} \in X_{\alpha}
\end{aligned}
$$

Como $\varphi_{\alpha}\left(X_{\alpha}\right)$ gera $G_{\alpha}$, temos $f \circ i_{\alpha}=f_{\alpha}$, para todo $\alpha$. Além disso, $f$ é única pois $\cup_{\alpha}\left(i_{\alpha} \circ \varphi_{\alpha}\right)\left(X_{\alpha}\right)$ gera $G$.

Quando $G$ é o produto livre de uma família $\left\{G_{\alpha}\right\}_{\alpha}$, escrevemos $G=*_{\alpha} G_{\alpha}$. Quando o conjunto que percorre $\alpha$ seja $\{1, \ldots, n\}$, escrevemos $G=G_{1} * \cdots * G_{n}$.

Exemplo 1.4.4. O grupo livre $F(X)$ é o produto livre da familia de grupos cíclicos

$$
\{\langle x\rangle: x \in X\}
$$


Teorema 1.4.5 (Forma Normal). Seja $\left(G,\left\{i_{\alpha}\right\}\right)$ o produto livre de uma família de grupos $\left\{G_{\alpha}\right\}$. Então,

(1) Para cada $\alpha, i_{\alpha}$ é um monomorfismo.

(2) Considerando $i_{\alpha}$ como a inclusão, cada elemento não trivial de $G$ é escrito de maneira única como $g_{1} \ldots g_{n}$, com $n \geq 0, g_{i} \in G_{\alpha_{i}}$ para algum $\alpha_{i}, g_{i} \neq 1$ para todo $i=1, \ldots$, n e $\alpha_{r} \neq \alpha_{r+1}$ para todo $r<n$.

Proposição 1.4.6. Seja $\left\{G_{\alpha}\right\}$ uma família de subgrupos de um grupo G. São equivalentes:

(i) G é o produto livre da família $\left\{G_{\alpha}\right\}$.

(ii) Cada elemento de $G$ é escrito de maneira única como um produto $g_{1} \ldots g_{n}$, com $n \geq 0, g_{i} \in G_{\alpha_{i}}, g_{i} \neq 1$, e $\alpha_{i} \neq \alpha_{i+1}$.

(iii) $G$ é gerado pelos subgrupos $G_{\alpha}$ e 1 não pode ser escrito como um produto $g_{1} \ldots g_{n}$, com $n>0, g_{i} \in G_{\alpha_{i}}, g_{i} \neq 1$, e $\alpha_{i} \neq \alpha_{i+1}$.

\subsection{Push-Out e Extensões HNN}

Definição 1.5.1. Sejam $G_{0}, G_{1}, G_{2}$ grupos e sejam $i_{1}: G_{0} \rightarrow G_{1}$ e $i_{2}: G_{0} \rightarrow G_{2}$ homomorfismos. Seja $G$ um grupo, e sejam $j_{1}: G_{1} \rightarrow G, j_{2}: G_{2} \rightarrow G$ homomorfismos. Dizemos que $\left(G, j_{1}, j_{2}\right)$ é o push-out de $\left(i_{1}, i_{2}\right)$ se as seguintes condições se verificam:

(i) $j_{1} \circ i_{1}=j_{2} \circ i_{2}$

(ii) Para cada grupo $H$ e homomorfismos $\varphi_{r}: G_{r} \rightarrow H, r=1,2$, com $\varphi_{1} \circ i_{1}=\varphi_{2} \circ i_{2}$, existe um único homomorfismo $\varphi: G \rightarrow H$ tal que $\varphi_{r}=\varphi \circ j_{r}, r=1,2$.

Assim, dizemos que temos um quadrado (ou diagrama) push-out:

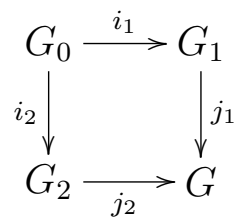


Usualmente faremos referência ao push-out mencionando apenas o grupo $G$ em vez do trio $\left(G, j_{1}, j_{2}\right)$.

Proposição 1.5.2. O push-out $G$ na definição, é único a menos de isomorfismo.

Demonstração. Seja $\left(L, l_{1}, l_{2}\right)$ outro push-out de $\left(i_{1}, i_{2}\right)$. Pela definição anterior, existe um único homomorfismo $\alpha: G \rightarrow L$ tal que $\alpha \circ j_{1}=l_{1}$ e $\alpha \circ j_{2}=l_{2}$, e por outro lado, existe um único homomorfismo $\beta: L \rightarrow G$ tal que $\beta \circ l_{1}=j_{1}$ e $\beta \circ l_{2}=j_{2}$. Logo, $\alpha \circ \beta \circ l_{i}=l_{i}$, para $i=1,2$. Como $I d_{L}$ também satifaz esta propriedade, temos $I d_{L}=\alpha \circ \beta$. Analogamente $I d_{G}=\beta \circ \alpha$. Segue o resultado.

Teorema 1.5.3. Todo par $\left(i_{1}, i_{2}\right)$ como na definição 1.5.1, tem um push-out.

Demonstração. Suponhamos que o grupo $G_{r}$ seja apresentado por $\left\langle X_{r} ; R_{r}\right\rangle^{\xi_{r}}$, para $r=$ 1,2 , com $X_{1} \cap X_{2}=\emptyset$. Seja $Y$ um conjunto gerador de $G_{0}$ e escolhamos para cada $y$ em $Y$ um elemento $w_{y r}$ em $F\left(X_{r}\right)$ tal que

$$
i_{r}(y)=\xi_{r}\left(w_{y r}\right), \text { para } r=1,2 .
$$

Seja $G$ o grupo apresentado por $\left\langle X_{1} \cup X_{2} ; R_{1}, R_{2},\left\{w_{y 1}^{-1} w_{y 2}\right\}\right\rangle$. Assim, para $r=1,2$, temos aplicações naturais $j_{r}: G_{r} \rightarrow G$, induzidas pelas inclusões de $X_{r}$ em $X_{1} \cup X_{2}$ e portanto $G$ é gerado por $j_{1}\left(G_{1}\right) \cup j_{2}\left(G_{2}\right)$. Desta forma, pode existir no máximo um homomorfismo saindo de $G$ com valores especificados em $j_{1}\left(G_{1}\right) \cup j_{2}\left(G_{2}\right)$.

Para $r=1,2$, seja $\varphi_{r}: G_{r} \rightarrow H$ um homomorfismo em um grupo $H$ tal que $\varphi_{1} \circ i_{1}=$ $\varphi_{2} \circ i_{2}$. Logo, para $r=1,2, \varphi_{r}$ define um homomorfismo $\psi_{r}: F\left(X_{r}\right) \rightarrow H$ que é trivial em $R_{r}$. Pelo teorema de Von-Dick, o homomorfismo de domínio $F\left(X_{1} \cup X_{2}\right)$ que é $\psi_{r}$ em $X_{r}$ define um homomorfismo $\varphi: G \rightarrow H$ que satisfaz $\varphi_{r}=\varphi \circ j_{r}$, para $r=1,2$. Segue que $G$ é o push-out procurado.

Observação 1.5.4. O teorema 1.5.3 fornece uma apresentação para um push-out de grupos.

Exemplo 1.5.5. Se $G_{2}$ é trivial então o push-out é o grupo quociente $G_{1} /\left\langle i_{1}\left(G_{0}\right)\right\rangle^{G_{1}}$. 
Quando $i_{1}$ e $i_{2}$ forem homomorfismos injetores, chamamos ao push-out $G$ produto livre amalgamado de $G_{1}$ e $G_{2} \operatorname{com} G_{0}$ amalgamado. Neste caso, geralmente consideramos $G_{0}$ como um subgrupo de $G_{1}$ e $G_{2}$ e $i_{1}, i_{2}$ como inclusões. A notação usual para esta situação é $G_{1_{G_{0}}^{*}} G_{2}$. Às vezes, por conveniência, usa-se a seguinte notação: $G_{1_{G_{0}}=H_{0}} G_{2}$ onde $G_{0} \subseteq G_{1}, H_{0} \subseteq G_{2}, i_{1}$ é a inclusão e $i_{2}$ é um isomorfismo entre $G_{0}$ e $H_{0}$. Para maior precisão, pode-se mencionar o isomorfismo específico de $G_{0}$ em $H_{0}$.

Em 1949 Graham Higman, B. H. Neumann e Hanna Neumann, no artigo intitulado "Embedding Theorems for Groups", introduziram uma construção ralacionada a produtos livres amalgamados, a qual mais tarde foi chamada Extensão HNN. Vamos apresentar uma construção padrão de uma extensão HNN em vez de usar a propriedade universal de sua definição original.

Sejam $G$ e $A$ grupos. Sejam $i_{0}$ e $i_{1}$ monomorfismos de $A$ em $G$ e seja $P$ um grupo cíclico infinito com gerador $p$ que não é um gerador de $G$. Consideremos o grupo normal

$$
N=\left\langle p^{-1} i_{o}(a) p i_{1}(a)^{-1}: a \text { é gerador de } A\right\rangle^{G * P}
$$

O grupo $(G * P) / N$ é chamado extensão HNN do grupo base $G$ com letra estável $p$ e subgrupos associados $i_{0}(A)$ e $i_{1}(A)$. Usualmente consideraremos $A$ um subgrupo de $G$ e $i_{0}$ como sendo a inclusão e escreveremos

$$
(G * P) / N=\left\langle G, p ; p^{-1} A p=i_{1}(A)\right\rangle
$$

Observação 1.5.6. Suponhamos que $G$ seja apresentado por $\langle X ; R\rangle^{\varphi} \operatorname{com} p \notin X$. Seja $Y$ um subconjunto de $F(X)$ e seja $\xi: Y \rightarrow F(X)$ uma aplicação injetora. Seja $A=\langle\varphi(Y)\rangle$ e $B=\langle\varphi \circ \xi(Y)\rangle$. Se $\xi$ induz um isomorfismo de $A$ em $B$ então a extensão HNN tem apresentação

$$
\left\langle X, p ; R, p^{-1} y p=\xi(y), y \text { em } Y\right\rangle
$$

É possível estender o conceito de extensão HNN para uma família de grupos $\left\{A_{\alpha}\right\}$ e de monomorfismos $\left\{i_{0 \alpha}\right\}$ e $\left\{i_{1 \alpha}\right\}$ de $A_{\alpha}$ em $G$. Seja $P$ o grupo livre sobre um conjunto $\left\{p_{\alpha}\right\}$. Neste caso, a extensão HNN é

$$
H=G * P /\left\langle p_{\alpha}^{-1} i_{0 \alpha}\left(a_{\alpha}\right) p_{\alpha}=i_{1 \alpha}\left(a_{\alpha}\right): a_{\alpha} \text { gerador de } A_{\alpha}, \forall \alpha\right\rangle^{G * P}
$$

Se considerarmos a mesma construção acima mas com os homomorfismos $i_{0 \alpha}$ e $i_{1 \alpha}$ não 
sendo monomorfismos, falaremos de uma pseudo extensão HNN.

\section{Exemplo 1.5.7.}

1. O grupo livre com base $\left\{p_{\alpha}\right\}$ é uma extensão $H N N$ do grupo trivial com letras estáveis $p_{\alpha}$.

2. Seja A um subgrupo de um grupo G. É possivel formar a extensão $H N N$

$$
\left\langle G, k ; k^{-1} a k=a, \text { a gerador de } A\right\rangle
$$

3. O grupo apresentado por $\left\langle a, b ; a^{-1} b a=b^{2}\right\rangle$ é a extensão $H N N$ do grupo cíclico $\langle b\rangle$ com letra estável a e subgrupos associados $\langle b\rangle$ e $\left\langle b^{2}\right\rangle$.

Proposição 1.5.8. Seja $H$ a extensão $H N N$ de $G$ com letras estáveis $\left\{p_{\alpha}\right\}$ e com subgrupos associados $i_{0 \alpha}\left(A_{\alpha}\right)$ e $i_{1 \alpha}\left(A_{\alpha}\right)$. O homomorfismo inclusão de $G$ em $G * P$ induz um homomorfismo $j: G \rightarrow H$, o qual é um monomorfismo.

A propriedade universal das extensões HNN é dada na seguinte proposição.

Proposição 1.5.9. Sejam $G$ e $K$ grupos e seja $\varphi$ um homomorfismo de $G$ em $K$. Suponhamos que $K$ tem elementos $k_{\alpha}$ tais que

$$
k_{\alpha}^{-1} \varphi\left(i_{0 \alpha}\left(a_{\alpha}\right)\right) k_{\alpha}=\varphi\left(i_{1 \alpha}\left(a_{\alpha}\right)\right)
$$

para todo $\alpha$ e para todo $a_{\alpha} \in A_{\alpha}$. Seja $H$ a extensão $H N N$ de $G$ com letras estáveis $\left\{p_{\alpha}\right\}$ e subgrupos associados $i_{0 \alpha}\left(A_{\alpha}\right)$ e $i_{1 \alpha}\left(A_{\alpha}\right)$. Então existe um único homomorfismo $\psi: H \rightarrow K$ tal que $\psi \circ j=\varphi$ e $\psi\left(p_{\alpha}\right)=k_{\alpha}$, para todo $\alpha$. 


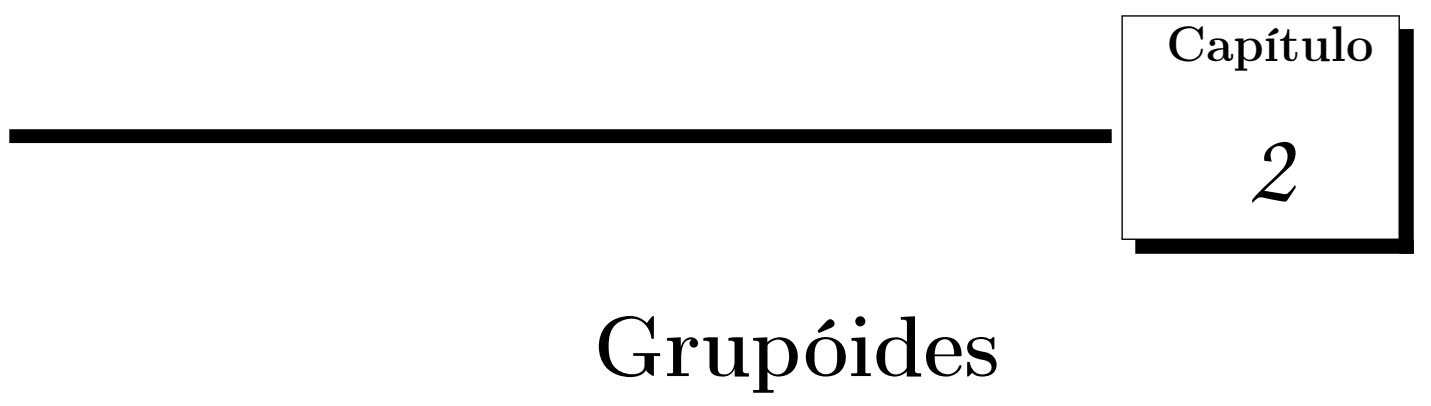

Neste capítulo vamos estudar as propriedades de grupóides que serão de utilidade no estudo de um invariante de enlaçamentos no capítulo 5. Em particular, nosso interesse está no estudo de três tipos diferentes de push-out de grupóides que são aplicados para obter corólarios do teorema de Van-Kampen e assim, calcular grupos fundamentais de certos espaços topológicos.

\subsection{Definição e Propriedades}

Definição 2.1.1. Uma multiplicação parcial sobre um conjunto $G$ é uma função de algum subconjunto $X$ de $G \times G$ em $G$. Denotamos a imagen de $(x, y)$ em $X$ sob esta multiplicação parcial por $x \cdot y$, ou simplesmente $x y$. Dizemos que xy está definido quando $(x, y)$ está em $X$.

Definição 2.1.2. Um elemento e em $G$ é uma identidade de $G$ com respeito a uma multiplicação parcial $\cdot$, se ex $=x$ e ye $=y$ sempre que ex e ye estão definidos.

Definição 2.1.3. Um grupóide $G$ é um conjunto com uma multiplicação parcial tal que

(1) (Associatividade) Se um dos elementos $(a b)$ c ou a(bc) está definido, então o outro também está definido e são iguais. Além disso, se ab e bc estão definidos então $(a b) c$ (e a $(b c)$ ) está definido. 
(2) (Existência de identidades) Para todo a em $G$ existem identidades e e $f$ em $G$, tais que ea e af são definidos.

(3) (Existência de inverso) Para todo a em $G$ e, e e f como em (2), existe um elemento em $G$ que denotaremos por $a^{-1}$ tal que $a a^{-1}=e$ e $a^{-1} a=f$.

Observação 2.1.4. Se e e $f$ são identidades tais que ef está definido então $e=f$.

Demonstração. Isto é claro pois pela definição de identidade $f=e f=e$.

\section{Exemplo 2.1.5.}

(i) Todo grupo é um grupóide.

(ii) Seja $\left\{X_{\lambda}\right\}_{\lambda \in \Lambda}$ uma familia de grupos. Para cada $\lambda$ e $\mu$ em $\Lambda$, consideremos o conjunto

$$
G_{\lambda \mu}=\left\{f: X_{\lambda} \rightarrow X_{\mu} / f \text { é uma bijeção }\right\}
$$

Então $G=\cup_{\lambda, \mu \in \Lambda} G_{\lambda \mu}$ é um grupóide sob a composição de funções.

As propriedades mais simples de grupóides são muito similares áquelas de grupos, com algumas modificações necessarias, já que a multiplicação em um grupóide $G$ nem sempre está definida.

Observação 2.1.6. Para cada elemento a em $G$ pode existir só uma identidade e tal que ea está definido. Analogamente, pode existir só uma identidade f tal que af está definido.

Demonstração. Suponhamos que $e_{1}$ é outra identidade tal que $e_{1} a$ está definido. Logo, $e\left(e_{1} a\right)$ está definido pois $e_{1} a=a$, assim, pela propriedade associativa, $e e_{1}$ está definido. O resultado segue da observação 2.1.4. O outro caso é analogo.

Observação 2.1.7. O inverso $a^{-1}$ de um elemento a em $G$ é unico.

Demonstração. Suponhamos que $a^{\prime}$ é outro inverso de $a$ tal que $a^{\prime} a=e$ e $a a^{\prime}=f$, onde $e$ e $f$ são identidades de $a$. Suponhamos também que $a^{-1} a=e$ e $a a^{-1}=f$, então

$$
a^{\prime}=a^{\prime} f=a^{\prime}\left(a a^{-1}\right)=\left(a^{\prime} a\right) a^{-1}=e a^{-1}=a^{-1}
$$


Observação 2.1.8. A equação $a x=b$ tem solução somente se $a^{-1} b$ está definido. Neste caso, $a^{-1} b$ é a única solução.

Demonstração. Suponhamos que $a^{-1} b$ está definido. Logo, $a\left(a^{-1} b\right)$ está definido e é igual a $\left(a a^{-1}\right) b=e b=b$, pois $a a^{-1}$ está definido e igual a $e$. Logo, $a^{-1} b$ é solução de $a x=b$. Reciprocamente, suponha que existe um $x$ em $G$ tal que $a x=b$. Como $a^{-1} a$ está definido e é igual a uma identidade $f$, segue que $\left(a^{-1} a\right) x=f x$ está definido e é portanto igual a $x$. Assim, $a^{-1}(a x)$ está definido e é igual a $x$, mas $a x=b, \operatorname{logo} a^{-1} b=x$.

Observação 2.1.9. A equação ya $=b$ tem solução se, e somente se, ba ${ }^{-1}$ está definido. Neste caso, a solução é única e é igual a ba $a^{-1}$.

Demonstração. É analoga à anterior.

Observação 2.1.10. Todo elemento e tal que ee =e é uma identidade.

Demonstração. Existe uma identidade $f$ para o elemento $e$ tal que ef está definido e $e f=e$. Segue que $e e=e f=e$. Como a equação $e x=e$ tem solução única segue que $e=f$, que é uma identidade.

Observação 2.1.11. ab está definido se, e somente se, existe uma identidade $f$ tal que af e fb estão definidos.

Demonstração. Se $a f$ e $f b$ estão definidos então $a(f b)$ está definido e é igual a $a b$. Reciprocamente, se $a b$ está definido, considere a identidade $f$ tal que af está definido, então $(a f) b$ está definido e portanto $a(f b)$ está definido. Assim, $f b$ está definido.

Observação 2.1.12. Se um grupóide $G$ tem só uma identidade, então para todo a e b em $G$, ab está sempre definido, e portanto, $G$ é um grupo.

Definição 2.1.13. Chamaremos as identidades de um grupóide $G$ de vértices de G. Seja a em $G$ e e e $f$ identidades tais que ea e af estão definidos. Dizemos que a é uma seta de e a $f$, e que e é o vértice inicial (ou, o começo) de a, e que f é o vértice final (ou simplesmente, final) de a. Denotamos o conjunto dos vértices do grupóide $G$ por $V(G)$.

Definição 2.1.14. Seja $V$ um conjunto qualquer. 
(1) $D(V)$ será o grupóide cujo conjunto de vértices é $V$ e tal que para cada $v$ e w em $V$ distintos, existe uma única seta de $v$ a $v$, mas não existe seta de $v$ a $w$.

(2) $T(V)$ será o grupóide com conjunto de vértices igual a $V$ e tal que para cada $v$ e w em $V$ existe uma única seta de $v$ a $w$.

A multiplicação parcial definida sobre estes conjuntos é a justaposição de setas. $D(V)$ é chamado grupóide discreto sobre $V$ e $T(V)$ é chamado o grupóide árvore sobre $V$.

Definição 2.1.15. Um subgrupóide $H$ de um grupóide $G$ é um subconjunto de $G$ tal que

(1) Se a e b estão em $H$ com ab definido, então ab está em $H$.

(2) Se $a \in H$, então $a^{-1} \in H$.

Observação 2.1.16. Seja $H$ subgrupóide de $G$. Se a está em $H$ e e e f são identidades tais que ea e af estão definidos, então e e f estão em $H$.

Demonstração. Como $H$ é subgrupóide de $G$, então $a^{-1}$ está em $H$. Portanto, $a a^{-1}$ e $a^{-1} a$ estão em $H$. Assim $e$ e $f$ estão em $H$.

\section{Exemplo 2.1.17.}

(i) Seja $G$ um grupo, então todo subgrupo de $G$ é também um subgrupóide.

(ii) A interseção de qualquer coleção de subgrupóides de um grupóide é um subgrupóide.

(iii) Para um conjunto $V$, o grupóide discreto $D(V)$ é um subgrupóide do grupóide árvore $T(V)$.

Definição 2.1.18. Seja $G$ um grupóide e $S$ um subconjunto de $V(G)$. Definamos $G_{S}$ como sendo o conjunto das setas de $G$ que começam e acabam em $S$. Então $G_{S}$ é um subgrupóide chamado subgrupóide completo de $G$ com conjunto de vértices $S$. Em particular, se $S=\{e\}$ escrevemos $G_{e}$ em vez de $G_{\{e\}}$. Neste caso, $G_{e}$ tem só uma identidade e portanto, $G_{e} e$ um grupo (observação 2.1.12), que chamaremos grupo vértice de $G$ em e. 
Observação 2.1.19. O conjunto $G_{S}$, como na definição 2.1.18, é de fato um subgrupóide de $G$.

Demonstração. Claramente $G_{S}$ é um subconjunto de $G$. Sejam $a$ e $b$ setas em $G_{S}$ com $a b$ definido. Então $a b$ é uma seta que começa e acaba em $S$, portanto $a b$ está em $G_{S}$. Além disso, $a^{-1}$ também é uma seta que começa e acaba em $S$.

Seja $G$ um grupóide e e e $f$ em $V(G)$. Definimos uma relacão $\equiv$ em $V(G)$ por

$$
e \equiv f \Leftrightarrow \text { existe uma seta em } G \text { de } e \text { a } f
$$

Evidentemente, $\equiv$ é uma relação de equivalência em $V(G)$. Vamos chamar as classes de equivalência de $V(G)$ sob $\equiv$ componentes de $G$ e denotaremos por $[v]$ a classe do vértice $v$ em $G$.

Definição 2.1.20. Seja G um grupóide.

(1) $G$ é dito conexo se existe uma única componente de $G$.

(2) G é chamado totalmente disconexo se cada componente consiste de um único vértice.

Exemplo 2.1.21. Sendo $V$ um conjunto não vazio, $T(V)$ é conexo e $D(V)$ é totalmente disconexo

\subsection{Homomorfismos de Grupóides}

Considere um grupóide $G$ e uma seta $a$ em $G$ começando em $e$ e acabando em $f$. Defina $a_{\#}: G_{e} \rightarrow G_{f}$ por $a_{\#}(g)=a^{-1} g a$. $a_{\#}$ é um isomorfismo entre os grupos $G_{e}$ e $G_{f}$ que satisfaz:

(1) Se $b$ é outra seta em $G$ começando em $f$ e acabando em algum otro vértice de $G$, então $(a b)_{\#}=b_{\#} \circ a_{\#}$.

(2) Se $e$ é uma identidade de $G$, então $e_{\#}$ é o homomorfismo identidade de $G_{e}$.

(3) $\left(a_{\#}\right)^{-1}=\left(a^{-1}\right)_{\#}$ é o homomorfismo inverso de $a_{\#}$. 
Note que se $c$ é uma seta em $G_{e}$, então $c_{\#}$ é a conjugação por $c$ no grupo $G_{e}$. Segue que se $b$ é uma seta em $G$ de $e$ a $f$, então $a_{\#}$ e $b_{\#}$ são homomorfismos conjugados. Isto é claro, pois para $g$ em $G_{e}$

$$
\begin{aligned}
a_{\#}(g) & =a^{-1} g a \\
& =a^{-1} b b^{-1} g b b^{-1} a \\
& =\left(b^{-1} a\right)^{-1} b_{\#}(g)\left(b^{-1} a\right)
\end{aligned}
$$

Como $a_{\#}$ é um isomorfismo de grupos, segue a seguinte proposição.

Proposição 2.2.1. Se e e $f$ são vértices numa mesma componente, então $G_{e}$ e $G_{f}$ são isomorfos. Em particular, os grupos vértice de um grupóide conexo são isomorfos.

Definição 2.2.2. Sejam $G$ e $H$ grupóides e $\varphi: G \rightarrow H$ uma função. Dizemos que $\varphi$ é um homomorfismo de grupoides (ou simplesmente, homomorfismo) se sempre que $a b$ estiver definido então $\varphi(a) \varphi(b)$ está definido e vale $\varphi(a) \varphi(b)=\varphi(a b)$.

Observação 2.2.3. Seja $\varphi: G \rightarrow H$ um homomorfismo de grupóides. Se e é uma identidade em $G$ então $\varphi(e)$ é uma identidade em $H$. Em particular, $\varphi$ induz uma funcão de $V(G)$ em $V(H)$ que também será denotada por $\varphi$.

Demonstração. Seja e uma identidade de $G$, logo, existe uma seta $a$ em $G$ tal que $e a=a$, e então, existe uma identidade $f$ tal que $a f=a$. Logo,

$$
e e=a a^{-1} a a^{-1}=a f a^{-1}=a a^{-1}=e
$$

Portanto, ee está definido. Como $\varphi$ é um homomorfismo, segue que $\varphi(e) \varphi(e)$ está definido e é igual a $\varphi(e)$. Da observação 2.1.10, $\varphi(e)$ é uma identidade.

Observação 2.2.4. Se a é uma seta de e a $f$ então $\varphi(a)$ é uma seta de $\varphi(e)$ a $\varphi(f)$ e $\varphi\left(a^{-1}\right)=\varphi(a)^{-1}$.

Demonstração. Temos que $e a=a$ e $a f=a$. Segue que $\varphi(e) \varphi(a)=\varphi(a)$ e $\varphi(a) \varphi(f)=$ $\varphi(a)$. Como $\varphi(e)$ e $\varphi(f)$ são identidades segue que $\varphi(a)$ é uma seta de $\varphi(e)$ a $\varphi(f)$.

Como $a a^{-1}=e, a^{-1} a=f$ e $\varphi$ é um homomorfismo, segue que $\varphi(a) \varphi\left(a^{-1}\right)=\varphi(e) \mathrm{e}$ $\varphi\left(a^{-1}\right) \varphi(a)=\varphi(f)$. Como o inverso é único, $\varphi\left(a^{-1}\right)=\varphi(a)^{-1}$. 


\subsection{Extensões de Homomorfismos}

Agora passamos a estudar extensões de funções para homomorfismos de grupóides. Aprenderemos a estender homomorfismos de grupos para homomorfismos de grupóides. Faremos uma aplicação disto no estudo de push-out de grupóides (seção 2.4).

Observação 2.3.1. Se $V$ e $W$ são conjuntos, então toda função de $V$ a $W$ induz homomorfismos de $D(V)$ em $D(W)$, e de $T(V)$ em $T(W)$.

Demonstração. Para uma seta $a$ de $v$ a $v$ em $D(V)$, escolha como imagem de $a$ a única seta $a^{\prime}$ em $D(W)$ de $f(v)$ a $f(v)$. Para uma seta $a$ de $v$ a $w$ em $T(V)$, escolha como imagen de $a$ a única seta $a^{\prime}$ em $T(W)$ de $f(v)$ a $f(w)$. É fácil ver que isto define os homomorfismos procurados.

Proposição 2.3.2. Seja $G$ um grupóide e seja $W$ um conjunto. Então, toda função $\varphi: V(G) \rightarrow W$ estende-se, de maneira única, a um homomorfismo $\phi: G \rightarrow T(W)$.

Demonstração. Dados os vértices e e $f$ em $G$, então $\varphi(e)$ e $\varphi(f)$ são vértices de $T(W)$. Portanto, para cada seta $g$ em $G$ de $e$ a $f$, defina $\phi(g)$ como sendo a única seta em $T(W)$ de $\varphi(e)$ a $\varphi(f)$. Afirmamos que $\phi$ assim definida é um homomorfismo de grupóides. De fato, sejam $a$ e $b$ setas em $G$ tal que $a b$ é definido. Existem vértices $e, f$ e $d$ tais que $e a$, $a f, f b$ e $b d$ estão definidos (pela observação 2.1.11). Logo, $\phi(a)$ é a única seta em $T(W)$ de $\varphi(e)$ a $\varphi(f)$ e $\phi(b)$ é única seta em $T(W)$ de $\varphi(f)$ a $\varphi(d)$. Logo, $\phi(a) \phi(f)$ e $\phi(f) \phi(b)$ estão definidos. Como $\varphi(f)$ é um vértice, segue que $\phi(a) \phi(b)$ está definido e além disso, $\phi(a) \phi(b)=\phi(a b)$ pois $\phi(a) \phi(b)$ é uma seta em $T(W)$ de $\varphi(e)$ a $\varphi(d)$.

A demonstração da seguinte proposição é análoga à anterior.

Proposição 2.3.3. Seja $G$ um grupóide e seja $W$ um conjunto. Toda aplicação $\psi: W \rightarrow$ $V(G)$ se estende, de maneira única, a um homomorfismo $\Psi: D(W) \rightarrow G$.

Proposição 2.3.4. Sejam $G$ e $H$ grupóides e seja $S$ um subconjunto de $V(G)$. Então todo homomorfismo $\psi: G_{S} \rightarrow H$ se estende (de maneira não necessariamente única) a um homomorfismo $\Psi: G \rightarrow H$. 
Demonstração. Para cada vértice $u$ de $G$ tal que $[u] \cap S \neq \emptyset$ escolha uma seta fixa $x_{u}$ de algum ponto de $S$ a $u$, e se $u$ está em $S$ escolha $x_{u}$ sendo uma identidade.

Seja $g$ uma seta em $G$ de um vértice $v$ a um vértice $w$ (caso exista). Se $[v]$ intercepta $S$ então $[w]$ também intercepta $S$ e portanto, $x_{v} g$ e $g x_{w}^{-1}$ estão definidos e assim $x_{v} g x_{w}^{-1}$ também está definido e está em $G_{S}$. Defina então $\Psi(g)=\psi\left(x_{v} g x_{w}^{-1}\right)$. Escolha $h$ em $V(H)$ fixo, se $[v] \cap S=\emptyset$, defina $\Psi(g)=h$. Isto implica $\Psi(v)=\Psi(w)=h$. Evidentemente, $\Psi$ estende $\psi$ pois dada uma seta $g$ em $G_{S}$ de $e$ a $f$, então $x_{e}=e$ e $x_{f}=f$. Assim, $\Psi(g)=\psi(g)$.

Para mostrar que $\Psi$ é um homomorfismo suponha que $a$ e $b$ são setas em $G$ tais que $a b$ está definido. Então, existem vértices $e, f$ e $d$ tais que $e a, a f, f b$ e $b d$ estão definidos. Suponha que $[e]$ intercepta $S$, logo, $x_{e} a x_{f}^{-1}$ e $x_{f} b x_{d}^{-1}$ estão em $G_{S}$, e portanto, $\psi\left(x_{e} a x_{f}^{-1}\right) \psi\left(x_{f} b x_{d}^{-1}\right)$ está definido pois $\psi$ é um homomorfismo. Segue que $\Psi(a) \Psi(b)$ está definido e é igual a $\Psi(a b)$. O caso em que $[e]$ não intercepta $S$ é trivial. Portanto, $\Psi$ é um homomorfismo.

Observação 2.3.5. Na proposição anterior, a extensão do homomorfismo $\psi$ não é única pois podem existir varias setas começando em $S$ chegando em algum vértice $v$ de $G$, aliás, uma vez feita a escolha das setas $x_{v}$ e de $h$ em $V(H)$, a extensão $\Psi$ fica unicamente determinada.

\subsection{Push-Out de Grupóides}

Definição 2.4.1. Sejam $G_{0}, G_{1}$ e $G_{2}$ grupóides e $i_{1}: G_{0} \rightarrow G_{1}$ e $i_{2}: G_{0} \rightarrow G_{2}$ homomorfismos. Suponha que existe um grupóide $G$ e homomorfismos $j_{1}: G_{1} \rightarrow G$ e $j_{2}: G_{2} \rightarrow G$. $O$ trio $\left(G, j_{1}, j_{2}\right)$ é chamado push-out de $\left(i_{1}, i_{2}\right)$ se as seguintes condições se verificam:

(1) $j_{1} \circ i_{1}=j_{2} \circ i_{2}$

(2) Para qualquer grupóide $H$ e homomorfismos $\varphi_{1}: G_{1} \rightarrow H$ e $\varphi_{2}: G_{2} \rightarrow H$ tais que $\varphi_{1} \circ i_{1}=\varphi_{2} \circ i_{2}$, existe um único homomorfismo $\varphi: G \rightarrow H$ tal que $\varphi_{1}=\varphi \circ j_{1} e$ $\varphi_{2}=\varphi \circ j_{2}$. 
Note que, no caso de um push-out, o diagrama abaixo comuta:

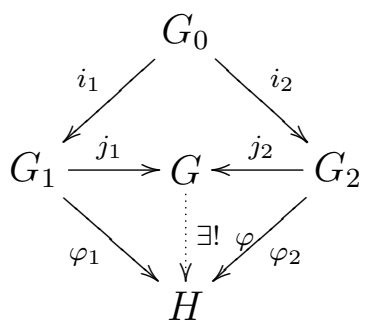

O diagrama (2.4.1) é chamado diagrama push-out de grupóides.

Observação 2.4.2. O trio $\left(G, j_{1}, j_{2}\right)$ é único a menos de isomorfismo.

A demonstração da observação 2.4.2 é igual à demonstração da observação 1.5.2.

Observação 2.4.3. Um push-out de grupóides no qual os grupóides são grupos, é um push-out de grupos. Por outro lado, todo push-out de grupos é um push-out de grupóides.

Por conveniência, dado um grupóide $N$ com pelo menos dois vértices $a$ e $b$, denotaremos por $N_{a b}$ o conjunto das setas em $N$ de $a$ a $b$.

Observação 2.4.4. Seja $N$ um grupóide conexo com $V(N)=\{a, b\}$. Dada uma seta $u$ em $N$ de a a b fixa, temos

$$
\begin{aligned}
& N_{a b}=\left\{n u: n \in N_{a}\right\} \\
& N_{b a}=\left\{u^{-1} n: n \in N_{a}\right\} \\
& N_{b}=\left\{u^{-1} n u: n \in N_{a}\right\}
\end{aligned}
$$

Demonstração. Basta observar que

(i) Se $n$ está em $N_{a b}$ então $n=n u^{-1} u$.

(ii) Se $n$ está em $N_{b a}$ então $n=u^{-1} u n$.

(iii) Se $n$ está em $N_{b}$ então $n=u^{-1} u n u^{-1} u$.

Definição 2.4.5. Nas condições da observação 2.4.4, diremos que $N_{a b}, N_{b a}$ e $N_{b}$ são definidos em função de $N_{a}$ e da seta $u$. 
Os push-out de importância para nós têm a propriedade adicional

$$
V\left(G_{0}\right)=V\left(G_{1}\right) \cap V\left(G_{2}\right) \text { e } V(G)=V\left(G_{1}\right) \cup V\left(G_{2}\right)
$$

e os homomorfismos serão as extensões das inclusões destes conjuntos nos outros. Pushout's com esta propriedade são chamados push-out's ponteados.

A seguinte proposição é usada frequentemente nos exemplos posteriores.

Proposição 2.4.6. Seja $N$ um grupóide conexo com $V(N)=\{a, b\}$. Seja $M$ outro grupóide conexo e $\psi: N_{a} \rightarrow M_{\psi(a)}$ um homomorfismo de grupos. Seja u uma seta em $N$ de a a b. Se $x$ for uma seta em $M$ de $\psi(a)$ a um vértice y de $M$, então

$$
\Psi(n)= \begin{cases}\psi(n) & , \text { se } n \in N_{a} \\ x & , \text { se } n=u\end{cases}
$$

define um homomorfismo $\Psi: N \rightarrow M$ que é único.

Demonstração. Para $n$ em $N_{a}$ defina

$$
\begin{gathered}
\Psi(n u)=\psi(n) x \\
\Psi\left(u^{-1} n\right)=x^{-1} \psi(n) \\
\Psi\left(u^{-1} n u\right)=x^{-1} \psi(n) x
\end{gathered}
$$

Demonstraremos que $\Psi$ é um homomorfismo. De fato, sejam $m$ e $n$ em $N$ tal que $m n$ está definido, isto é, existe um vértice entre eles. Portanto, ou ma e an estão definidos, ou $m b$ e $b n$ estão definidos. Faremos o caso $m a$ e $a n$ definidos pois o caso $m b$ e $b n$ definidos é análogo. Suponha então, ma e an definidos.

(1) Se $m$ e $n$ estão em $N_{a}$ então $\Psi(m)=\psi(m)$ e $\Psi(n)=\psi(n)$, e portanto $\Psi(m) \Psi(n)$ está definido e é igual a $\Psi(m n)$.

(2) Se $m$ está em $N_{a}$ e $n$ está em $N_{a b}$, então $\Psi(m)$ está em $M_{\psi(a)}$ e $n=n^{\prime} u$ para algum $n^{\prime}$ em $N_{a}$. Portanto, $\Psi(n)=\psi\left(n^{\prime}\right) x$ começa em $\psi(a)$. Portanto, $\Psi(m) \Psi(n)$ está definido e 


$$
\begin{aligned}
\Psi(m) \Psi(n) & =\psi(m) \psi\left(n^{\prime}\right) x \\
& =\psi\left(m n^{\prime}\right) x \\
& =\Psi\left(m n^{\prime} u\right) \\
& =\Psi(m n)
\end{aligned}
$$

(3) Se $m$ está em $N_{b a}$ e $n$ está em $N_{a b}$ então existem $n^{\prime}$ e $n^{\prime \prime}$ em $N_{a}$ tais que $m=u^{-1} n^{\prime}$ e $n=n^{\prime \prime} u$. Portanto, $\Psi(m)$ acaba em $\psi(a)$ e $\Psi(n)$ começa em $\psi(a)$. Logo, $\Psi(m) \Psi(n)$ está definido e

$$
\begin{aligned}
\Psi(m) \Psi(n) & =x^{-1} \psi\left(n^{\prime}\right) \psi\left(n^{\prime \prime}\right) x \\
& =x^{-1} \psi\left(n^{\prime} n^{\prime \prime}\right) x \\
& =\Psi\left(u^{-1} n^{\prime} n^{\prime \prime} u\right) \\
& =\Psi(m n)
\end{aligned}
$$

(4) Se $m$ está em $N_{b a}$ e $n$ está em $N_{a}$, então existe $n^{\prime}$ em $N_{a}$ tal que $m=u^{-1} n^{\prime}$. Como $\Psi(m)$ acaba em $\psi(a), \Psi(m) \Psi(n)$ está definido e

$$
\begin{aligned}
\Psi(m) \Psi(n) & =x^{-1} \psi\left(n^{\prime}\right) \psi(n) \\
& =x^{-1} \psi\left(n^{\prime} n\right) \\
& =\Psi\left(u^{-1} n^{\prime} n\right) \\
& =\Psi(m n)
\end{aligned}
$$

Daqui para a frente, vamos definir homomorfismos de grupóides como em (2.4.3) e (2.4.4), mas só escreveremos a forma (2.4.3).

A seguir, três exemplos que serão utilizados no capítulo 3.

Exemplo 2.4.7. Seja $G$ um grupóide conexo com $V(G)=\{a, b\}$. Considere os homomorfismos inclusão $i_{1}: D(a, b) \rightarrow G$ e $i_{2}: D(a, b) \rightarrow T(a, b)$. Então existe um grupóide $H$ e homomorfismos $j_{1}: G \rightarrow H$ e $j_{2}: T(a, b) \rightarrow H$ tais que $\left(H, j_{1}, j_{2}\right)$ é o push-out de 
$\left(i_{1}, i_{2}\right)$

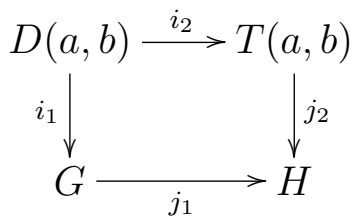

Demonstração. Seja $x$ em $T(a, b)$ a seta de $a$ a $b$ e seja $u$ em $G$ uma seta de $a$ a $b$ fixa.

Defina o grupóide $H$ como segue:

(1) $V(H)=\{a, b\}$

(2) $H_{a}=G_{a} *\langle t\rangle$, com $t$ de ordem infinita.

(3) $H_{b}, H_{a b}$ e $H_{b a}$ são definidos em função de $H_{a}$ e da seta $u$, como em (2.4.2).

A multiplicação parcial em $H$ é dada pela juxtaposição de setas. Observe que $G$ está incluido em $H G_{a} \subset G_{b}$ e $G_{b}, G_{a b}$ e $G_{b a}$ são definidos em função de $G_{a}$ e $u$, como em (2.4.2).

Defina $j_{1}: G \rightarrow H$ por $j_{1}(g)=g$. Defina $j_{2}: T(a, b) \rightarrow H$ por $j_{2}(x)=t u$. É claro que $j_{2}(a)=a$ e $j_{2}(b)=b$.

Vamos mostrar que $\left(H, j_{1}, j_{2}\right)$ é o push-out de $\left(i_{1}, i_{2}\right)$. Seja $K$ um grupóide e sejam $\varphi: G \rightarrow K$ e $\psi: T(a, b) \rightarrow K$ homomorfismos tais que

$$
D(a, b) \stackrel{i_{1}}{\longrightarrow} G \stackrel{\varphi}{\longrightarrow} K \text { e } D(a, b) \stackrel{i_{2}}{\longrightarrow} T(a, b) \stackrel{\psi}{\longrightarrow} K
$$

são homomorfismos iguais. Portanto, $\varphi(a)=\psi(a)$ e $\varphi(b)=\psi(b)$. Escreva $k=\psi(x)$ correspondente à seta em $K$ de $\varphi(a)$ a $\varphi(b)$.

Defina, estendendo de forma natural, o homomorfismo $\theta: H_{a} \rightarrow K_{\varphi(a)}$ por

$$
\theta(g)= \begin{cases}\varphi(g) & , \text { se } g \in G_{a} \\ k \varphi(u)^{-1} & , \text { se } g=t\end{cases}
$$

Defina $\theta(u)=\varphi(u)$. Pela proposição 2.4.6, $\theta$ é um homomorfismo. É evidente que 
$\varphi=\theta \circ j_{1}$, pois $G$ está incluido em $H$. Para provar que $\psi=\theta \circ j_{2}$, observe que

$$
\begin{aligned}
\theta\left(j_{2}(x)\right) & =\theta(t u) \\
& =k \varphi(u)^{-1} \varphi(u) \\
& =k
\end{aligned}
$$

Agora, provaremos que $\theta$ é único. Seja $\theta_{1}: H \rightarrow K$ outro homomorfismo tal que $\varphi=\theta_{1} \circ j_{1}$ e $\psi=\theta_{1} \circ j_{2}$. É claro que $\theta=\theta_{1}$ sobre $G_{a}$ como subconjunto de $H$, pois $\theta_{1} \circ j_{1}=\theta \circ j_{1}$. Além disso,

$$
\begin{aligned}
\theta_{1}(t) & =\theta_{1}(t u) \varphi(u)^{-1} \\
& =\theta_{1}\left(j_{2}(x)\right) \varphi(u)^{-1} \\
& =\psi(x) \varphi(u)^{-1} \\
& =\theta(t)
\end{aligned}
$$

pois $\theta_{1}(u)=\varphi(u)$ e $\theta_{1}$ é um homomorfismo. Segue então que $\theta=\theta_{1}$ sobre $H_{a}$, e pela proposição 2.4.6, $\theta=\theta_{1}$ sobre $H$.

O seguinte exemplo é uma generalização do exemplo anterior, e sua aplicação no capítulo 3 é muito importante.

Exemplo 2.4.8. Sejam $G$ e $H$ grupóides com $V(G)=\{a, b\}=V(H)$. Suponha $H$ conexo e considere $K$ um grupóide disconexo com $V(K)=\{a, b\}, K_{a}=H_{a}$ e $K_{b}=H_{b}$. Seja $i_{1}: K \rightarrow H$ o homomorfismo inclusão e $i_{2}: K \rightarrow G$ um homomorfismo qualquer. Então existe um grupóide $L$ e homomorfismos $j_{1}: H \rightarrow L$ e $j_{2}: G \rightarrow L$ tais que $\left(L, j_{1}, j_{2}\right)$ é o push-out de $\left(i_{1}, i_{2}\right)$

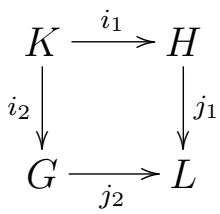

Demonstração. Observe que o homomorfismo $i_{2}$ pode ser visto como dois homomorfismos $\alpha: H_{a} \rightarrow G_{a}$ e $\beta: H_{b} \rightarrow G_{b}$ pois $K$ é disconexo. Como $H$ é conexo, escolha uma seta $u$ em $H$ de $a$ a $b$ e defina $\beta^{\prime}: H_{a} \rightarrow G_{b}$ por $\beta^{\prime}(h)=\beta\left(u^{-1} h u\right)$. 
Vamos construir $L$ conexo e com $V(L)=\{a, b\}$. Portanto, uma vez determinado $L_{a}$, escolhemos uma seta $x$ em $L$ de $a$ a $b$ e definimos $L_{a b}, L_{b a}$ e $L_{b}$ em função de $L_{a}$ e a seta $x$ (como na observação 2.4.4).

Quando $G$ for disconexo, definimos $L_{a}$ como sendo o push-out dos homomorfismos $\alpha$ e $\beta^{\prime}$.

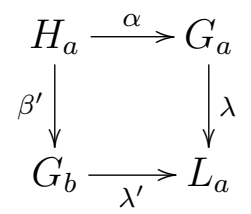

Note que a existência dos homomorfismos $\lambda$ e $\lambda^{\prime}$ está garantida pelo teorema 1.5.3.

Se $G$ for conexo, escolhemos una seta $v$ em $G$ de $a$ a $b$ e definimos $\beta^{\prime \prime}: H_{a} \rightarrow G_{a}$ por $\beta^{\prime \prime}(h)=v \beta\left(u^{-1} h u\right) v^{-1}$. Definimos $L_{a}$ sendo a (pseudo) extensão HNN de $G_{a}$ associada aos subgrupos $\alpha\left(H_{a}\right)$ e $\beta^{\prime \prime}\left(H_{a}\right)$, isto é,

$$
L_{a}=G_{a} *\langle t\rangle /\left\langle t^{-1} \beta^{\prime \prime}(h) t=\alpha(h): h \in H_{a}\right\rangle
$$

Seja $M$ um grupóide qualquer e $\theta: H \rightarrow M$ e $\epsilon: G \rightarrow M$ homomorfismos tais que

$$
K \stackrel{i_{1}}{\longrightarrow} H \stackrel{\theta}{\longrightarrow} M \text { e } K \stackrel{i_{2}}{\longrightarrow} G \stackrel{\epsilon}{\longrightarrow} M
$$

são homomorfismos iguais, isto é, $\theta \circ i_{1}=\epsilon \circ i_{2}$.

Suponha que $G$ é disconexo. O homomorfismo $\epsilon$, pode ser visto como dois homomorfismos $\varphi: G_{a} \rightarrow M_{\varphi(a)}$ e $\psi: G_{b} \rightarrow M_{\psi(b)}$. Note que $\theta=\varphi \circ \alpha$ sobre $H_{a}$ e $\theta=\psi \circ \beta$ sobre $H_{b}$. Escreva $w=\theta(u)$. Para $h$ em $H_{a}$ temos

$$
\begin{aligned}
\psi\left(\beta^{\prime}(h)\right) & =\psi\left(\beta\left(u^{-1} h u\right)\right) \\
& =\theta\left(u^{-1} h u\right) \\
& =w^{-1} \varphi(\alpha(h)) w
\end{aligned}
$$


Portanto, $\left(w^{-1}\right)_{\#} \circ \psi \circ \beta^{\prime}=\varphi \circ \alpha$ sobre $H_{a}$.

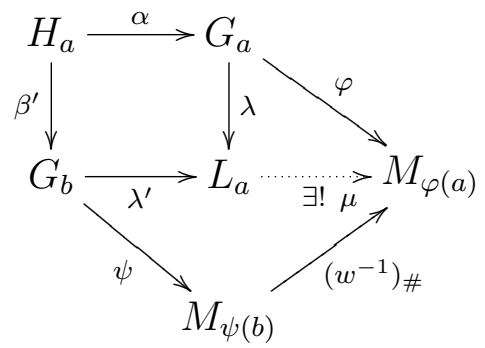

Como $\left(L_{a}, \lambda, \lambda^{\prime}\right)$ é o push-out de $\left(\alpha, \beta^{\prime}\right)$, então existe um único homomorfismo $\mu: L_{a} \rightarrow M_{\varphi(a)}$ tal que $\varphi=\mu \circ \lambda$ e $\left(w^{-1}\right)_{\#} \circ \psi=\mu \circ \lambda^{\prime}$.

Defina

$$
\xi: G \rightarrow L \text { por } \xi(g)= \begin{cases}\lambda(g) & , \text { se } g \in G_{a} \\ x_{\#}\left(\lambda^{\prime}(g)\right) & , \text { se } g \in G_{b}\end{cases}
$$

$\mathrm{e}$

$$
\tau: H \rightarrow L \text { por } \lambda(h)= \begin{cases}\lambda(\alpha(h)) & , \text { se } h \in H_{a} \\ x & , \text { se } h=u\end{cases}
$$

$\xi$ é um homomorfismo, pois $\lambda$ e $x_{\#} \circ \lambda^{\prime}$ são homomorfismos, e $\tau$ é um homomorfismo pela proposição 2.4.6. Observe que $\xi$ e $\tau$ não dependem de $\theta$ nem $\epsilon$.

Estenda $\mu$ para o homomorfismo $\mu: L \rightarrow M$ por $\mu(x)=w$ (ver proposição 2.4.6). Afirmamos que $(L, \tau, \xi)$ é o push-out de $\left(i_{1}, i_{2}\right)$. De fato, para $h$ em $H_{a}$ arbitrario, temos

$$
\begin{aligned}
\xi\left(i_{2}(h)\right) & =\xi(\alpha(h)) \\
& =\lambda(\alpha(h)) \\
& =\tau(h) \\
& =\tau\left(i_{1}(h)\right)
\end{aligned}
$$


$\mathrm{e}$

$$
\begin{aligned}
\xi\left(i_{2}\left(u^{-1} h u\right)\right) & =\xi\left(\beta\left(u^{-1} h u\right)\right) \\
& =\xi\left(\beta^{\prime}(h)\right) \\
& =x_{\#}\left(\lambda^{\prime}\left(\beta^{\prime}(h)\right)\right) \\
& =x^{-1} \lambda(\alpha(h)) x \\
& =\tau\left(u^{-1} h u\right) \\
& =\tau\left(i_{1}\left(u^{-1} h u\right)\right)
\end{aligned}
$$

Portanto, $\tau \circ i_{1}=\xi \circ i_{2}$. Para mostrar que $\theta=\mu \circ \tau$, basta considerar $h$ em $H_{a}$ e ver que

$$
\begin{aligned}
\mu(\tau(h)) & =\mu(\lambda(\alpha(h))) \\
& =\varphi(\alpha(h)) \\
& =\theta(h)
\end{aligned}
$$

e que $\mu(\tau(u))=\mu(x)=\theta(u)$, e aplicar a proposição 2.4.6. Agora, mostraremos que $\epsilon=\mu \circ \xi$. Seja $g$ em $G_{a}$, então

$$
\begin{aligned}
\mu(\xi(g)) & =\mu(\lambda(g)) \\
& =\varphi(g) \\
& =\epsilon(g)
\end{aligned}
$$

Seja $g$ em $G_{b}$, então

$$
\begin{aligned}
\mu(\xi(g)) & =\mu\left(x^{-1} \lambda^{\prime}(g) x\right) \\
& =w^{-1} \mu\left(\lambda^{\prime}(g)\right) w \\
& =w^{-1}\left(w^{-1}\right)_{\#}(\psi(g)) w \\
& =\psi(g) \\
& =\epsilon(g)
\end{aligned}
$$

Finalmente, mostraremos que $\mu$ é único. Seja $\mu_{1}: L \rightarrow M$ um homomorfismo tal que 
$\mu_{1} \circ \tau=\theta$ e $\mu_{1} \circ \xi=\epsilon$. Observe que para $g$ em $G_{a}$ temos

$$
\begin{aligned}
\mu_{1}(\lambda(g)) & =\mu_{1}(\xi(g)) \\
& =\epsilon(g) \\
& =\varphi(g)
\end{aligned}
$$

e que para $g$ em $G_{b}$ temos

$$
\begin{aligned}
\mu_{1}\left(\lambda^{\prime}(g)\right) & =\mu_{1}\left(x x_{\#}\left(\lambda^{\prime}(g)\right) x^{-1}\right) \\
& =\mu_{1}(x) \mu_{1}(\xi(g)) \mu_{1}(x)^{-1} \\
& =\mu_{1}(\tau(u)) \epsilon(g) \mu_{1}(\tau(g))^{-1} \\
& =w \psi(g) w^{-1} \\
& =\left(w^{-1}\right)_{\#}(\psi(g))
\end{aligned}
$$

Portanto, $\mu_{1}=\mu$ sobre $L_{a}$. Além disso, $\mu_{1}(x)=\mu(x)=w$, pela proposição $2.4 .6, \mu_{1}=\mu$ em $L$.

Agora, suponha $G$ conexo. Escolha uma seta $v$ em $G$ de $a$ a $b$, e defina $\beta^{\prime \prime}: H_{a} \rightarrow$ $G_{a}$ por $\beta^{\prime \prime}(h)=v \beta^{\prime}(h) v^{-1}$. Agora o homomorfismo $\epsilon: G \rightarrow M$ é definido por um homomorfismo $\varphi: G_{a} \rightarrow M_{\varphi(a)}$ e um elemento $y=\epsilon(v)$ em $M_{\varphi(a) \theta(b)}$. Assim, $\epsilon$ fica definido em $G$ como em (2.4.4) (ver proposição 2.4.6). Usando que $\epsilon \circ i_{1}=\theta \circ i_{2}$, temos para $h$ em $H_{a}$

$$
\begin{aligned}
\theta\left(u^{-1} h u\right) & =\epsilon\left(\beta\left(u^{-1} h u\right)\right) \\
& =y^{-1} \epsilon\left(\beta^{\prime \prime}(h)\right) y \\
& =y^{-1} \varphi\left(\beta^{\prime \prime}(h)\right) y
\end{aligned}
$$

Portanto, $\varphi(\alpha(h))=\left(y w^{-1}\right)^{-1} \varphi\left(\beta^{\prime \prime}(h)\right)\left(y w^{-1}\right)$, para todo $h$ em $H_{a}$. Pela propriedade universal das extensões HNN (proposição 1.5.9), existe um único homomorfismo $\mu: L_{a} \rightarrow M_{\varphi(a)}$ tal que $\mu \circ j=\varphi$ e $\mu(t)=y w^{-1}$, onde $j: G_{a} \rightarrow L_{a}$ é o homomorfismo 
induzido pela inclusão $G_{a} \hookrightarrow G_{a} *\langle t\rangle$.

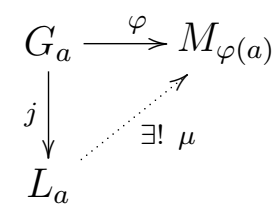

Estenda $\mu$ a um homomorfismo $\mu: L \rightarrow M$ por $\mu(x)=w$ (isto esta garantido pela proposição 2.4.6). Considere os homomorfismos

$$
\xi: G \rightarrow L \text { definido por } \xi(g)= \begin{cases}j(g) & , \text { se } g \in G_{a} \\ t x & , \text { se } g=v\end{cases}
$$

$\mathrm{e}$

$$
\tau: H \rightarrow L \text { definido por } \tau(h)= \begin{cases}j(\alpha(h)) & , \text { se } h \in H_{a} \\ x & , \text { se } h=u\end{cases}
$$

$\xi$ e $\tau$ são homomorfismos pela proposição 2.4.6, e não dependem de $\theta$ nem $\epsilon$. Afirmamos que $(L, \tau, \xi)$ é o push-out de $\left(i_{1}, i_{2}\right)$. De fato, se $h$ está em $H_{a}$

$$
\begin{aligned}
\tau\left(i_{1}(h)\right) & =\tau(h) \\
& =j(\alpha(h)) \\
& =\xi(\alpha(h)) \\
& =\xi\left(i_{2}(h)\right)
\end{aligned}
$$


e se $h$ está em $H_{b}$, existe $h^{\prime}$ em $H_{a}$ tal que $h=u^{-1} h^{\prime} u$ em $H$, assim

$$
\begin{aligned}
\tau\left(i_{1}(h)\right) & =\tau(h) \\
& =\tau\left(u^{-1} h^{\prime} u\right) \\
& =x^{-1} j\left(\alpha\left(h^{\prime}\right)\right) x \\
& =x^{-1} t^{-1} j\left(\beta^{\prime \prime}\left(h^{\prime}\right)\right) t x \\
& =\xi\left(v^{-1} \beta^{\prime \prime}\left(h^{\prime}\right) v\right) \\
& =\xi\left(\beta\left(u^{-1} h^{\prime} u\right)\right) \\
& =\xi(\beta(h)) \\
& =\xi\left(i_{2}(h)\right)
\end{aligned}
$$

Portanto, $\tau \circ i_{1}=\xi \circ i_{2}$.

Para mostrar que $\theta=\mu \circ \tau$, considere $h$ em $H_{a}$ e observe que

$$
\mu(\tau(h))=\mu(j(\alpha(h)))=\varphi(\alpha(h))=\theta(h)
$$

e $\mu(\tau(u))=\mu(x)=\theta(u)$. De maneira análoga, para mostrar que $\epsilon=\mu \circ \xi$, observe que dado $g$ em $G_{a}$, temos

$$
\mu(\xi(g))=\mu(j(g))=\varphi(g)=\epsilon(g)
$$

e $\mu(\xi(v))=\mu(t x)=y=\epsilon(v)$.

Agora, demonstraremos que o homomorfismo $\mu$ é único. Suponha exista um homomorfismo $\mu_{1}: L \rightarrow M$ tal que $\mu_{1} \circ \tau=\theta$ e $\mu_{1} \circ \xi=\epsilon$. Observe que para $g$ em $G_{a}$, como subconjunto de $L$, temos

$$
\mu(g)=\mu(j(g))=\varphi(g)=\epsilon(g)=\mu_{1}(\xi(g))=\mu_{1}(j(g))=\mu_{1}(j(g))=\mu_{1}(g)
$$

e

$$
\mu(t)=y w^{-1}=\mu_{1}(\xi(v)) \mu_{1}(\tau(u))^{-1}=\mu_{1}(t x) \mu_{1}(x)^{-1}=\mu_{1}(t)
$$

Portanto, $\mu=\mu_{1}$ sobre $L_{a}$. É fácil ver que $\mu(x)=\mu_{1}(x)$, assim, $\mu=\mu_{1}$ sobre $L$ (proposição 2.4.6). 
O seguinte exemplo é usado para obter um corolário do Teorema de Seifert-Van Kampen que será de muita importância devido a suas aplicações.

Exemplo 2.4.9. Suponha que temos um push-out ponteado de grupóides

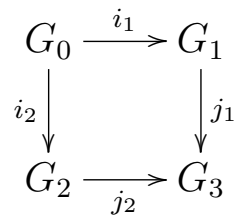

isto é, $V\left(G_{0}\right)=V\left(G_{1}\right) \cap V\left(G_{2}\right), V\left(G_{3}\right)=V\left(G_{1}\right) \cup V\left(G_{2}\right) e, i_{1}, i_{2}, j_{1}$ e $j_{2}$ são os homorfismos inclusão. Seja $S_{3} \subset V\left(G_{3}\right)$. Para $r=0,1,2$, seja $S_{r}=S_{3} \cap V\left(G_{r}\right)$ e suponha que $S_{r}$ intercepta a cada componente de $G_{r}$. Para $r=0,1,2,3$, seja $H_{r}=\left(G_{r}\right)_{S_{r}}$, então temos o quadrado push-out

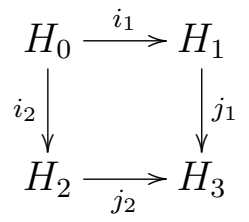

Supondo, além das hipóteses anteriores, que $G_{r}$ é conexo para $r=0,1,2$, (portanto, $G_{3}$ é conexo também), e dado a em $V\left(G_{0}\right)$, temos um quadrado push-out de grupos

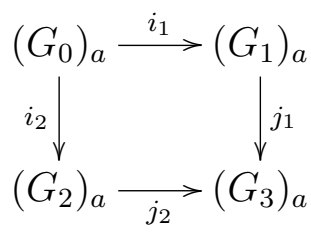

Demonstração. Seja $H$ um grupóide e para $r=1,2$, considere homomorfismos $\varphi_{r}: H_{r} \rightarrow H$ tais que $\varphi_{1} \circ i_{1}=\varphi_{2} \circ i_{2}$.

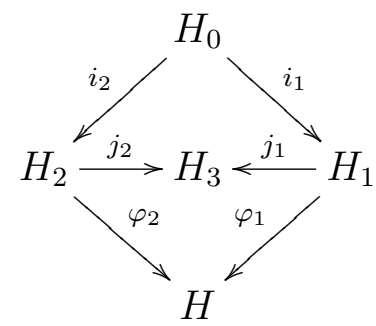

Vamos estender os homomorfismos $\varphi_{1}$ e $\varphi_{2}$ de maneira adequada para homomorfismos 
$\psi_{1}: G_{1} \rightarrow H$ e $\psi_{2}: G_{2} \rightarrow H$ tais que $\psi_{1} \circ i_{1}=\psi_{2} \circ i_{2}$. Para cada vértice $v$ em $G_{r}$ escolha uma seta $x_{r v}$ saindo de $S_{r}$ e chegando em $v$, e se $v$ está em $S_{r}$ escolha $x_{r v}=v$, para $r=1,2$. A existência de $x_{r v}$ está garantida, pois $S_{r}$ intercepta a cada componente de $G_{r}$.

Dado $v$ em $V\left(G_{0}\right)$, considere a seta $x_{0 v}$ em $G_{0}$ de algum ponto de $S_{0}$ a $v$. Para $r=1,2$, escreva $x_{r v}=i_{r}\left(x_{0 v}\right)$ em $G_{r}$, e defina para uma seta $g$ em $G_{r}$ de $a$ a $b$ a função

$$
\psi_{r}(g)= \begin{cases}\varphi_{r}\left(x_{r a} g x_{r b}^{-1}\right) & , \text { se } a, b \notin S_{r} \\ \varphi_{r}\left(x_{r a} g\right) & , \text { se } a \notin S_{r}, b \in S_{r} \\ \varphi_{r}\left(g x_{r b}^{-1}\right) & , \text { se } a \in S_{r}, b \notin S_{r} \\ \varphi_{r}(g) & , \text { se } a, b \in S_{r}\end{cases}
$$

Pela proposição 2.3.4, $\psi_{r}$ é um homomorfismo, para $r=1,2$. Além disso, pela definição de $\psi_{r}$ e a escolha das setas $x_{r v}$, é fácil ver que $\psi_{1} \circ i_{1}=\psi_{2} \circ i_{2}$.

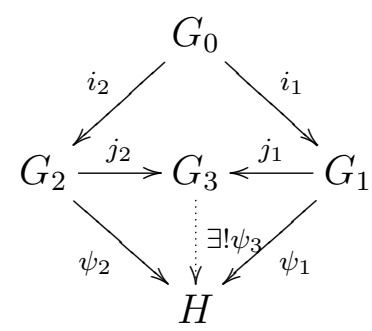

Como $\left(G, j_{1}, j_{2}\right)$ é o push-out de $\left(i_{1}, i_{2}\right)$, existe um único homomorfismo $\psi_{3}: G_{3} \rightarrow H$ tal que $\psi_{3} \circ j_{1}=\psi_{1}$ e $\psi_{3} \circ j_{2}=\psi_{2}$. Considere o homomorfismo $\varphi_{3}=\left.\psi_{3}\right|_{H_{3}}$, então, é claro que $\varphi_{3} \circ j_{1}=\varphi_{1}$ e $\varphi_{3} \circ j_{2}=\varphi_{2}$.

Queremos provar que $\varphi_{3}$ é único. Seja $\varphi: H_{3} \rightarrow H$ um homomorfismo tal que $\varphi \circ j_{1}=\varphi_{1}$ e $\varphi \circ j_{2}=\varphi_{2}$. Estenda $\varphi$ para o homomorfismo $\psi: G_{3} \rightarrow H$, definindo para uma seta $g$ em $G_{3}$ de $a$ a $b$

$$
\psi(g)= \begin{cases}\varphi\left(x_{3 a} g x_{3 b}^{-1}\right) & , \text { se } a, b \notin S_{3} \\ \varphi\left(x_{3 a} g\right) & , \text { se } a \notin S_{3}, b \in S_{3} \\ \varphi\left(g x_{3 b}^{-1}\right) & , \text { se } a \in S_{3}, b \notin S_{3} \\ \varphi(g) & , \text { se } a, b \in S_{3}\end{cases}
$$

onde $x_{3 v}=j_{r}\left(x_{r v}\right)$ para cada vértice $v$ em $V\left(G_{3}\right)$ e $r=1$, 2. É fácil ver que $\psi \circ j_{r}=\psi_{r}$ 
para $r=1,2$, portanto, $\psi=\psi_{3}$. Segue que $\varphi_{3}=\varphi$. 


\section{Capítulo}

\section{3}

\section{Elementos de Topologia e Topologia}

\section{Algébrica}

\subsection{Topologia Fraca e Topologia Quociente}

\section{Lema 3.1.1 (Da Colagem).}

(i) Sejam $X$ e $Y$ conjuntos. Seja $\left\{X_{\alpha}\right\}_{\alpha \in \Lambda}$ uma familia de subconjuntos de $X$ tais que $X=\bigcup_{\alpha \in \Lambda} X_{\alpha}$. Seja $\left\{f_{\alpha}: X_{\alpha} \rightarrow Y / \alpha \in \Lambda\right\}$ uma família de funções tais que $\left.f_{\alpha}\right|_{X_{\alpha} \cap X_{\beta}}=\left.f_{\beta}\right|_{X_{\alpha} \cap X_{\beta}}$, para todo $\alpha$ e $\beta$ em $\Lambda$. Então, existe uma única função $f: X \rightarrow Y$ tal que $\left.f\right|_{X_{\alpha}}=f_{\alpha}$, para todo $\alpha$ em $\Lambda$.

(ii) Nas mesmas hipóteses de (i). Suponha que $X$ e $Y$ são espaços topológicos, e que $f_{\alpha}$ é contínua para todo $\alpha$ em $\Lambda$. Além disso, suponha que, ou existe um número finito de $X_{\alpha}$ 's e que são fechados em $X$, ou que cada $X_{\alpha}$ é aberto. Então $f: X \rightarrow Y$ como em (i), é contínua.

Definição 3.1.2. Seja $\left\{\left(X_{\alpha}, \tau_{\alpha}\right)\right\}_{\alpha \in \Lambda}$ uma coleção de espaços topológicos de um conjunto $X$. Defina

$$
\tau=\left\{A \subset X: A \cap X_{\alpha} \in \tau_{\alpha}, \forall \alpha \in \Lambda\right\}
$$

$\tau$ é uma topologia sobre $X$ chamada topologia fraca em $X$ induzida por $\left\{\tau_{\alpha}\right\}_{\alpha \in \Lambda}$. 
Na definição 3.1.2, se $X_{\alpha} \cap X_{\beta}=\emptyset$, para todo $\alpha, \beta$ em $\Lambda$ com $\alpha \neq \beta$, então o espaço $X$ (com a topologia fraca) é conhecido como a união disjunta da família de espaços $\left\{X_{\alpha}\right\}_{\alpha \in \Lambda}$ e sería denotado por

$$
X=\bigcup_{\alpha \in \Lambda} X_{\alpha}
$$

Se, em vez de ter que a família $\left\{X_{\alpha}\right\}_{\alpha \in \Lambda}$ é disjunta, nós temos que $X_{\alpha} \cap X_{\beta}=\{p\}$ para algum ponto $p$ em $X$ e para todo $\alpha$ e $\beta$ em $\Lambda \operatorname{com} \alpha \neq \beta$, então $X$ é dito ser junção da família de espaços $\left\{X_{\alpha}\right\}_{\alpha \in \Lambda}$ em $p$ e é denotada por

$$
X=\bigvee_{\alpha \in \Lambda} X_{\alpha}
$$

Definição 3.1.3. Seja $\left\{X_{\alpha}\right\}_{\alpha \in \Lambda}$ uma família de conjuntos qualquer. Considere o conjunto

$$
X=\bigcup_{\alpha \in \Lambda} X_{\alpha}=\bigcup_{\alpha \in \Lambda}\{\alpha\} \times X_{\alpha}
$$

$X$ é chamado união disjunta livre da familia de conjuntos $\left\{X_{\alpha}\right\}_{\alpha \in \Lambda}$.

Observação 3.1.4. Podemos fornecer a uma união disjunta livre de conjuntos a topologia fraca, sempre que tais conjuntos sejam espaços topológicos. Neste caso, vamos nos referir ao espaço como espaço da união disjunta livre.

Observação 3.1.5. Na definição 3.1.2, um conjunto $C$ é fechado em $X$ com a topologia fraca $\tau$ se, e somente se, $C \cap X_{\alpha}$ é fechado em $\tau_{\alpha}$, para todo $\alpha$ em $\Lambda$. Em particular, se cada $\left(X_{\alpha}, \tau_{\alpha}\right)$ é uma espaço $T_{1}$, então $(X, \tau)$ também é $T_{1}$.

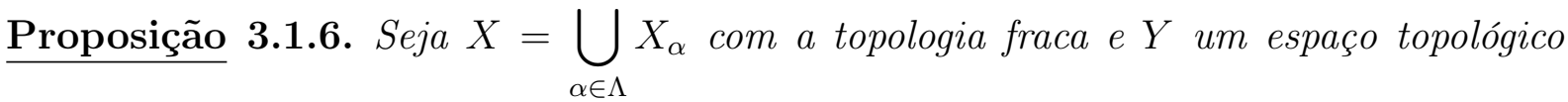
qualquer. Então, uma função $f: X \rightarrow Y$ é contínua, se e somente se, $\left.f\right|_{X_{\alpha}}$ é contínua com a topologia de $X_{\alpha}$, para todo $\alpha$ em $\Lambda$.

Definição 3.1.7. Seja $(X, \tau)$ um espaço topológico e seja $p: X \rightarrow Y$ uma aplicação sobrejetora. Defina

$$
\tau_{Y}=\left\{B \subset Y: p^{-1}(B) \in \tau\right\}
$$

$\tau_{Y}$ é uma topologia em $Y$ chamada topologia quociente (ou da identificação) sobre $Y$ induzida por $p$. 
Definição 3.1.8. Sejam X e $Y$ espaços topológicos. Uma função contínua e sobrejetora $p: X \rightarrow Y$ é chamada aplicação de identificação quando a topologia em $Y$ é a topologia quociente induzida por p.

Proposição 3.1.9. Se $p: X \rightarrow Y$ é uma aplicação sobrejetora, contínua e aberta (ou fechada), então p é uma identificação.

Seja $X$ um espaço topológico e $\equiv$ uma relação de equivalência em $X$. Seja $Y$ o conjunto das classes de equivalência dos elementos de $X$ sob a relação $\equiv$. Considere a aplicação natural $p: X \rightarrow Y$ definida por $p(x)=\bar{x}$, onde $\bar{x}$ é a classe de equivalência de $x . p$ é sobrejetora, portanto, podemos dar a $Y$ a topologia quociente. Vamos denotar este espaço topológico por $X / \equiv$, e vamos chamá-lo de espaço obtido de $X$ por identificação dos pontos de cada classe de equivalência sob $\equiv$.

Proposição 3.1.10. Seja $\equiv$ uma relação de equivalência sobre um conjunto $X$. Seja $Z$ um conjunto qualquer e $f: X \rightarrow Z$ uma função contínua. Seja $Y$ o conjunto quociente $X / \equiv$ e seja $p: X \rightarrow Y$ a projeção natural.

(i) Então, existe uma função $g: Y \rightarrow Z$ tal que $f=g \circ p$ se, e somente se, $f\left(x_{1}\right)=$ $f\left(x_{2}\right)$, sempre que $x_{1} \equiv x_{2}$.

(ii) Sejam $X$ e $Z$ espaços topológicos e seja $Y$ com a topologia da identificação. Seja $g$ como em (i), então g é contínua se, e somente se, $f$ é contínua.

Proposição 3.1.11. Seja $X$ um espaço compacto e $Y$ um espaço de Hausdorff. Se $f: X \rightarrow Y$ é contínua e bijetora então $f$ é um homeomorfismo.

Proposição 3.1.12. Se $X$ é compacto então $X / \equiv$ é compacto.

Proposição 3.1.13. Seja $X$ um espaço compacto e $Y$ um espaço Hausdorff. Seja $f$ : $X \rightarrow Y$ uma aplicação contínua e sobrejetora. Então $Y$ tem a topologia da identificação.

\subsection{Adjuntando Espaços mediante Funções}

Sejam $X$ e $Y$ espaços topológicos e $A$ um sub-espaço de $X$. Seja $f: A \rightarrow Y$ uma função contínua. Construa o espaço, denotado por $Y \cup_{f} X$, a partir do espaço $X \bigcup \dot{U} Y$ 
mediante uma identificação dos pontos $a \operatorname{com} f(a)$, para todo $a$ em $A$. O espaço $Y \cup_{f} X$ é chamado espaço obtido adjuntando $X$ a $Y$ mediante $f$. Observe que as classes de equivalência de $X \bigcup \dot{U} Y$ sob esta identificacão, são os conjuntos da forma

- $\{y\} \cup f^{-1}\{y\}$, para $y \operatorname{em~} f(A)$.

- $\{x\}$ quando $x$ está em $(X \backslash A) \bigcup \dot{\bigcup}(Y \backslash f(A))$

Vamosnos referir a estes conjuntos mediante algum representante, assim, escreveremos $\{y\} \cup f^{-1}\{y\}$ simplesmente por $y$ ou, $x$, quando $y$ for a imagem de $x$ sob $f$, e $x$ por $\{x\}$ quando $x$ esteja em $(X \backslash A) \dot{U}(Y \backslash f(A))$.

Teorema 3.2.1. Sejam X e $Y$ espaços topológicos e A um sub-espaço de X. Seja $f: A \rightarrow Y$ uma função contínua e considere $\pi: X \dot{\bigcup} Y \rightarrow Y \cup_{f} X$ a projeção natural. Então, $Y$ é mergulhado em $Y \cup_{f} X$ como um conjunto fechado, e $X$ é mergulhado em $Y \cup_{f} X$ como um conjunto aberto.

Proposição 3.2.2. Seja $f: X \rightarrow Y$ a aplicação identificação de espaços topológicos. Seja $Z$ um espaço localmente compacto. Então, $F: X \times Z \rightarrow Y \times Z$ dada por $F(x, z)=(f(x), z)$ é uma identificação.

A proposição 3.2.2 é importante para construir homotopias entre caminhos (ver definições 3.3.1 e 3.3.2) do espaço $Y \cup_{f} X$.

\subsection{Caminhos e Homotopias}

Definição 3.3.1. Um caminho num espaço $X$ é uma aplicação contínua $f:[0,1] \rightarrow X$.

(i) Se $f(t)=x_{0}$, para todo $t \in[0,1]$, então chamamos a $f$ caminho constante em $x_{0}$. Neste caso denotaremos $f$ por $e_{x_{0}}$.

(ii) Se $f(0)=a$ e $f(1)=b$, dizemos que $f$ é um caminho de a até b, ou um caminho com ponto inicial a e com ponto final $b$, ou começando em a e terminando em $b$.

(iii) Se $f(0)=f(1)=a, f$ é chamado laço em $X$ baseado em a. 
Definição 3.3.2. Sejam $f, g:[0,1] \rightarrow X$ dois caminhos em $X$ de a até b. Suponha que exista uma aplicação contínua $F:[0,1] \times[0,1] \rightarrow X$ tal que

$$
\begin{array}{ll}
F(t, 0)=f(t) & , 0 \leq t \leq 1 \\
F(t, 1)=g(t) & , 0 \leq t \leq 1
\end{array}
$$

$e$

$$
\left.\begin{array}{l}
F(0, u)=a \\
F(1, u)=b
\end{array}\right\} 0 \leq u \leq 1
$$

então, dizemos que $f$ é homotópico por caminhos a $g$. A aplicação $F$ é chamada homotopia por caminhos entre $f$ e $g$. Quando $f$ for homotópico por caminhos a $g$, escreveremos $f \simeq g$.

Proposição 3.3.3. Ser homotópico por caminhos é uma relação de equivalência.

Vamos denotar a classe de homotopia de um caminho $f$ em $X$ por $[f]$, isto é,

$$
[f]=\{g: g \text { é um caminho em } X \text { com } g \simeq f\}
$$

Definição 3.3.4. Sejam $f, g:[0,1] \rightarrow X$ caminhos num espaço $X$ tais que $f(1)=g(0)$. Definimos o caminho $f * g:[0,1] \rightarrow X$ por

$$
(f * g)(t)= \begin{cases}f(2 t) & , 0 \leq t \leq \frac{1}{2} \\ g(2 t-1) & , \frac{1}{2} \leq t \leq 1\end{cases}
$$

$f * g$ é chamado o caminho produto (ou simplesmente, produto) entre $f$ e $g$. Dizemos que $f * g$ está definido sempre que $g(0)=f(1)$.

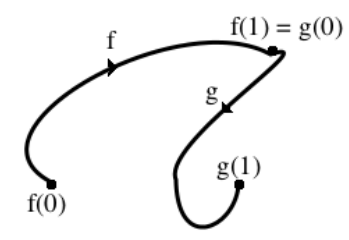

Figura 3.1: O caminho produto dos caminhos $f$ e $g$. 
Proposição 3.3.5. O produto $*$ satisfaz

(1) $(f * g) * h \simeq f *(g * h)$, para caminhos $f, g$ e $h$ tais que $f * g$ e $g * h$ estejam definidos.

(2) $e_{a} * f \simeq f \simeq f * e_{b}$, quando f for um caminho de a até $b$.

Definição 3.3.6. Seja $f:[0,1] \rightarrow X$ um caminho de a até b. Definimos $\bar{f}:[0,1] \rightarrow X$ por $\bar{f}(t)=f(1-t) . \bar{f}$ é chamado caminho inverso de $f$.

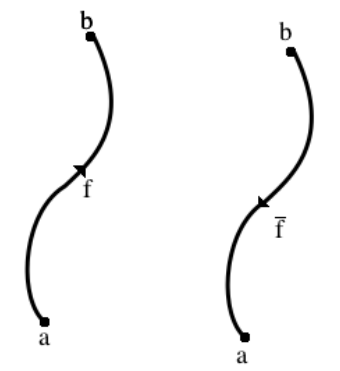

Figura 3.2: O caminho inverso de $f$.

Observação 3.3.7. Sejam $f$ e $g$ caminhos tais que $f * g$ está definido, então $\bar{g} * \bar{f}$ está definido e $\overline{f * g}=\bar{g} * \bar{f}$. Além disso, $\overline{\bar{f}}=f$.

Lema 3.3.8. Seja $f$ um caminho de a até b. Então, $f * \bar{f} \simeq e_{a} e \bar{f} * f \simeq e_{b}$

Lema 3.3.9. Sejam $f, f^{\prime}, g$ e $g^{\prime}$ caminhos tais que $f * f^{\prime}$ e $g * g^{\prime}$ estão definidos. Se $f \simeq g$ e $f^{\prime} \simeq g^{\prime}$ então $f * f^{\prime} \simeq g * g^{\prime}$.

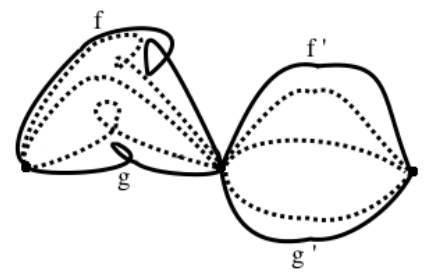

Figura 3.3: $f * f^{\prime} \simeq g * g^{\prime}$ 


\subsection{O Grupóide Fundamental e o Grupo Fundamental}

Já vimos que o conjunto dos caminhos num espaço topológico $X$ pode ser dotado de uma multiplicação parcial, a saber, o produto * de caminhos (ver definição 3.3.4). Sob esta multiplicação não há inversos. O máximo que podemos conseguir em $X$, é que para cada caminho $f$ existe um caminho $\bar{f}$ tal que $f * \bar{f}$ e $\bar{f} * f$ são homotópicos a caminhos constantes (lema 3.3.8). Isto sugere que $*$ induz uma multiplicação parcial sobre as classes de equivalência de caminhos em $X$ que é associativa, tem identidades e inversos.

Denotemos por $\Upsilon(X)$ o conjunto de todas as classes de homotopia por caminhos de caminhos em $X$, isto é,

$$
\Upsilon(X)=\{[f]: f:[0,1] \rightarrow X \text { é um caminho em } \mathrm{X}\}
$$

Dotemos $\Upsilon(X)$ de uma multiplicação parcial $*$ definida por $[f] *[g]=[f * g]$. Este produto está definido para aqueles caminhos tais que $f * g$ esteja definido. Então

(1) Se $([f] *[g]) *[h]$ ou $[f] *([g] *[h])$ está definido, então o outro também está definido e são iguais. Além disso, se $[f] *[g]$ e $[g] *[h]$ estão definidos então $([f] *[g]) *[h]($ e, $[f] *([g] *[h]))$ está definido.

(2) Sendo $f$ um caminho de $a$ até $b$ temos

(2.1) $\left[e_{a}\right] *[f]=[f]$ e $[f] *\left[e_{b}\right]=[f]$ (ver proposição 3.3.5).

$(2.2)[f] *[\bar{f}]=\left[e_{a}\right]$ e $[\bar{f}] *[f]=\left[e_{b}\right]$, ou seja, $[f]^{-1}=[\bar{f}]$ (ver lema 3.3.8).

Portanto, $(\Upsilon(X), *)$ tem estrutura de grupóide, e é chamado grupóide fundamental do espaço $X$.

O conjunto dos vértices de $\Upsilon(X)$ é um subconjunto de $\Upsilon(X)$ em bijeção com $X$, é por isso que vamos considerar como conjunto de vértices de $\Upsilon(X)$ o próprio $X$. O grupo vértice de $\Upsilon(X)$ em um vértice $a$ em $X$, é denotado por $\pi_{1}(X, a)$ e é chamado grupo fundamental de $X$ baseado em $a$, e corresponde ao conjunto das classes de homotopia dos laços em $X$ baseados em $a$, isto é,

$$
\pi_{1}(X, a)=\{[f]: f:[0,1] \rightarrow X \text { é um laço em } X \text { baseado em } a\}
$$

As componentes do grupóide $\Upsilon(X)$ são as componentes conexas por caminhos de $X$. Em particular, $X$ é conexo por caminhos, se e somente se, $\Upsilon(X)$ é um grupóide conexo. 
Neste caso, $\pi_{1}(X, a)$ é isomorfo a $\pi_{1}(X, b)$ para todo $a$ e $b$ em $X$. De fato, seja $\lambda$ um caminho em $X$ de $a$ até $b$. Definamos $\lambda_{\#}: \pi_{1}(X, a) \rightarrow \pi_{1}(X, b)$ por $\lambda_{\#}[f]=[\bar{\lambda}] *[f] *[\lambda]$. É fácil ver que $\lambda_{\#}$ é um isomorfismo.

Quando $X$ for conexo por caminhos, vamos denotar o grupo $\pi_{1}(X, a)$ simplesmente por $\pi_{1}(X)$, para todo $a$ em $X$, pois este grupo não depende do ponto base.

Sejam $X$ e $Y$ espaços topológicos e $\varphi: X \rightarrow Y$ uma função contínua. Se $f$ e $g$ são caminhos homotópicos em $X$, então $\varphi \circ f$ e $\varphi \circ g$ são caminhos homotópicos em $Y$ mediante a homotopia $\varphi \circ F$, onde $F$ é uma homotopia entre $f$ e $g$. Portanto, a aplicação $\varphi_{*}: \Upsilon(X) \rightarrow \Upsilon(Y)$ definida por $\varphi_{*}([f])=[\varphi \circ f]$ está bem definida. É fácil ver que $\varphi_{*}$ é um homomorfismo. $\varphi_{*}$ é chamado homomorfismo induzido por $\varphi$. Note que se $\varphi(a)=b$, então $\varphi_{*}$ induz um homomorfismo de $\pi_{1}(X, a)$ em $\pi_{1}(Y, b)$, que também denotaremos por $\varphi_{*}$.

\section{Proposição 3.4.1.}

(1) Sejam $\varphi: X \rightarrow Y$ e $\psi: Y \rightarrow Z$ funções contínuas. Então $(\psi \circ \varphi)_{*}=\psi_{*} \circ \varphi_{*}$.

(2) Seja $I d_{X}: X \rightarrow X$ a função identidade de $X$. Então $\left(I d_{X}\right)_{*}=I d_{\Upsilon(X)}$.

Corolário 3.4.2. Se $X$ e $Y$ são espaços homeomorfos então $\Upsilon(X)$ e $\Upsilon(Y)$ são grupóides isomorfos $e, \pi_{1}(X, a)$ e $\pi_{1}(Y, b)$ são grupos isomorfos, onde $b$ é a imagen de a sob o homeomorfismo dado.

Proposição 3.4.3. Sejam $X$ e $Y$ espaços topológicos. Então, $\Upsilon(X \times Y)$ é isomorfo a $\Upsilon(X) \times \Upsilon(Y)$ e $\pi_{1}(X \times Y,(x, y))$ é isomorfo a $\pi_{1}(X, x) \times \pi_{1}(Y, y)$.

Definição 3.4.4. Sejam $\varphi$ e $\psi$ aplicações contínuas de $X$ em $Y$. Dizemos que $\varphi$ é homotópica a $\psi$ se existe uma aplicação contínua $\phi: X \times[0,1] \rightarrow Y$ tal que

$$
\left.\begin{array}{l}
\phi(x, 0)=\varphi(x) \\
\phi(x, 1)=\psi(x)
\end{array}\right\} \text { para todo } x \text { em } X
$$

Dizemos que $\phi$ é uma homotopia de $\varphi$ a $\psi$ e escrevemos $\varphi \simeq \psi$.

Proposição 3.4.5. Homotopia de aplicações contínuas de um espaço $X$ num espaço $Y$ é uma relação de equivalência. 
Definição 3.4.6. Sejam $X$ e $Y$ espaços topológicos. Seja $C$ subconjunto de $X$ e $\varphi, \psi: X \rightarrow Y$ funções contínuas. Se existir uma homotopia $\phi: X \times[0,1] \rightarrow Y$ de $\varphi$ a $\psi$ tal que para todo $c \in C$ e $t \in[0,1]$ temos

$$
\phi(c, t)=\varphi(c)=\psi(c)
$$

então dizemos que $\varphi$ e $\psi$ são homotópicas relavitamente a $C$, e escrevemos $\varphi \simeq$

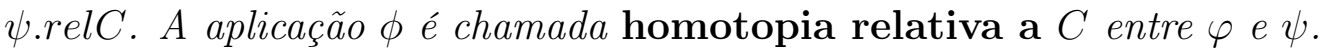

Definição 3.4.7. Uma aplicação contínua $\varphi: X \rightarrow Y$ é chamada equivalência de homotopia entre os espaços $X$ e $Y$, se existir uma aplicação contínua $\psi: Y \rightarrow X$ tal que $\psi \circ \varphi \simeq I d_{X}$ e $\varphi \circ \psi \simeq I d_{Y}$. Dizemos que o espaço $X$ é homotopicamente equivalente $a Y$, e que $\psi$ é inversa homotópica $d e \varphi$.

Observação 3.4.8. Ser homotopicamente equivalente é uma relação de equivalência sobre os espaços topológicos.

Observação 3.4.9. Suponha que $X$ é homotopicamente equivalente a $Y$. Então:

(i) $\Upsilon(X)$ é isomorfo a $\Upsilon(Y)$.

(ii) Se $X$ for conexo por caminhos, então $Y$ é conexo por caminhos.

Definição 3.4.10. Um espaço $X$ é chamado simplesmente conexo se é conexo por caminhos e $\pi_{1}(X)$ é trivial.

Seja $X$ simplesmente conexo, então todo laço é homotópico ao laço constante, portanto, dois caminhos qualquer em $X$ de $a$ até $b$ são homotópicos. Assim, existe uma única classe de homotopia de caminhos de $a$ até $b$, para todo $a$ e $b$ em $X$. Em outras palavras, $\Upsilon(X)$ é o grupóide árvore sobre o conjunto de vértices $X$.

Definição 3.4.11. Dizemos que um espaço $X$ é contrátil a um ponto a, se existe uma aplicação contínua $F: X \times[0,1] \rightarrow X$ tal que

$$
\left.\begin{array}{l}
F(x, 0)=x \\
F(x, 1)=a
\end{array}\right\} \text { para todo } x \text { em } X
$$


Proposição 3.4.12. Seja $X$ um espaço e a um ponto em X. São equivalentes:

(i) X é contrátil a a.

(ii) A inclusão $\{a\} \rightarrow X$ e a aplicação trivial $X \rightarrow\{a\}$ são homotopicamente inversas.

(iii) X é homotopicamente equivalente a $\{a\}$.

Observação 3.4.13. Segue da proposição anterior, que um espaço topológico $X$ contrátil a um ponto a, é simplesmente conexo.

Definição 3.4.14. Sejam $X$ e $Y$ espaços topológicos. Uma aplicação $f: X \rightarrow Y$ é chamada um mergulho se $f: X \rightarrow f(X)$ é um homeomorfismo.

Definição 3.4.15. Sejam $X$ e $Y$ espaços topológicos. Sejam $\varphi, \psi: X \rightarrow Y$ mergulhos.

(i) Diremos que $\varphi$ e $\psi$ são isotópicos se existir um mergulho $F: X \times I \rightarrow Y \times I$ tal que

$$
F(x, t)=(f(x, t), t), \text { para } 0 \leq t \leq 1 \text { e } x \in X
$$

com $f(x, 0)=\varphi(x)$ e $f(x, 1)=\psi(x)$, para todo $x \in X . F$ é chamada isotopia entre $\varphi$ e $\psi$.

(ii) Seja $C$ um sub-espaço topológico de $X$. Diremos que $\varphi$ e $\psi$ são isotópicos relativamente a $C$ se existir uma isotopia $F$ entre $\varphi$ e $\psi$ tal que

$$
F(c, t)=\varphi(c)=\psi(c), \text { para todo } c \in C
$$

(iii) Diremos que $\varphi$ e $\psi$ são ambiente isotópicos se existir um mergulho $H: Y \times I \rightarrow Y \times I \mathrm{com}$

$$
H(y, t)=(h(y, t), t), \text { para } 0 \leq t \leq 1 \text { e } y \in Y
$$

satisfazendo as seguintes condições: 
(1) $\psi(x)=h(\varphi(x), 1)$, para todo $x \in X$

(2) $h(y, 0)=y$, para todo $y \in Y$.

$H$ é chamada isotopia ambiente.

Proposição 3.4.16. Isotopia de mergulhos de um espaço $X$ num espaço $Y$ é uma relação de equivalência.

\subsection{O Teorema de Seifert-Van Kampen}

Teorema 3.5.1 (Seifert-Van Kampen). Sejam $X_{1}$ e $X_{2}$ sub-espaços de um espaço $X$, e seja $X$ a união dos interiores de $X_{1}$ e $X_{2}$. Então, as inclusões induzem o diagrama push-out de grupóides

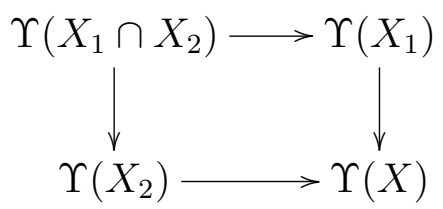

Usando o exemplo 2.4.9 do capítulo 2, obtemos o seguinte corolário.

Corolário 3.5.2. Com as mesmas hipóteses do teorema anterior, seja $V$ um subconjunto de $X$ tal que $V \cap X_{1}, V \cap X_{2}$ e $V \cap X_{1} \cap X_{2}$ intercepta cada componente conexa por caminho de $X_{1}, X_{2}$ e $X_{1} \cap X_{2}$, respectivamente. Então, as inclusões induzem um diagrama push-out de grupóides

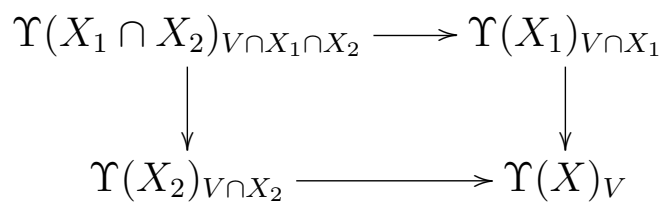

Corolário 3.5.3. Nas mesmas hipóteses do teorema 3.5.1, suponha $X_{1}, X_{2}$ e $X_{1} \cap X_{2}$ conexos por caminhos e considere um ponto a em $X_{1} \cap X_{2}$. Então as inclusões induzem um push-out de grupos

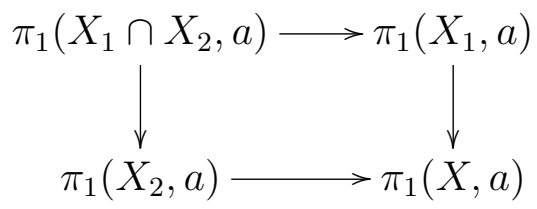


Exemplo 3.5.4. Seja $X=S^{1}, a=(1,0)$ e $b=(-1,0)$. Seja $X_{1}=S^{1} \backslash\{(0,-1)\}$ e $X_{2}=S^{1} \backslash\{(0,1)\}$. Não é possivel aplicar o corolário 3.5.3, pois $X_{1} \cap X_{2}$ não é conexo por caminhos, não obstante, $X_{1} \cap X_{2}$ consiste de duas componente conexas por caminhos contráteis, uma contendo a e a outra b. Além disso, $X_{1}$ e $X_{2}$ tambem são contráteis, portanto, $\Upsilon\left(X_{1}\right)_{\{a, b\}}$ e $\Upsilon\left(X_{2}\right)_{\{a, b\}}$ são grupóides árvore sobre o conjunto $\{a, b\}$, enquanto que $\Upsilon\left(X_{1} \cap X_{2}\right)_{\{a, b\}}$ é o grupóide discreto sobre $\{a, b\}$. Segue do corolário 3.5.2, que as inclusões induzem um diagrama push-out de grupóides

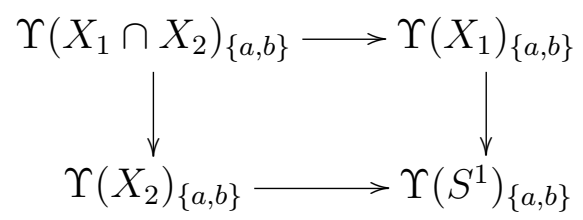

Finalmente, pelo exemplo 2.4.7 (ver demonstração), $\pi_{1}\left(S^{1}, a\right)=\langle t\rangle$, com $t$ de ordem infinita.

Observação 3.5.5. O homomorfismo induzido pela inclusão $A \hookrightarrow X$ não é sempre injetor.

Demonstração. De fato, é fácil ver que $\pi_{1}\left(D^{2}\right)=\{0\}$, portanto, a inclusão $S^{1} \hookrightarrow D^{2}$ induz o homomorfismo $\mathbb{Z} \rightarrow\{0\}$ o qual é claramente não injetor.

Definição 3.5.6. Seja A um subespaço de um espaço topológico X.

(i) A é chamado um retrato de $X$, se existe uma aplicação contínua $r: X \rightarrow A$ tal que $r(a)=a$, para todo a em A. A aplicação $r$ é chamada uma retração.

(ii) A é chamado retrato por deformação de $X$, se $i \circ r \simeq I d_{X}$, onde $i: A \hookrightarrow X$ é a inclusão, e tal homotopia é relativa ao conjunto A; isto é, existe uma homotopia $F: X \times[0,1] \rightarrow X$ entre $i$ or e $I_{X}$ tal que

$$
F(a, t)=a, \text { para todo } a \in A \text { e } t \in[0,1]
$$

Observação 3.5.7. Se A é um retrato de $X$ mediante uma retração $r$, então $r \circ i=I d_{A}$, onde $i: A \hookrightarrow X$ é a inclusão. 


\section{Proposição 3.5.8.}

(1) Se $A$ é um retrato de $X$ e a é um ponto de $A$, então a inclusão $i: A \hookrightarrow X$ induz um monomorfismo $i_{*}: \pi_{1}(A, a) \rightarrow \pi_{1}(X, a)$.

(2) Se A é um retrato por deformação de $X$ e a é um ponto de $A$, então a inclusão $i: A \hookrightarrow X$ induz um isomorfismo $i_{*}: \pi_{1}(A, a) \rightarrow \pi_{1}(X, a)$.

(3) Se $A$ é um retrato por deformação de $X$ então a inclusão $i: A \hookrightarrow X$ induz um isomorfismo $i_{*}: \Upsilon(A) \rightarrow \Upsilon(X)$.

Proposição 3.5.9. Seja $X_{1}$ e $X_{2}$ espaços qualquer. Seja A um retrato por deformação de $X_{2}$ e seja $f: A \rightarrow X_{1}$ uma aplicação contínua. Então $X_{1}$ é um retrato por deformação do espaço $X_{1} \cup_{f} X_{2}$.

Exemplo 3.5.10. Seja $X$ um espaço conexo por caminhos. Sejam $A_{1}$ e $A_{0}$ subespaços conexos por caminhos de $X$ tais que existe um homeomorfismo $\varphi: A_{0} \rightarrow A_{1}$. Considere o subespaço $A_{0} \times\{0,1\}$ de $A_{0} \times[0,1]$ e defina a função contínua $f: A_{0} \times\{0,1\} \rightarrow X$ por

$$
f(a, 0)=a \quad e \quad f(a, 1)=\varphi(a)
$$

Seja

$$
Y=X \cup_{f}\left(A_{0} \times[0,1]\right)
$$

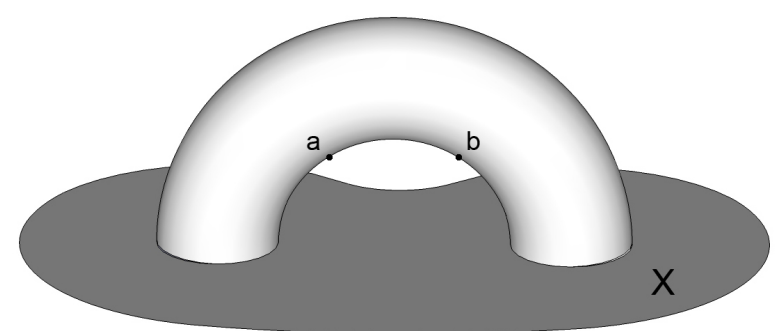

Figura 3.4: Espaço $Y$

Então,

$$
\pi_{1}(Y) \simeq \pi_{1}(X) *\langle t\rangle /\left\langle t^{-1} a t=\varphi_{*}(a): a \in \pi_{1}\left(A_{0}\right)\right\rangle^{N}
$$

com $t$ de ordem infinita e $N=\pi_{1}(X) *\langle t\rangle$. 
Demonstração. Considere

$$
Y_{1}=A_{0} \times(0,1) ; \quad Y_{2}=Y \backslash\left(A_{0} \times\left\{\frac{1}{2}\right\}\right) ; \quad Y_{0}=Y_{1} \cap Y_{2}
$$

Com as notações do exemplo 2.4.8 temos

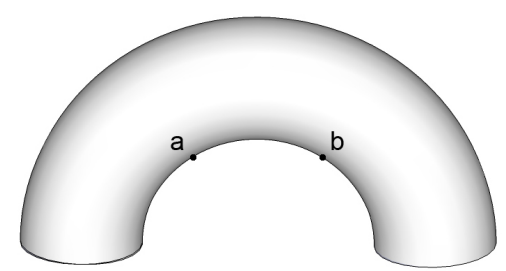

Figura 3.5: Espaço $Y_{1}$

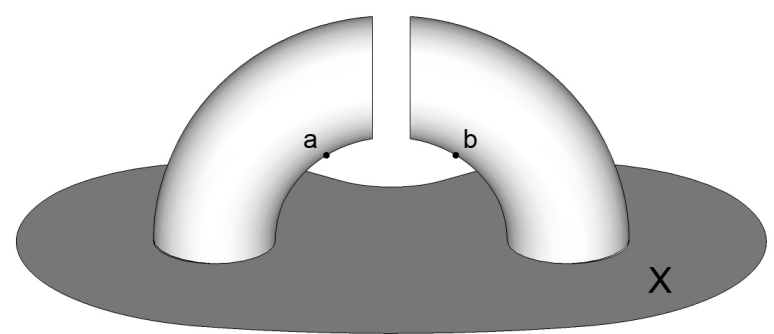

Figura 3.6: Espaço $Y_{2}$
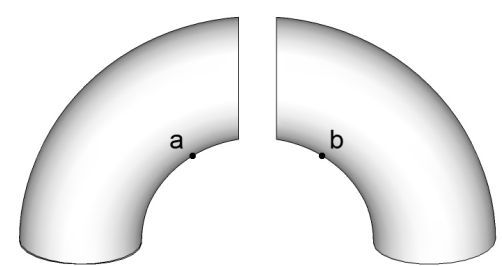

Figura 3.7: Espaço $Y_{0}$

(i) $a=\left(a_{0}, \frac{1}{3}\right)$ e $b=\left(a_{0}, \frac{2}{3}\right)$, com $a_{0}$ em $A_{0}$.

(ii) $K=\Upsilon\left(Y_{0}\right)_{\{a, b\}}, H=\Upsilon\left(Y_{1}\right)_{\{a, b\}}$ e $G=\Upsilon\left(Y_{2}\right)_{\{a, b\}}$

Observe que $A_{0} \cup \dot{U} A_{0}, A_{0}$ e $X$ são retratos por deformação de $Y_{0}, Y_{1}$ e $Y_{2}$, respectivamente. Portanto,

$$
\Upsilon\left(Y_{0}\right) \simeq \Upsilon\left(A_{0}\right) \times \Upsilon\left(A_{0}\right) ; \quad \Upsilon\left(Y_{1}\right) \simeq \Upsilon\left(A_{0}\right) ; \quad \Upsilon\left(Y_{2}\right) \simeq \Upsilon(X)
$$

Usando estas identificações temos que qualquer caminho em $Y_{1}$ de $a$ até $b$ é homotopicamente nulo em $A_{0}$. Também, um caminho em $Y_{2}$ de $a$ até $b$ é homotópico a um caminho 
$X$ de $a$ até $\varphi\left(a_{0}\right)$. Portanto, os homomorfismos $\alpha$ e $\beta^{\prime \prime}$ do exemplo 2.4.8 correspondem à identidade de $\pi_{1}\left(A_{0}\right)$ e $\varphi_{*}$, respectivamente. Portanto,

$$
\pi_{1}(Y) \simeq \pi_{1}(X) *\langle t\rangle /\left\langle t^{-1} a t=\varphi_{*}(a): a \in \pi_{1}\left(A_{0}\right)\right\rangle^{N}
$$

com $t$ de ordem infinita e $N=\pi_{1}(X) *\langle t\rangle$.

Um caso particular do exemplo anterior é o seguinte.

Exemplo 3.5.11. Seja $X$ um espaço conexo por caminhos e $\varphi: X \rightarrow X$ um homeomorfismo. Considere o espaço

$$
Y=X \cup_{f}(X \times[0,1])
$$

onde $f: X \times\{0,1\} \rightarrow X$ é definida por $f(x, 0)=x$ e $f(x, 1)=\varphi(x)$. Y também é conexo por caminhos. Então, pelo exemplo 3.5.10, temos (a extensão HNN)

$$
\pi_{1}(Y) \simeq \pi_{1}(X) *\langle t\rangle /\left\langle t^{-1} a t=\varphi_{*}(a): a \in \pi_{1}(X)\right\rangle^{N}
$$

com $t$ de ordem infinita e $N=\pi_{1}(X) *\langle t\rangle$

\subsection{Complexo CW}

Nesta seção vamos apresentar as definições e propriedades básicas dos espaços chamados complexos CW.

Definição 3.6.1. Um espaço $C$ é chamado n-célula, se é homemorfo ao interior do disco $n$-dimensional $D^{n}$. Vamos nos referir a um espaço por (simplesmente) célula, quando este for uma $n$-célula, para algum $n \geq 0$.

Proposição 3.6.2. Os conjuntos interiores de $D^{m}$ e $D^{n}$ são homeomorfos, se e somente se, $m=n$.

Definição 3.6.3. Dada uma n-célula, vamos chamar ao número $n$ de dimensão da célula.

Pela proposição 3.6.2, a dimensão de uma célula está bem definida. 
Definição 3.6.4. Uma decomposição em células de um espaço X, é uma família

$$
\Im=\left\{e_{\alpha}: e_{\alpha} e ́ \text { uma célula, } \alpha \in \Lambda\right\}
$$

tal que

$$
X=\bigcup_{\alpha \in \Lambda}^{\cdot} e_{\alpha}
$$

O n-esqueleto de $X$ associado à decomposição em células $\Im$, é o subespaço

$$
X^{n}=\bigcup_{\substack{\alpha \in \Lambda \\ \operatorname{dim}\left(e_{\alpha}\right) \leq n}} e_{\alpha}
$$

Definição 3.6.5. Seja $X$ um espaço topólogico de Hausdorff e $\Im$ uma decomposição em células de $X$. O par $(X, \Im)$ é chamado complexo $\mathbf{C W}$, se as seguintes condições estão satisfeitas:

(1) Para cada n-célula $e_{\alpha}$ em $\Im$, existe uma aplicação contínua $\phi_{e_{\alpha}}: D^{n} \rightarrow X$ que aplica a fronteira $S^{n-1}$ em $X^{n-1}$ e tal que sua restrição ao interior de $D^{n}$ é um homeomorfismo cuja imagem é $e_{\alpha}$.

(2) Para toda célula $e_{\alpha}$ em $\Im$, o fecho $\overline{e_{\alpha}}$ intecerpta um número finito de células em $\Im$.

(3) Um subconjunto $A$ de $X$ é fechado, se e somente se, $A \cap \overline{e_{\alpha}}$ é fechado em $X$, para cada célula e em $e_{\alpha}$ э.

Intuitivamente, um complexo CW é um espaço formado colando espaços homeomorfos a discos $n$ dimensionais. 


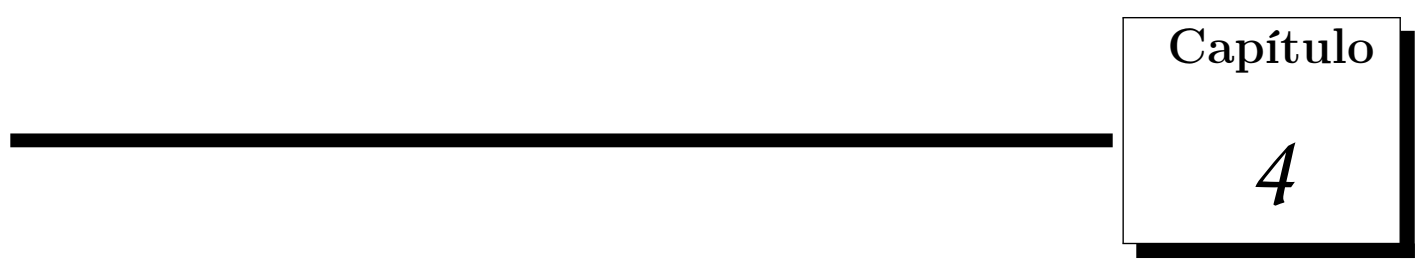

Tranças Geométricas de Artin e

\section{Enlaçamentos}

\subsection{Tranças Geométricas}

Seja $\mathbb{E}^{3}$ o espaço euclideano 3-dimensional. Identificamos $\mathbb{E}^{3}$ com $\mathbb{R}^{3}$ escolhendo um sistema de coordenadas $(x, y, z)$ no qual o eixo $Z$ aponta verticalmente para baixo (ver figura 4.1). Considere dois planos paralelos em $\mathbb{E}^{3}$ com a $z$-ésima coordenada constante, $z=z_{0}$ e $z=z_{1}$, com $z_{0}<z_{1}$. Chamaremos ao plano $z=z_{0}$ plano superior e ao plano $z=z_{1}$ plano inferior. Considere $n$ pontos $P_{1}, \ldots, P_{n}$ sobre uma reta no plano superior e projete-os ortogonalmente sobre o plano inferior, obtendo os pontos $P_{1}^{\prime}, \ldots, P_{n}^{\prime}$.

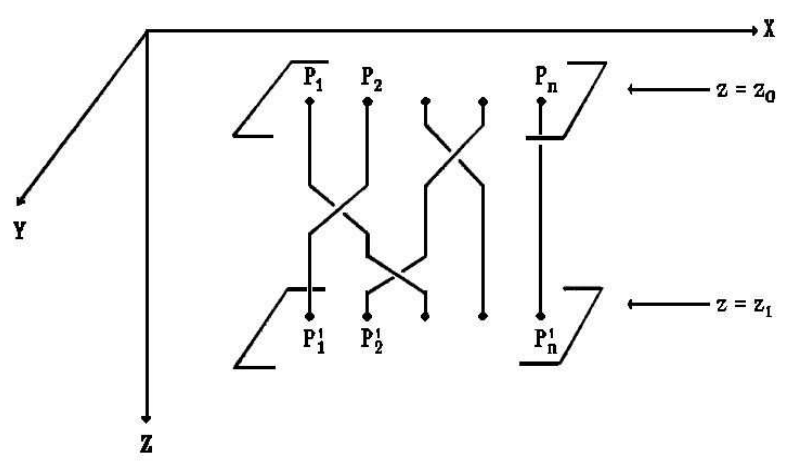

Figura 4.1: Uma trança geométrica em $\mathbb{E}^{3}$.

Definição 4.1.1. Uma trança geométrica de n-cordas (ou, n-trança) $\beta$ é um sistema 
de arcos mergulhados $\mathscr{A}=\left\{\mathscr{A}_{1}, \ldots, \mathscr{A}_{n}\right\}$ em $\mathbb{E}^{3}$, onde o $i$-ésimo arco $\mathscr{A}_{i}$ conecta o ponto $P_{i}$ do plano superior ao ponto $P_{\tau(i)}^{\prime}$ no plano inferior, para alguma permutação $\tau$ do conjunto $\{1, \ldots, n\}$, satisfazendo as seguintes condições:

(i) Cada arco $\mathscr{A}_{i}$ intercepta cada plano paralelo intermediário entre os planos superior e inferior exatamente uma vez;

(ii) Os arcos $\left\{\mathscr{A}_{1}, \ldots, \mathscr{A}_{n}\right\}$ interceptam cada plano paralelo intermediário entre os planos superior e inferior em exatamente $n$ pontos distintos.

A permutação $\tau$ é chamada permutação da trança. O arco $\mathscr{A}_{i}$ é chamado de $i$-ésima corda da trança.

Podemos pensar num arco em $\mathbb{E}^{3}$ como a imagem de um mergulho $\mathscr{A}_{i}:[0,1] \rightarrow \mathbb{E}^{3}$, onde $[0,1]$ é o intervalo unitario. Usaremos a mesma notação para o arco e o mergulho correspondente.

É natural perguntar-se quando uma trança pode ser deformada em outra continuamente. Isto motiva a seguinte definição.

Definição 4.1.2. Duas $n$-tranças $\mathscr{A}^{0}=\left\{\mathscr{A}_{1}^{0}, \ldots, \mathscr{A}_{n}^{0}\right\}$ e $\mathscr{A}^{1}=\left\{\mathscr{A}_{1}^{1}, \ldots, \mathscr{A}_{n}^{1}\right\}$ com a mesma permutação $\tau$ são chamadas equivalentes, se existir uma homotopia através de tranças geométricas com permutação $\tau$ de $\mathscr{A}^{0}$ a $\mathscr{A}^{1}$, em outras palavras, se existirem $n$ aplicações continuas

$$
F_{i}:[0,1] \times[0,1] \rightarrow \mathbb{E}^{3}, 1 \leq i \leq n
$$

satisfazendo

$$
\left.\begin{array}{l}
F_{i}(t, 0)=\mathscr{A}_{i}^{0}(t) \\
F_{i}(t, 1)=\mathscr{A}_{i}^{1}(t)
\end{array}\right\} 0 \leq t \leq 1,1 \leq i \leq n
$$

e

$$
\left.\begin{array}{l}
F_{i}(0, s)=P_{i} \\
F_{i}(1, s)=P_{\tau(i)}^{\prime}
\end{array}\right\} 0 \leq s \leq 1,1 \leq i \leq n
$$

e tal que se definirmos $\mathscr{A}_{i}^{s}:[0,1] \rightarrow \mathbb{E}^{3}$ por $\mathscr{A}_{i}^{s}(t)=F_{i}(t, s)$, então $\mathscr{A}^{s}=\left\{\mathscr{A}_{1}^{s}, \ldots, \mathscr{A}_{n}^{s}\right\}$ é uma n-trança geométrica (com permutação $\tau$ ), para cada $0 \leq s \leq 1$.

Observação 4.1.3. Usaremos a mesma notação para uma trança e sua classe de equivalência. 
Usando homotopia, se é necessario, podemos assumir que uma trança $\beta$ consiste só de arcos poligonais e que os cruzamentos entre os arcos são transversais quando projetamos a trança sobre o plano em $\mathbb{E}^{3}$ contendo os pontos $P_{1}, \ldots, P_{n}, P_{1}^{\prime}, \ldots, P_{n}^{\prime}$. Uma tal projeção será chamada projeção padrão da $n$-trança $\beta$ como se mostra na figura 4.2. Também podemos supor que o cruzamento dos arcos acontece em diferentes níveis e indicar quais cordas estão por cima e quais estão por baixo nos cruzamentos.

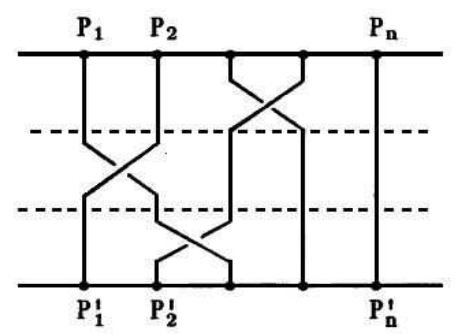

Figura 4.2: Projeção padrão de uma 5-trança

A figura 4.2 mostra um exemplo de como uma trança pode ser decomposta em tranças elementares definidas a seguir.

Definição 4.1.4. Para $1 \leq i \leq n-1$, vamos denotar por $\sigma_{i}$ a $n$-trança geométrica elementar, na qual a $i$-ésima corda cruza por cima a $(i+1)$-ésima corda uma única vez e todas as outras cordas vão do começo ao fim sem se cruzar.

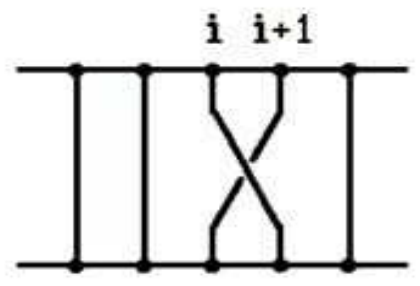

Figura 4.3: $i$-ésima trança elementar em $B_{n}$.

Seja $B_{n}$ o conjunto das classes de equivalência de todas as tranças geométricas com $n$-cordas. Vamos dotar $B_{n}$ de uma estrutura de grupo definindo uma multiplicação entre as $n$-tranças.

Sejam $\beta_{1}$ e $\beta_{2}$ duas $n$-tranças. Definimos o produto (composição) de $\beta_{1} \operatorname{com} \beta_{2}$, denotado por $\beta_{1} \cdot \beta_{2}$, como segue: primeiro, cole o plano inferior de $\beta_{1}$ com o plano superior de $\beta_{2}$ através dos $n$ pontos de cada plano. Tire o plano mediante o qual as 

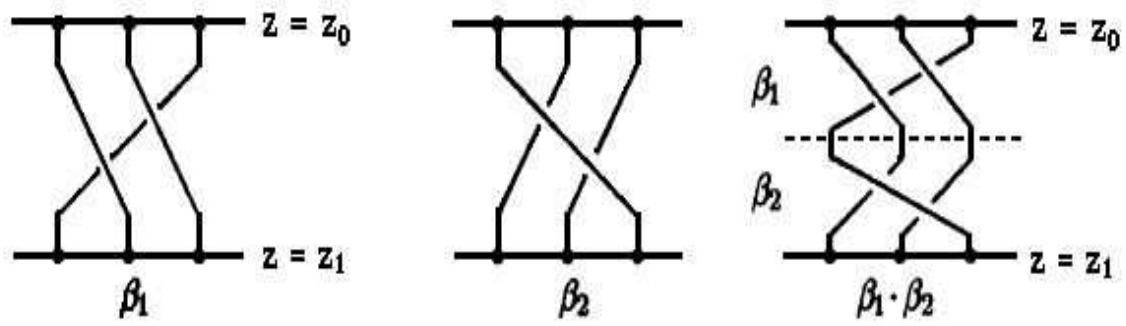

Figura 4.4: O produto de duas tranças.

tranças foram coladas e então comprima o sistema de arcos resultante até ficar entre os planos $z=z_{0}$ e $z=z_{1}$, obtendo assim a $n$-trança $\beta_{1} \cdot \beta_{2}$ (veja figura 4.4).

Vamos demonstrar que o produto induzido por $\cdot$ em $B_{n}$ é um produto bem definido. Sejam $\beta_{1}, \beta_{2}, \beta_{1}^{\prime}$ e $\beta_{2}^{\prime} n$-tranças geométricas tais que $\beta_{1}$ é equivalente $\beta_{1}^{\prime}$ e $\beta_{2}$ é equivalente a $\beta_{2}^{\prime}$. Afirmamos que o produto $\beta_{1} \cdot \beta_{2}$ é equivalente ao produto $\beta_{1}^{\prime} \cdot \beta_{2}^{\prime}$. De fato, seja $\tau$ a permutação associada a $\beta_{1}$ (e portanto, a $\beta_{1}^{\prime}$ ) e sejam $F_{i}$ e $G_{i}$, para $0 \leq i \leq n$, as homotopias requeridas entre os sistemas de cordas de $\beta_{1}$ e $\beta_{1}^{\prime}$, e de $\beta_{2}$ e $\beta_{2}^{\prime}$, respectivamente. Então, é claro que para $i=1, \ldots, n$, a aplicação $H_{i}: I \times I \rightarrow \mathbb{E}^{3}$ definida por

$$
H_{i}(t, s)= \begin{cases}F_{i}(2 t, s), & 0 \leq t \leq \frac{1}{2}, \quad s \in I \\ G_{\tau(i)}(2 t-1, s), & \frac{1}{2} \leq t \leq 1, \quad s \in I\end{cases}
$$

é parte das homotopias requeridas entre o sistema de cordas de $\beta_{1} \cdot \beta_{2}$ e $\beta_{1}^{\prime} \cdot \beta_{2}^{\prime}$. Portanto, o produto $\cdot$ em $B_{n}$ está bem definido.

A n-trança trivial, denotada por $\epsilon$, é aquela $n$-trança na qual todas as cordas vão diretamente do plano superior em linha reta para o plano inferior sem cruzamentos. A projeção padrão de $\epsilon$ se mostra na figura 4.5. É fácil ver que a classe de equivalência de $\epsilon$ é o elemento neutro para o produto induzido em $B_{n}$.

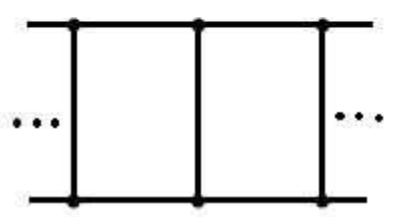

Figura 4.5: $n$-trança trivial.

A trança inversa de uma trança $\beta$, denotada por $\beta^{-1}$, é a imagem refletida num espelho da trança $\beta$, com respeito a um plano horizontal entre o plano superior e inferior, como mostra a figura 4.6 . 


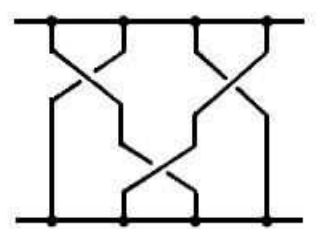

$\beta$

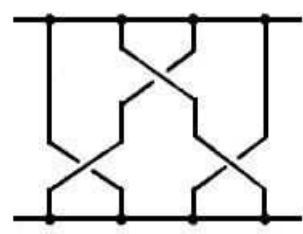

$\beta^{-1}$

Figura 4.6: A $n$-trança inversa.

É claro que a classe de equivalência de $\beta^{-1}$ está bem definida. De fato, suponha que $F_{i}$, para $i=1, \ldots n$, compõem as homotopias requeridas entre os sistemas de cordas de duas $n$-tranças equivalentes dadas, com permutação associada $\tau$. Então, para $i=1, \ldots, n$ a aplicação $G_{i}: I \times I \rightarrow \mathbb{E}^{3}$ definida por

$$
G_{i}(t, s)=F_{\tau(i)}(1-t, s)
$$

compõe o conjunto de homotopias requeridas entre os sistemas de cordas das $n$-tranças inversas das $n$-tranças dadas. Além disso, as tranças produto $\beta \cdot \beta^{-1}$ e $\beta^{-1} \cdot \beta$ são homotópicas à trança trivial de $n$-cordas. Portanto, a classe de equivalência de $\beta^{-1}$ é o elemento inverso em $B_{n}$ da classe de equivalência da trança $\beta$.

Para uma $n$-trança elementar $\sigma_{i}$, com $1 \leq i \leq n-1$, a trança inversa $\sigma_{i}^{-1}$ é obtida fazendo-se com que a $i$-ésima corda passe por baixo da $(i+1)$-ésima corda em vez de por cima (veja figura 4.7).
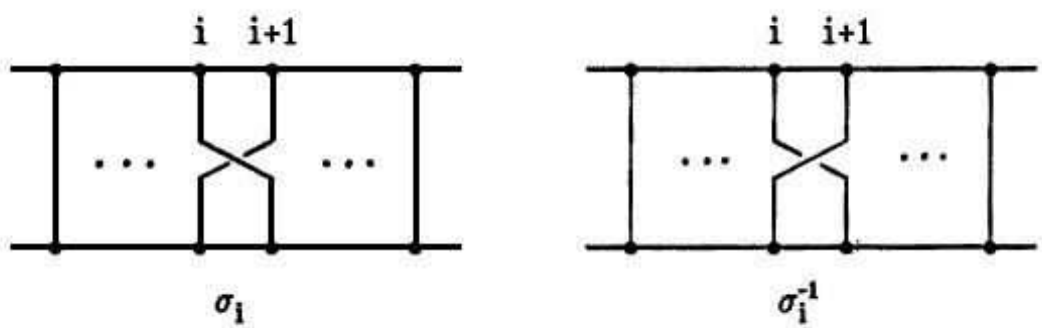

Figura 4.7: As $n$-tranças $\sigma_{i}$ e $\sigma_{i}^{-1}$.

Após as considerações acima, é fácil ver que $\left(B_{n}, \cdot\right)$ tem estrutura de grupo. Este grupo será chamado grupo de tranças de Artin com n-cordas, ou simplesmente, grupo de tranças de n-cordas.

A figura 4.2 mostra uma 5-trança escrita como produto das tranças elementares $\sigma_{i}$ e suas inversas (mais exatamente $\sigma_{3}^{-1} \sigma_{1}^{-1} \sigma_{2}$ ).

É intuitivo que toda (classe de equivalência de uma) n-trança pode ser escrita como 
produto de $n$-tranças elementares $\sigma_{i}$, para $0<i \leq n-1$, e suas $n$-tranças inversas $\sigma_{i}^{-1}$. Em outras palavras, as tranças elementares geram o grupo $B_{n}$.

Observemos algumas relações entre os elementos de $B_{n}$ :

- Se $|i-j|>1$ então a $i$-ésima e $(i+1)$-ésima corda não interferem com a $j$-ésima e $(j+1)$-ésima corda. Então, para $i, j \in\{1, \ldots, n-1\}$ temos a seguinte relação em $B_{n}$ :

$$
\sigma_{i} \cdot \sigma_{j}=\sigma_{j} \cdot \sigma_{i},|i-j|>1
$$
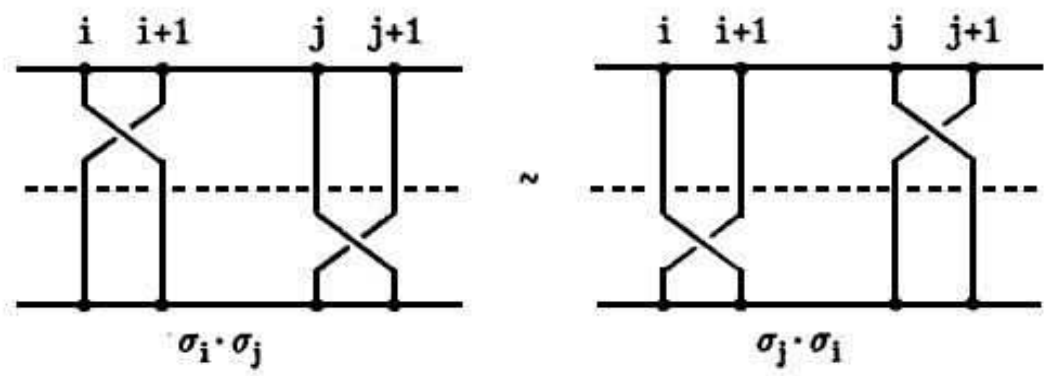

Figura 4.8: Relacão em $B_{n}$.

- Observamos também que

$$
\sigma_{i} \cdot \sigma_{i+1} \cdot \sigma_{i}=\sigma_{i+1} \cdot \sigma_{i} \cdot \sigma_{i+1}, 1 \leq i \leq n-2
$$
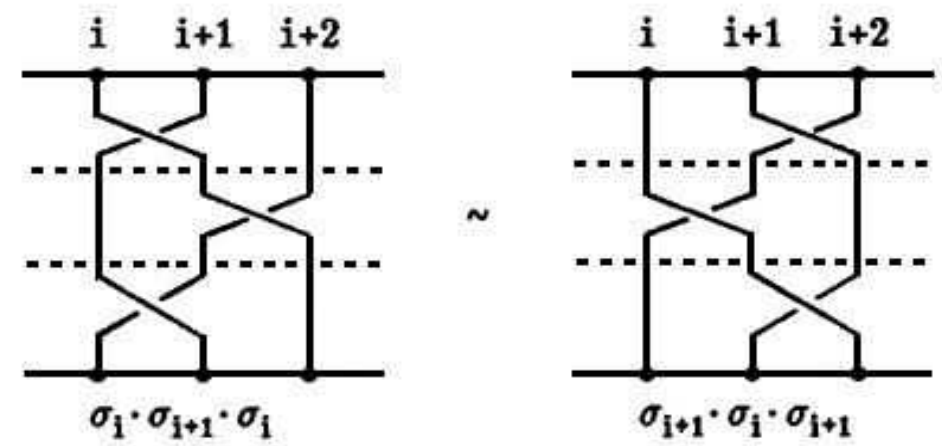

Figura 4.9: Relação em $B_{n}$.

Artin, no seu primeiro artigo sobre tranças de 1925, demonstrou que as relações (4.1.5) e (4.1.6) geram todas as relações entre os elementos de $B_{n}$. Isto não é trivial. A seguir enunciamos o teorema de Artin, chamado Teorema da Apresentação de Artin para o grupo Trança $B_{n}$. 
Teorema 4.1.5 (Da apresentação de Artin). O grupo $B_{n}$ das tranças geométricas com $n$-cordas admite uma apresentação com geradores $\sigma_{1}, \ldots, \sigma_{n-1}$ e relações

(1) $\sigma_{i} \cdot \sigma_{j}=\sigma_{j} \cdot \sigma_{i}$ para $|i-j|>1$, com $i, j=1, \ldots, n-1$

(2) $\sigma_{i} \cdot \sigma_{i+1} \cdot \sigma_{i}=\sigma_{i+1} \cdot \sigma_{i} \cdot \sigma_{i+1} \quad$ para $1 \leq i \leq n-2$

\subsection{Representação de Tranças por Automorfismos de Grupos Livres}

O teorema a seguir foi provado por Artin em 1925 e foi um dos teoremas que deu inicio à teoria de representação de tranças.

Teorema 4.2.1 (Da Representação de Artin). Seja $\mathbb{F}_{n}$ um grupo livre com $n$ geradores $x_{1}, \ldots, x_{n}, n \geq 1$ fixo. Denote por $\operatorname{Aut}\left(\mathbb{F}_{n}\right)$ o conjunto dos automorfismos (à direita) de $\mathbb{F}_{n}$. Então $B_{n}$ é isomorfo a um subgrupo de Aut $\left(\mathbb{F}_{n}\right)$ o qual consiste de todos os automorfismos $\bar{\beta}$ de $\mathbb{F}_{n}$ tais que

(1) $\left(x_{i}\right) \bar{\beta}=A_{i} x_{\tau(i)} A_{1}^{-1}$, para $1 \leq i \leq n$

(2) $\left(x_{1} \ldots x_{n}\right) \bar{\beta}=x_{1} \ldots x_{n}$

onde $\tau$ é uma permutação em $\Sigma_{n}$ e cada $A_{i}$ é um elemento em $\mathbb{F}_{n}$.

Além disso, sob este isomorfismo a trança elementar $\sigma_{i}$, para $1 \leq i \leq n-1$, é associada ao automorfismo (⿳亠口冋 direita) $\overline{\sigma_{i}}$ de $\mathbb{F}_{n}$ definido por

$$
\overline{\sigma_{i}}:\left\{\begin{array}{lll}
x_{i} & \longmapsto & x_{i} x_{i+1} x_{i}^{-1} \\
x_{i+1} & \longmapsto x_{i} \\
x_{j} & \longmapsto & x_{j} \quad, \text { para todo } j \neq i
\end{array}\right.
$$

Para demonstrar este teorema se usa o seguinte lema.

Lema 4.2.2. Suponha que $\xi$ seja um endormofismo (à direita) do grupo livre $\mathbb{F}_{n}$ com $n$ geradores $x_{1}, \ldots, x_{n}$ tal que 
(i) $\left(x_{i}\right) \xi=A_{i} x_{\tau(i)} A_{1}^{-1}$ para $1 \leq i \leq n$

(ii) $\left(x_{1} \ldots x_{n}\right) \xi=x_{1} \ldots x_{n}$

para alguma permutação $\tau$ em $\Sigma_{n}$ elementos $A_{i}$ em $\mathbb{F}_{n}$, para $1 \leq i \leq n$. Então, existe uma única (classe de equivalência de uma) n-trança geométrica $\beta$ em $B_{n}$ tal que $\xi=\bar{\beta}$. Em particular, $\xi$ é um automorfismo de $\mathbb{F}_{n}$.

Observação 4.2.3. O teorema 4.2.1 soluciona o problema de palavra em $B_{n}$, isto é, fornece um algoritmo para decidir quando um elemento em $B_{n}$ representa o elemento identidade. Equivalentemente, obtemos um algoritmo para decidir quando duas tranças são equivalentes.

Usando o teorema anterior podemos dar uma nova interpretação geométrica do grupo de tranças $B_{n}$. Considere o disco unitario $D^{2}$ no plano $\mathbb{E}^{2}$. A fronteira de $D^{2}$ é a circunferência unitaria $S^{1}$. Considere um conjunto de $n$ pontos distintos e fixos $Q_{n}=\left\{q_{1}, \ldots, q_{n}\right\}$ no interior de $D^{2}$. Escolha um ponto base $q_{0}$ em $S_{1}$. Então o grupo livre em $n$ geradores $x_{1}, \ldots, x_{n}$ pode ser identificado com o grupo fundamental de $D^{2} \backslash Q_{n}$, isto é,

$$
\mathbb{F}_{n}=\pi_{1}\left(D^{2} \backslash Q_{n}, q_{0}\right)
$$

onde cada gerador $x_{i}$ é representado por um laço em $D^{2}$ baseado em $q_{0}$ o qual circula $q_{i}$ no sentido anti-horário e evita os pontos $q_{j}$, para $j \neq i$, como mostra a figura 4.10.

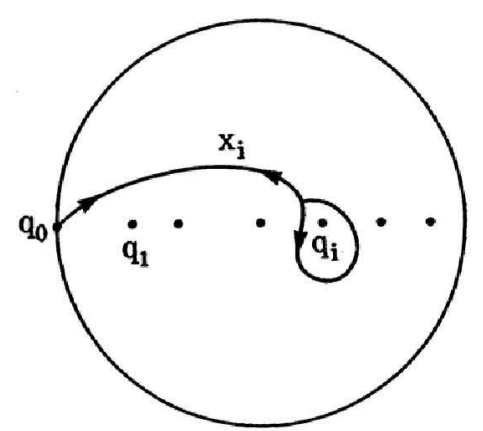

Figura 4.10: Laço representando o gerador $x_{i}$.

Seja $\mathscr{B}_{n}\left(D^{2}, S^{1}\right)$ o espaço dos homeomorfismos $h: D^{2} \rightarrow D^{2}$ satisfazendo as seguintes condições:

(1) Fixam $S^{1}$ ponto a ponto, isto é, $h(p)=p$, para todo $p$ em $S^{1}$. 


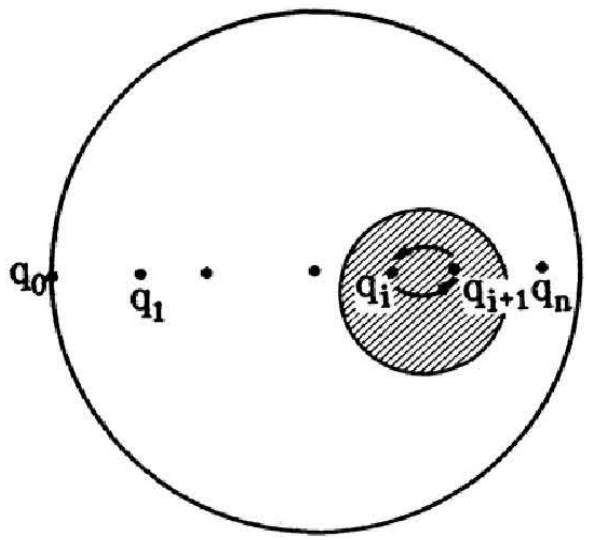

Figura 4.11: O homeomorfismo $h$.

(2) Permutam os pontos de $Q_{n}$ entre si, isto é, existe uma permutação $\tau$ em $\Sigma_{n}$ tal que $h\left(q_{i}\right)=q_{\tau(i)}$, para $i=1, \ldots, n$.

$\mathscr{B}_{n}\left(D^{2}, S^{1}\right)$ está dotado com a topologia compacto-aberta. Cada homeomorfismo $h$ : $D^{2} \rightarrow D^{2}$ em $\mathscr{B}_{n}\left(D^{2}, S^{1}\right)$ induz um automorfismo $h_{*}$ de $\pi_{1}\left(D^{2} \backslash Q_{n}, q_{0}\right)$, ou com a identificação feita, um automorfismo de $\mathbb{F}_{n}$.

Teorema 4.2.4. $O$ grupo de tranças $B_{n}$ pode ser identificado com o grupo dos automorfismos de $\mathbb{F}_{n}=\pi_{1}\left(D^{2} \backslash Q_{n}, q_{0}\right)$ induzidos pelos homeomorfismos $h \in \mathscr{B}_{n}\left(D^{2}, S^{1}\right)$.

Com este teorema e outro teorema clássico de Alexander de 1923 (conhecido por o Truque de Alexander) é possivel construir, de uma maneira diferente, uma $n$-trança geométrica.

Teorema 4.2.5 (O truque de Alexander). Seja $D^{n}$ a bola unitária no espaço euclideano $n$-dimensional $\mathbb{E}^{n}$ e seja a $n$-1-esfera $S^{n-1}=\partial\left(D^{n}\right)$ sua fronteira. Então todo homeomorfismo $h: D^{n} \rightarrow D^{n}$, que fixa $S^{n-1}$ ponto a ponto, é isotópico à aplicação identidade de $D^{n}$, sob uma isotopia que fixa $S^{n-1}$ ponto a ponto. Se $h(0)=0$, então a isotopia pode ser escolhida fixando $0 \mathrm{em} D^{n}$.

Seja $h: D^{n} \rightarrow D^{n}$ um homeomorfismo em $\mathscr{B}_{n}\left(D^{2}, S^{1}\right)$. Pelo teorema anterior, existe uma aplicação contínua

$$
H: D^{2} \times I \longrightarrow D^{2} \times I
$$

satisfazendo as seguintes condições:

(i) Para todo $t$ em $I, H$ aplica o nível $D^{2} \times\{t\}$ homeomorficamente sobre si mesmo; 
(ii) Os subespaços $D^{2} \times\{0\}$ e $S^{1} \times I$ são fixados ponto a ponto;

(iii) $\left.H\right|_{D^{2} \times\{1\}}$ define o homeomorfismo dado $h$ em $\mathscr{B}_{n}\left(D^{2}, S^{1}\right)$.

A imagem de $Q_{n} \times I$ sob $H$ é uma $n$-trança geométrica de $B_{n}$.

\subsection{Construindo Enlaçamentos a partir de Tranças}

Seja $\beta$ uma trança geométrica em $\mathbb{E}^{3}$. Colocamos a projeção padrão da trança $\beta$ no plano $\mathbb{E}^{2}$ de forma horizontal.

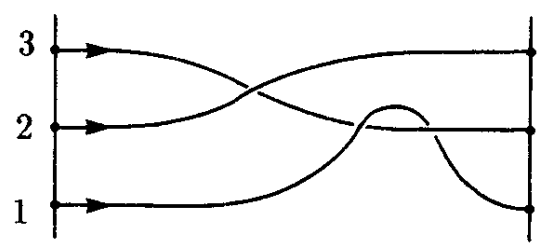

Figura 4.12: Projeção de uma 3-trança $\beta=\sigma_{2} \sigma_{1}^{2}$.

Seja $l$ um eixo vertical colocado atrás da trança $\beta$ nesta nova projeção. Feche a trança $\beta$ ao redor do eixo $l$ identificando os pontos iniciais e finais da trança $\beta$. O resultado é chamado fecho da trança $\beta$.

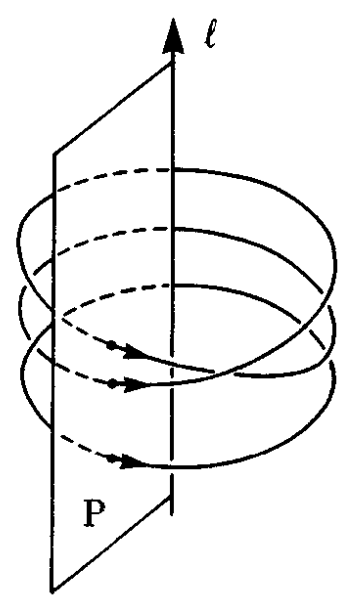

Figura 4.13: O fecho da trança $\beta$.

Dotamos às cordas da $n$-trança $\beta$ de uma orientação tal que na projeção da trança o sentido é de esquerda a direita. Esta orientação das cordas induz uma orientação no fecho da trança $\beta$. Também dotamos o eixo $l$ de uma orientação tal que quando um ponto se movimenta pelo fecho da trança em direção positiva, este gira ao redor do eixo $l$ segundo a regra da mão direita. Reciprocamente, se tomarmos o fecho da trança $\beta$ e fazermos um 
corte ao longo de um semi-plano $P$ determinado por $l$ e esticamos o resultado, obtemos a $n$-trança $\beta$.

Definição 4.3.1. Seja $m$ um inteiro positivo. Um enlaçamento (ou link) de $m$ componentes $V$ em $\mathbb{E}^{3}$ é a união de $m$ curvas poligonais, fechadas, simples, mutuamente disjuntas. $V$ também será chamado m-enlaçamento. Quando $m=1, V$ é chamado nó. Cada curva poligonal fechada simples disjunta das outras curvas é chamada componente de $V$.

Definição 4.3.2. Os segmentos de reta do enlaçamento $V$ são chamados arestas $e$ seus pontos inicial e final são chamados vértices. Usaremos a notação [ab] para denotar a aresta do enlaçamento $V$ com vértices a e b,

Seja $V$ um enlaçamento em $\mathbb{E}^{3}$ e seja $[a b]$ uma aresta de $V$. Seja $c$ um ponto em $\mathbb{E}^{3}$ diferente de $a$ e de $b$. Denote por $\triangle(a, b, c)$ o triângulo possivelmente degenerado com vértices $a, b$ e $c$. Suponha que $\triangle(a, b, c)$ intercepta o enlaçamento $V$ exatamente na aresta $[a b]$, isto é,

$$
V \cap \triangle(a, b, c)=[a b]
$$

Neste caso, dizemos que a deformação elementar $\mathscr{E}=\mathscr{E}_{a b}^{c}$ é aplicável a $V$, e construimos um novo enlaçamento, denotado por $\mathscr{E}_{a b}^{c} V$, e definido por

$$
\mathscr{E}_{a b}^{c}=(V \backslash[a b]) \cup[a c] \cup[c b]
$$

Em outras palavras, obtemos $\mathscr{E}_{a b}^{c} V$ por uma deformação de $V$ ao longo de um triângulo $\triangle(a, b, c)$ removendo $[a b]$ e substituindo-o pelas arestas $[a c]$ e $[c b]$.

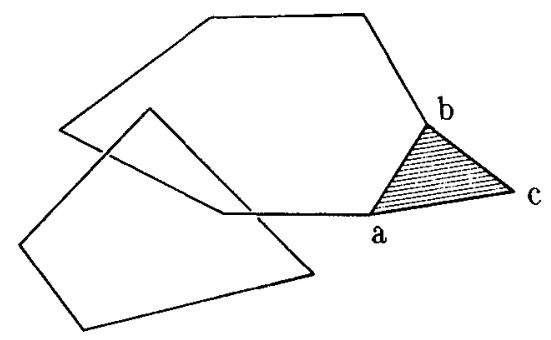

Figura 4.14: O enlaçamento $\mathscr{E}_{a b}^{c} V$.

Reciprocamente, se $[a c]$ e $[c b]$ são arestas consecutivas num enlaçamento $V^{\prime}$ em $\mathbb{E}^{3}$ tal que

$$
V^{\prime} \cap \triangle(a, b, c)=[a c] \cup[c b]
$$


então podemos realizar a deformação elementar inversa, denotada por $\mathscr{E}^{-1}=\left(\mathscr{E}_{a b}^{c}\right)^{-1}$, sobre $V^{\prime}$, substituindo as arestas $[a c]$ e $[c b]$ por $[a b]$

Como já foi indicado, nos referiremos a ambos $\mathscr{E}_{a b}^{c}$ e sua inversa $\left(\mathscr{E}_{a b}^{c}\right)^{-1}$ como deformação elementar.

Definição 4.3.3. Dois enlaçamentos $V$ e $V^{\prime}$ em $\mathbb{E}^{3}$ são ditos serem combinatoriamente equivalentes se um deles pode ser transformado no outro mediante uma sequência finita de deformações elementares.

Observação 4.3.4. Dois enlaçamentos $V$ e $V^{\prime}$ em $\mathbb{E}^{3}$ são combinatoriamente equivalentes se, e somente se, são isotópicos ambiente em $\mathbb{E}^{3}$, isto é, existe uma família contínua de homeomorfismos $h_{t}: \mathbb{E}^{3} \rightarrow \mathbb{E}^{3}$ sobre $\mathbb{E}^{3}$, com $0 \leq t \leq 1$, tal que $h_{0}=I d_{\mathbb{E}^{3}}$ é a aplicação identidade e tal que $h_{1}(V)=V^{\prime}$.

Em outras palavras, a observação 4.3.4 diz que a equivalência de enlaçamentos em $\mathbb{E}^{3}$ corresponde à noção intuitiva de ser capaz de deformar um enlaçamento $V$ continuamente em outro enlaçamento $V^{\prime}$

\subsection{Representando um Enlaçamento pelo Fecho de uma}

\section{Trança}

O teorema principal apresentado nesta seção conecta a noção de enlaçamento com a de trança.

Considere um enlaçamento $V$ em $\mathbb{E}^{3}$. Seja $l$ uma reta fixa em $\mathbb{E}^{3}$, chamada eixo, que não intercepta o enlaçamento $V$.

Definição 4.4.1. O enlaçamento $V$ é dito estar em posição geral com respeito à reta $l$ se nenhuma das arestas de $V$ é coplanar com a reta $l$.

Lema 4.4.2. Todo enlaçamento é combinatoriamente equivalente a um enlaçamento em posição geral.

Observação 4.4.3. Pelo lema anterior, agora trabalharemos só com enlaçamentos em posição geral com respeito a um eixo $l$. 
Seja $V$ um enlaçamento em $\mathbb{E}^{3}$ dotado de uma orientação, isto é, cada componente de $V$ está orientada. Considere um eixo $l$ também orientado. Estas orientações nos permitem dividir as arestas de $V$ em positivas e negativas. Vamos chamar uma aresta $[a b]$ de positiva se fixando um ponto em $[a b]$, distinto de $a$ e $b$, o semi-plano $P$ determinado pelo eixo $l$ e o ponto fixo, gira em torno de $l$ satisfazendo a regra da mão direita quando o ponto fixo se movimenta ao longo de $[a b]$ no sentido da orientação do enlaçamento $V$. Neste caso escrevemos $[a b]>0$. Uma aresta $[a b]$ é chamada negativa, se o semi-plano $P$ gira em torno de $l$ segundo a regra da mão esquerda, quando o ponto fixo em $[a b]$ se movimenta no sentido da orientação do enlaçamento $V$. Neste caso escrevemos $[a b]<0$.

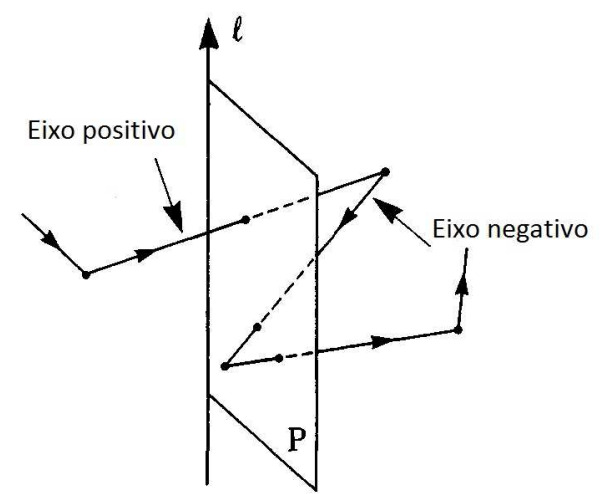

Figura 4.15: Eixo positivo e eixo negativo.

Definição 4.4.4. A altura de um enlaçamento $V$ em $\mathbb{E}^{3}$ é o número de arestas negativas em $V$. Este número será denotado por $h(V)$.

Lema 4.4.5. Um enlaçamento $V$ em $\mathbb{E}^{3}$ é o fecho de uma trança, se e somente se, admite uma orientação e um eixo l orientado tais que as arestas de $V$ sejam todas positivas.

Isto motiva a seguinte definição:

Definição 4.4.6. Uma trança fechada $\mathrm{em} \mathbb{E}^{3}$ é um enlaçamento orientado $V$ em $\mathbb{E}^{3}$, que admite um eixo orientado $l$ com respeito ao qual todas as arestas de $V$ são positivas.

Pelo lema 4.4.5 as tranças fechadas são exatamente os enlaçamentos em $\mathbb{E}^{3}$ que foram obtidos fechando tranças. Denotaremos por $\widehat{\beta}$ a trança fechada obtida da trança $\beta$ em $B_{n}$.

Teorema 4.4.7 (Alexander,1923). Todo enlaçamento $V$ em $\mathbb{E}^{3}$ é combinatoriamente equivalente a uma trança fechada. 


\subsection{Equivalência Combinatória de Tranças Fechadas}

Nesta seção vamos apresentar o teorema de Andrey Andreyevich Markov sobre equivalência combinatória de tranças fechadas. Este teorema foi apresentado por Markov em 1935, mas a primeira demonstração completa deste teorema não foi apresentada até o ano 1974 por Joan Birman, no seu livro "Braids, Links and Mappings Class Groups".

Seja $V$ um trança fechada (orientada) com eixo $l$ (orientado). Todas as arestas de $V$ são positivas com respeito à orientação de $l$. Vamos descrever dois tipos de deformação simples sobre as arestas positivas que pode ser aplicado a $V$.

\subsubsection{Uma deformação do tipo $\mathscr{R}$}

Suponha que $[a b]>0$ é uma aresta positiva em $V$, e seja $c$ um ponto de $\mathbb{E}^{3}$ tal que

$$
\triangle(a, b, c) \cap V=[a b]
$$

e $[a c]>0$ e $[c b]>0$ com respeito a $l$. Então a deformação elementar $\mathscr{E}_{a b}^{c}$ é aplicável a $V$. Em $V^{\prime}=\mathscr{E}_{a b}^{c} V$ a aresta $[a b]>0$ foi substituída por duas arestas, $[a c]>0$ e $[c b]>0$. A deformação $\mathscr{E}_{a b}^{c}$ e sua inversa são chamadas deformações do tipo $\mathscr{R}$.

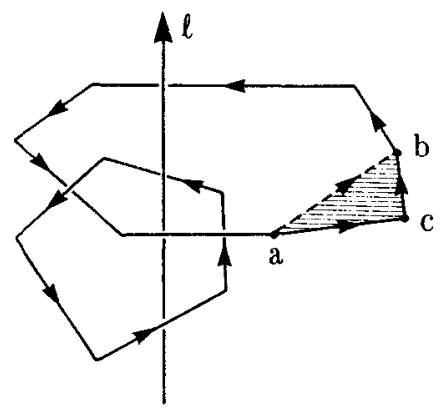

Figura 4.16: Deformação tipo $\mathscr{R}$.

Uma deformação do tipo $\mathscr{R}$ remove uma aresta positiva e a substitui por duas novas arestas positivas, ou viceversa.

\subsubsection{Uma deformação do tipo $\mathscr{W}$}

Suponha que $[a d]>0$ é uma aresta positiva em $V$, e seja $b$ um ponto em $\mathbb{E}^{3}$ tal que

$$
\triangle(a, d, b) \cap V=[a d]
$$


e tal que uma das arestas $[a b]$ ou $[b d]$ é positiva e a outra negativa com respeito ao eixo $l$. Então a deformação elementar $\mathscr{E}_{a b}^{c}$ é aplicável a $V$. Em $V^{\prime}=\mathscr{E}_{a b} c$, removemos a aresta positiva $[a d]$ de $V$ e a substituímos por duas novas arestas $[a b]$ e $[b d]$, uma positiva e a outra negativa com respeito ao eixo $l$. Suponha que $[b d]$ é negativa. Seja $c$ um ponto em $\mathbb{E}^{3}$ tal que

$$
\triangle(b, d, c) \cap V^{\prime}=[b d]
$$

e $[b c]>0$ e $[c d]>0$ com respeito ao eixo $l$. Então a deformação elementar $\mathscr{E}_{a b}^{c}$ é aplicável a $V^{\prime}$. Assim, combinando as duas deformações elementares $\mathscr{E}_{a d}^{b}$ e $\mathscr{E}_{b d}^{c}$, obtemos a trança fechada

$$
V^{\prime \prime}=\mathscr{E}_{b d}^{c} \mathscr{E}_{a d}^{b} V=(V \backslash[a d]) \cup[a b] \cup[b c] \cup[c d]
$$

na qual substituímos a aresta $[a d]$ em $V$ por três novas arestas positivas $[a b],[b c]$ e $[c d]$.

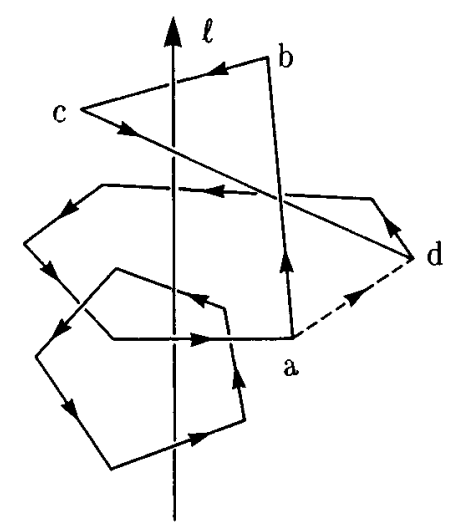

Figura 4.17: Deformação tipo $\mathscr{W}$ com $[b d]$ negativo.

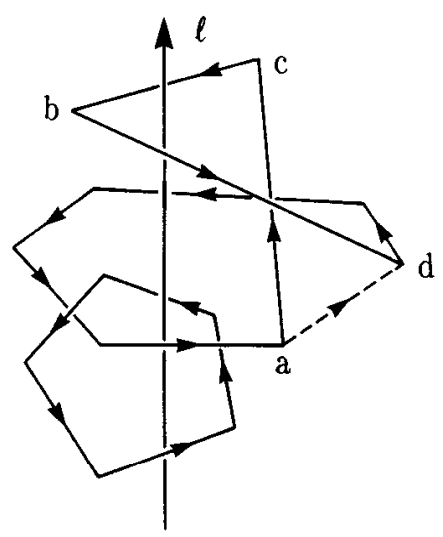

Figura 4.18: Deformação tipo $\mathscr{W}$ com $[b d]$ positivo.

Com estes dois tipos de movimentos a nossa diposição, vamos apresentar duas versões do teorema de Markov, a versão geométrica e a versão algébrica. 
Teorema 4.5.1 (Markov 1935: Versão Geométrica). Sejam $V$ e $V^{\prime}$ duas tranças fechadas em $\mathbb{E}^{3}$ combinatoriamente equivalentes. Então existe uma sequência finita de tranças fechadas em $\mathbb{E}^{3}$ :

$$
V=V_{0}, V_{1}, \ldots, V_{r-1}, V_{r}=V^{\prime}
$$

tal que para $0 \leq i<r, V_{i+1}$ é obtida de $V_{i}$ pela aplicação de uma deformação do tipo $\mathscr{R}$ ou do tipo $\mathscr{W}$. A recíproca é trivial.

Pelo teorema de Alexander (teorema 4.4.7) sabemos que todo enlaçamento em $\mathbb{E}^{3}$ é combinatoriamente equivalente a uma trança fechada, portanto, o teorema de Markov é um teorema sobre equivalência combinatória de dois enlaçamentos em $\mathbb{E}^{3}$ com generalidade completa.

Por outro lado, sabemos pelo lema 4.4.5 que tranças fechadas $V$ em $\mathbb{E}^{3}$ surgem pelo fechamento de uma trança aberta $\beta$ em $B_{n}$ em relação a um eixo adequado. Quando isto aconteçe, escrevemos $V=\widehat{\beta}$. A trança $\beta$ não é única pois para um enlaçamento qualquer existem várias tranças, com diferentes número de cordas, cujo fecho é o enlaçamento. Por isso escreveremos $(\beta, n)$ para denotar uma trança $\beta$ em $B_{n}$. Apresentamos a seguir, a versão algébrica do teorema de Markov.

Teorema 4.5.2 (Markov 1935: Versão algébrica). Sejam $\widehat{\beta}$ e $\widehat{\beta^{*}}$ duas tranças fechadas em $\mathbb{E}^{3}$, representadas pelas tranças $(\beta, n) e\left(\beta^{*}, n^{*}\right)$. Então $\widehat{\beta}$ é combinatoriamente equivalente a $\widehat{\beta^{*}}$ se, e somente se, existe uma sequência finita de movimentos

$$
(\beta, n)=\left(\beta_{0}, n_{0}\right) \rightarrow\left(\beta_{1}, n_{1}\right) \rightarrow \cdots \rightarrow\left(\beta_{r-1}, n_{r-1}\right) \rightarrow\left(\beta_{r}, n_{r}\right)=\left(\beta^{*}, n^{*}\right)
$$

transformando $(\beta, n)$ em $\left(\beta^{*}, n^{*}\right)$, tal que para $0 \leq i<r$, a trança $\left(\beta_{i+1}, n_{i+1}\right)$ pode ser obtida de sua antecessora $\left(\beta_{i}, n_{i}\right)$ por aplicação de um dos seguintes movimentos:

$\mathscr{M}_{1}$ : Substitua $\beta_{i}$ por qualquer outra trança em $B_{n_{i}}$ conjugada a $\beta_{i}$. Coloque $n_{i+1}=$ $n_{i}$.

$\mathscr{M}_{2}$ : Substitua $\left(\beta_{i}, n_{i}\right)$ por $\left(\beta_{i} \sigma_{n_{i}}^{ \pm 1}, n_{i}+1\right)$, ou, se $\beta_{i}=\gamma \sigma_{n_{i}-1}^{ \pm 1}$, onde a palavra correspondente a $\gamma$ envolve somente os geradores $\sigma_{1}, \ldots, \sigma_{n_{i}-2}$, substitua $\left(\beta_{i}, n_{i}\right)$ por $\left(\gamma, n_{i}-1\right)$ 
Este teorema é fundamental no estudo dos enlaçamentos, pois permite estudar um problema topológico de classificação de enlaçamentos, a partir do estudo de um problema algébrico relacionado com tranças. Para ser mais específico, considere o conjunto

$$
B_{\infty}=\left\{(\beta, n): \beta \in B_{n}, n \in \mathbb{N}\right\}
$$

$B_{\infty}$ denota a união disjunta de todos os grupos de tranças. Defina sobre $B_{\infty}$ a relação

$$
\begin{aligned}
(\beta, n) \sim\left(\beta^{*}, n^{*}\right) \Leftrightarrow & \text { existe uma sequência finita } \mathscr{M} \text { de movimentos } \\
& \text { de Markov tal que }\left(\beta_{1}, n_{1}\right) \stackrel{\mathscr{M}}{\longrightarrow}\left(\beta^{*}, n^{*}\right)
\end{aligned}
$$

é fácil ver que $\sim$ é uma relação de equivalência sobre $B_{\infty}$. Segue que as classes de equivalência das tranças sob $\sim$ podem ser identificadas com as classes de equivalência de enlaçamentos em $\mathbb{E}^{3}$ com respeito a equivalência combinatória.

\subsection{O Grupo de um Enlaçamento}

Seja $V$ um enlaçamento em $\mathbb{E}^{3}$. Mediante a compactificação por um ponto do espaço $\mathbb{E}^{3}$, obtemos a esfera 3-dimensional $S^{3}$. Podemos assim considerar o enlaçamento $V$ como um objeto em $S^{3}$ quando for conveniente. Como $V$ é limitado e unidimensional, segue que $\mathbb{E}^{3} \backslash V$ é conexo por caminhos e portanto, $S^{3} \backslash V$ também é conexo por caminhos e ambos espaços tem o mesmo grupo fundamental. De fato, seja $U$ uma vizinhança de $\infty$ em $S^{3}$ homeomorfa a $\mathbb{R}^{3}$ com $U \cap V=\emptyset$. Então

$$
U \cap \mathbb{R}^{3}=U \backslash\{\infty\} \simeq S^{2}
$$

Portanto, $U$ e $U \cap \mathbb{R}^{3}$ são simplesmente conexos. Como

$$
S^{3} \backslash V=U \cup\left(\mathbb{R}^{3} \backslash V\right)
$$

temos aplicando o teorema de Seifert-Van Kampen que $\pi_{1}\left(S^{3} \backslash V\right) \simeq \pi_{1}\left(\mathbb{R}^{3} \backslash V\right)$ (veja [6], pag. 51).

Definição 4.6.1. Dado um enlaçamento $V$ em $\mathbb{E}^{3}$, vamos chamar de grupo do enlaça- 
mento $V$ ao grupo fundamental do espaço $\mathbb{E}^{3} \backslash V$ (ou, do espaço $\left.S^{3} \backslash V\right)$. Vamos denotar

$$
G(V)=\pi_{1}\left(\mathbb{E}^{3} \backslash V\right)=\pi_{1}\left(S^{3} \backslash V\right)
$$

Observe que se dois enlaçamentos $V$ e $V^{\prime}$ em $\mathbb{E}^{3}$ são combinatoriamente equivalentes então seus complementares $\mathbb{E}^{3} \backslash V$ e $\mathbb{E}^{3} \backslash V^{\prime}$ são homeomorfos. Portanto, $G(V)$ e $G\left(V^{\prime}\right)$ são isomorfos. Temos portanto o

Lema 4.6.2. Enlaçamentos combinatoriamente equivalentes em $\mathbb{E}^{3}$ têm grupos isomorfos.

Observação 4.6.3. Pelo teorema de Alexander, todo enlaçamento em $\mathbb{E}^{3}$ é combinatoriamente equivalente a um enlaçamento $\widehat{\beta}$ obtido fechandose (ao redor de um eixo particular) uma trança $\beta$. Por isso, vámos estudar somente grupos de tranças fechadas.

Antes de apresentar o teorema fundamental desta seção, vamos introduzir algumas notações. Seja $\mathbb{F}_{n}$ um grupo livre com $n$-geradores $x_{1}, \ldots, x_{n}$, para um inteiro $n \geq 1$ fixo. Para um conjunto de elementos $A_{1}, \ldots, A_{n}$ em $\mathbb{F}_{n}$ e uma permutação $\tau$ em $\Sigma_{n}$, vamos denotar por $G\left(A_{1}, \ldots, A_{n} ; \tau\right)$ o grupo apresentado por

geradores: $x_{1}, \ldots, x_{n}$

relações definidoras: $x_{i}=A_{i} x_{\tau(i)} A_{i}^{-1}$ para $1 \leq i \leq n$

Teorema 4.6.4. Seja $\beta$ uma n-trança geométrica, e suponha que o automorfismo $\bar{\beta}$ de $\mathbb{F}_{n}$, associado a $\beta$, é dado por

$$
\left(x_{i}\right) \bar{\beta}=A_{i} x_{\tau(i)} A_{i}^{-1} \text { para } 1 \leq i \leq n
$$

como no teorema 4.2.1. Então o grupo $G(\widehat{\beta})$ do enlaçamento $\widehat{\beta}$ em $\mathbb{E}^{3}$ obtido fechando-se a trança $\beta$ é isomorfo ao grupo $G\left(A_{1}, \ldots, A_{n} ; \tau\right)$, e os elementos $A_{1}, \ldots, A_{n}$ em $\mathbb{F}_{n}$ e a permutação $\tau$ associada à trança $\beta$ satisfazem a identidade

$$
\left(A_{1} x_{\tau(1)} A_{1}^{-1}\right) \ldots\left(A_{n} x_{\tau(n)} A_{n}^{-1}\right)=x_{1} \ldots x_{n}
$$

Reciprocamente, se os elementos $A_{1}, \ldots, A_{n}$ em $\mathbb{F}_{n}$ e uma permutação $\tau$ em $\Sigma_{n}$ satisfazem 
a identidade (4.6.2), então existe uma n-trança geométrica $\beta$ para a qual

$$
G(\widehat{\beta})=G\left(A_{1}, \ldots, A_{n} ; \tau\right)
$$

Observe que uma das relações em $G(\widehat{\beta})$ do teorema anterior pode ser obtida a partir das outras. Daí o seguinte corolário.

Corolário 4.6.5. Seja $\beta$ em $B_{n}$ e seja $\widehat{\beta}$ a trança fechada associada a $\beta$. Então o grupo $G(\widehat{\beta})$ do enlaçamento $\widehat{\beta}$ admite a apresentação

$$
G(\widehat{\beta})=\left\langle x_{1}, \ldots, x_{n} ; x_{1}=\left(x_{1}\right) \bar{\beta}, \ldots, x_{n-1}=\left(x_{n-1}\right) \bar{\beta}\right\rangle
$$

onde $x_{1}, \ldots, x_{n}$ são os geradores do grupo livre $\mathbb{F}_{n}$ e $\bar{\beta}$ é o automorfismo de $\mathbb{F}_{n}$ é induzido por $\beta$.

\subsection{Algumas Definições da Teoria de Nós}

Nesta seção vamos introduzir noções e fatos sobre o framing de um enlaçamento e sobre o número de enlaçamentos. Para uma introdução à teoria de nós veja [6].

Definição 4.7.1. Considere um enlaçamento orientado $L=K_{1} \cup \cdots \cup K_{m}$ em $S^{3}$. Definimos uma vizinhança tubular da componente $K_{i}$ como sendo um mergulho $T_{i}$ : $D^{2} \times S^{1} \rightarrow S^{3}$ tal que

$$
T_{i}\left(\{0\} \times S^{1}\right)=K_{i}
$$

Onde $D^{2}$ denota o disco unitário no plano dos números complexos $\mathbb{C}$.

Um framing de L é uma coleção $\left\{T_{i}: D^{2} \times S^{1} \rightarrow S^{3}\right\}$ de mergulhos tais que $T_{i}$ é uma vizinhança tubular de $K_{i}$, para $i=1, \ldots, m, e$

$$
T_{i}\left(D^{2} \times S^{1}\right) \cap T_{j}\left(D^{2} \times S^{1}\right) \neq \emptyset \text {, se } i \neq j
$$

$A$ longitude da componente $K_{i}$ é uma curva orientada, fechada e simples $\lambda_{i}$ em $S^{3}$ tal que

$$
\lambda_{i}=T_{i}\left(\{1\} \times S^{1}\right)
$$


Dado um nó $K$, identificamos $H_{1}(K):=H_{1}\left(S^{3} \backslash K\right)$ com $\mathbb{Z}$, de maneira que $1 \in \mathbb{Z}$ é representado pelo 1-ciclo da figura 4.19.

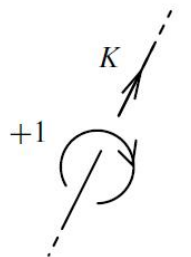

Figura 4.19: 1-ciclo.

Definição 4.7.2. Sejam $K_{1}$ e $K_{2}$ dois nós orientados disjuntos em $S^{3}$. Definimos o número de enlaçamentos $l k\left(K_{1}, K_{2}\right) \in \mathbb{Z}$ como a classe $\left[K_{1}\right] \in H_{1}\left(K_{2}\right)=\mathbb{Z}$.

O número de enlaçamentos $l k\left(K_{1}, K_{2}\right)$ pode ser calculado a partir de qualquer projeção regular do enlaçamento $K_{1} \cup K_{2}$ contando o sinal dos cruzamentos onde $K_{1}$ passa sobre $K_{2}$, como se indica na figura 4.20 .
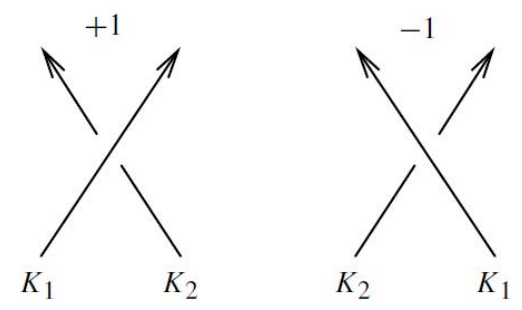

Figura 4.20: Sinal dos cruzamentos.

Dado um enlaçamento orientado $L=K_{1} \cup \cdots \cup K_{m}$ em $S^{3}$, a menos de isotopia, existe um único framing no qual a longitude $\lambda_{i}$ de cada componente $K_{i}$, satisfaz a seguinte condição

$$
\sum_{j=1}^{m} l k\left(\lambda_{i}, K_{i}\right)=0
$$

Vamos nos referir a este framing como framing preferido de $L$. 


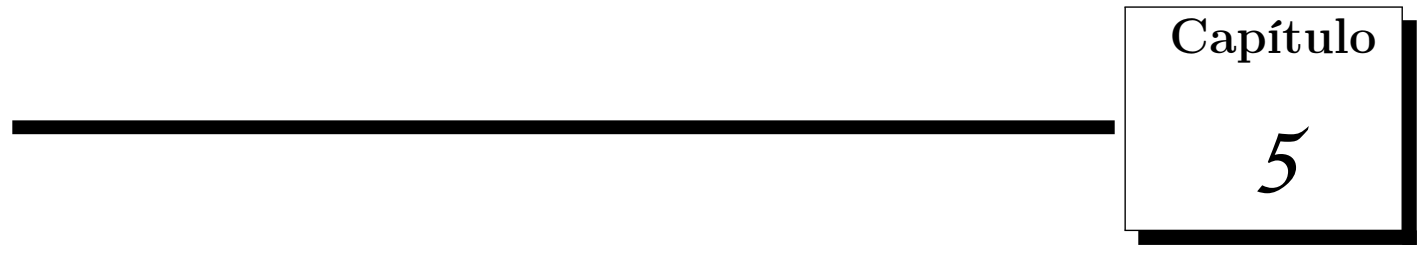

\section{A Representação do Tipo Artin do}

\section{Grupo das Tranças}

Neste capítulo vamos estudar uma nova representação do grupo de tranças que generaliza a representação de Artin dada em 1925 e a representação de Wada dada em 1992 (veja [8]). A partir desta representação construiremos um invariante de enlaçamentos e estudaremos algumas propriedades do mesmo. Também estudaremos uma construção topológica desta nova representação e também do novo invariante.

Neste capítulo, por simplicidade de notação, vamos usar a orientação de uma trança sendo de abaixo para acima, tal como se mostra na figura 5.1.
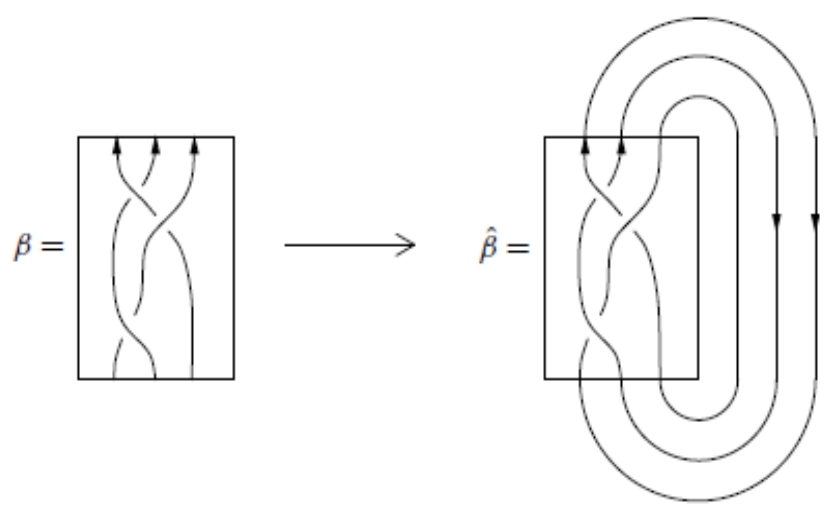

Figura 5.1: Orientação de uma trança $\beta$ e seu fecho. 


\subsection{Definição e Propriedades Elementares}

Considere $B_{n}$ o grupo das tranças de $n$ cordas gerado pelas tranças elementares $\sigma_{1}, . ., \sigma_{n-1}$, as quais satisfazem as relações

$$
\begin{aligned}
\sigma_{k} \sigma_{k+1} \sigma_{k} & =\sigma_{k+1} \sigma_{k} \sigma_{k+1} & & k=1, \ldots, n-2 \\
\sigma_{i} \sigma_{j} & =\sigma_{j} \sigma_{i} & & |i-j|>1
\end{aligned}
$$

Fixe um grupo $H$ e um elemento $h$ em $H$. Considere $n$ cópias $H_{1}, \ldots, H_{n}$ de $H$ e isomorfismos $\phi_{i}: H \rightarrow H_{i}$ e escreva $h_{i}=\phi_{i}(h)$, para $i=1, \ldots, n$. Denote por $H^{* n} \mathrm{o}$ produto livre da família $\left\{H_{i}\right\}_{i=1}^{n}$. Para $k=1, \ldots, n-1$, seja $\tau_{k}: H^{* n} \rightarrow H^{* n}$ definido por

$$
\tau_{k}:\left\{\begin{aligned}
\phi_{k}(y) & \longmapsto h_{k}^{-1} \phi_{k+1}(y) h_{k} \\
\phi_{k+1}(y) & \longmapsto h_{k} \phi_{k}(y) h_{k}^{-1} \\
\phi_{j}(y) & \longmapsto \phi_{j}(y) \quad, \text { se } j \neq k, k+1
\end{aligned}\right.
$$

para $y$ em $H$. Estendendo de forma natural para $H^{* n}$, já que todo elemento de $H^{* n}$ se escreve como um produto de elementos da coleção $\left\{\phi_{i}(y): y \in H, i=1, \ldots n\right\}$, obtemos que $\tau_{k}$ é um homomorfismo. Além disso, $\tau_{k}$ é um automorfismo, pois o homomorfismo (argumento análogo) inverso é definido por

$$
\tau_{k}^{-1}:\left\{\begin{aligned}
\phi_{k}(y) & \longmapsto h_{k+1}^{-1} \phi_{k+1}(y) h_{k+1} \\
\phi_{k+1}(y) & \longmapsto h_{k+1} \phi_{k}(y) h_{k+1}^{-1} \\
\phi_{j}(y) & \longmapsto \phi_{j}(y) \quad, \text { se } j \neq k, k+1
\end{aligned}\right.
$$

Proposição 5.1.1. A aplicação $\sigma_{k} \longmapsto \tau_{k}$, para $k=1, \ldots, n-1$ determina uma represen$\operatorname{tação~} \rho: B_{n} \rightarrow \operatorname{Aut}\left(H^{* n}\right)$.

Demonstração. Basta verificar que $\rho$ respeita as relações de trança, isto é,

$$
\begin{array}{cll}
\tau_{k} \circ \tau_{k+1} \circ \tau_{k}=\tau_{k+1} \circ \tau_{k} \circ \tau_{k+1} & \text { para } & k=1, \ldots, n-2 \\
\tau_{i} \circ \tau_{j}=\tau_{j} \circ \tau_{i} & \text { para } & |i-j|>1, i, j=1, \ldots, n-1
\end{array}
$$

Por simplicidade de notação, escreveremos $y_{i}=\phi_{i}(y)$, para todo $y$ em $H$ e $i=1, \ldots, n$. Seja $k=1, \ldots, n-2$ fixo. Claramente, $\tau_{k} \circ \tau_{k+1} \circ \tau_{k}\left(y_{j}\right)=\tau_{k+1} \circ \tau_{k} \circ \tau_{k+1}\left(y_{j}\right)$, para $j \neq k, k+1, k+2$. Temos 


$$
\begin{aligned}
\tau_{k} \circ \tau_{k+1} \circ \tau_{k}\left(y_{k}\right)= & \tau_{k} \circ \tau_{k+1}\left(h_{k}^{-1} y_{k+1} h_{k}\right) \\
= & \tau_{k}\left(h_{k}^{-1} h_{k+1}^{-1} y_{k+2} h_{k+1} h_{k}\right) \\
= & h_{k}^{-1} h_{k+1}^{-1} h_{k} h_{k}^{-1} y_{k+2} h_{k} h_{k}^{-1} h_{k+1} h_{k} \\
= & h_{k}^{-1} h_{k+1}^{-1} y_{k+2} h_{k+1} h_{k} \\
\tau_{k} \circ \tau_{k+1} \circ \tau_{k}\left(y_{k+1}\right) & =\tau_{k} \circ \tau_{k+1}\left(h_{k} y_{k} h_{k}^{-1}\right) \\
& =\tau_{k}\left(h_{k} y_{k} h_{k}^{-1}\right) \\
& =h_{k}^{-1} h_{k+1} h_{k} h_{k}^{-1} y_{k+1} h_{k} h_{k}^{-1} h_{k+1}^{-1} h_{k} \\
& =h_{k}^{-1} h_{k+1} y_{k+1} h_{k+1}^{-1} h_{k} \\
& =\tau_{k} \circ \tau_{k+1}\left(y_{k+2}\right) \\
\tau_{k} \circ \tau_{k+1} \circ \tau_{k}\left(y_{k+2}\right) & \tau_{k}\left(h_{k+1} y_{k+1} h_{k+1}^{-1}\right) \\
= & h_{k} h_{k} y_{k} h_{k}^{-1} h_{k}^{-1} \\
= & h_{k}^{2} y_{k} h_{k}^{-2}
\end{aligned}
$$

e por outro lado

$$
\begin{aligned}
\tau_{k+1} \circ \tau_{k} \circ \tau_{k+1}\left(y_{k}\right)= & \tau_{k+1} \circ \tau_{k}\left(y_{k}\right) \\
= & \tau_{k+1}\left(h_{k}^{-1} y_{k+1} h_{k}\right) \\
= & h_{k}^{-1} h_{k+1}^{-1} y_{k+2} h_{k+1} h_{k} \\
\tau_{k+1} \circ \tau_{k} \circ \tau_{k+1}\left(y_{k+1}\right) & =\tau_{k+1} \circ \tau_{k}\left(h_{k+1}^{-1} y_{k+2} h_{k+1}\right) \\
& =\tau_{k+1}\left(h_{k}^{-1} y_{k+2} h_{k}\right) \\
& =h_{k}^{-1} h_{k+1} y_{k+1} h_{k+1}^{-1} h_{k} \\
& =\tau_{k+1} \circ \tau_{k}\left(h_{k+1} y_{k+1} h_{k+1}^{-1}\right) \\
& =\tau_{k+1}\left(h_{k} h_{k} y_{k} h_{k}^{-1} h_{k}^{-1}\right) \\
\tau_{k+1} \circ \tau_{k} \circ \tau_{k+1}\left(y_{k+2}\right) & h_{k}^{2} y_{k} h_{k}^{-2}
\end{aligned}
$$

Portanto, demonstramos que $\tau_{k} \circ \tau_{k+1} \circ \tau_{k}=\tau_{k+1} \circ \tau_{k} \circ \tau_{k+1}$ para $k=1, \ldots, n-2$.

Agora vamos mostrar que $\tau_{i} \circ \tau_{j}=\tau_{j} \circ \tau_{i}$ para $|i-j|>1$, onde $i, j=1, \ldots, n-1$. Sem perda de generalidade suponha $i>j+1$. Então, $\tau_{i}\left(y_{j}\right)=y_{j}, \tau_{i}\left(y_{j+1}\right)=y_{j+1}, \tau_{j}\left(y_{i}\right)=y_{i}$ e $\tau_{j}\left(y_{i+1}\right)=y_{i+1}$. Portanto é claro que

$$
\tau_{i} \circ \tau_{j}\left(y_{j}\right)=\tau_{j} \circ \tau_{i}\left(y_{j}\right) \text { e } \tau_{i} \circ \tau_{j}\left(y_{j+1}\right)=\tau_{j} \circ \tau_{i}\left(y_{j+1}\right)
$$


Por outro lado, para $t \neq j, j+1$, temos

$$
\tau_{i} \circ \tau_{j}\left(y_{t}\right)=\tau_{i}\left(y_{t}\right)=\tau_{j} \circ \tau_{i}\left(y_{t}\right)
$$

Assim, $\tau_{i} \circ \tau_{j}=\tau_{j} \circ \tau_{i}$.

Definição 5.1.2. A representação da proposição anterior é chamada representação de tipo Artin associada ao par $(H, h)$.

Exemplo 5.1.3. Seja $H$ um grupo arbitrário e suponha $h=e_{H}$. Então

$$
\tau_{k}: \begin{cases}\phi_{k}(y) & \longmapsto \phi_{k+1}(y) \\ \phi_{k+1}(y) & \longmapsto \phi_{k}(y) \\ \phi_{j}(y) & \longmapsto \phi_{j}(y) \quad \text {, se } j \neq k, k+1\end{cases}
$$

Defina $\Sigma: \operatorname{Aut}\left(H^{* n}\right) \rightarrow S_{n}$ por $\Sigma\left(\tau_{k}\right)=(k k+1)$. Como $S_{n}=\langle(i i+1): i=1, \ldots n-1\rangle$ então $\Sigma \circ \rho$ é a representação por permutações do grupo de tranças cuja imagen é $S_{n} e$ $\operatorname{ker}(\Sigma \circ \rho)=P_{n}$ é o subgrupo de $B_{n}$ das tranças puras (Ver [3], página 19).

Exemplo 5.1.4. Seja $H=(\mathbb{Z},+)$ e $h=1$. Então $H^{* n}=\mathbb{F}_{n}$, o grupo livre de posto $n, e$

$$
\tau_{k}:\left\{\begin{array}{lll}
x_{k} & \longmapsto x_{k}^{-1} x_{k+1} x_{k} & \\
x_{k+1} & \longmapsto x_{k} & \\
x_{j} & \longmapsto & x_{j}
\end{array} \text {, se } j \neq k, k+1\right.
$$

Neste caso $\rho$ é a clássica representação de Artin introduzida pelo mesmo em 1925.

Exemplo 5.1.5. Outro caso interessante é quando $H=\mathbb{Z}$ e h é um inteiro diferente de 1 e 0. Este caso foi estudado por Wada em 1992 na sua construção de invariantes de enlaçamentos (ver [8]).

Agora, passamos a estudar as diferentes propriedades da representação de tipo Artin.

Definição 5.1.6. Seja $\rho: B_{n} \rightarrow$ Aut $\left(H^{* n}\right)$ a representação de tipo Artin associada ao par $(H, h)$. Seja $\beta$ uma n-trança. Vamos denotar por $\Gamma(\beta)=\Gamma_{(H, h)}(\beta)$ o quociente de 
$H^{* n}$ pelas relações

$$
g=\rho(\beta)(g), g \in H^{* n}
$$

Isto é,

$$
\Gamma(\beta)=H^{* n} /\left\langle g(\rho(\beta)(g))^{-1}: g \in H^{* n}\right\rangle^{H^{* n}}
$$

Para uma $n$-trança $\beta$, denotamos por $\widehat{\beta}$ o enlaçamento orientado (mais precisamente, a classe de equivalencia de elaçamentos orientados) representado pelo fecho da trança, como foi definido anteriormente (Ver [2]).

\subsection{Enlaçamentos e Invariantes de Enlaçamentos}

Seja $H$ um grupo, $h \in H$ e $\rho: B_{n} \rightarrow A u t\left(H^{* n}\right)$ a representação de tipo Artin associada ao $\operatorname{par}(H, h)$. Lembremos que $H^{* n}=H_{1} * \ldots * H_{n}$ com $H_{1}, \ldots, H_{n}$ cópias de $H$ e $\phi_{i}: H \rightarrow H_{i}$ isomorfismos dados para $i=1, \ldots, n$.

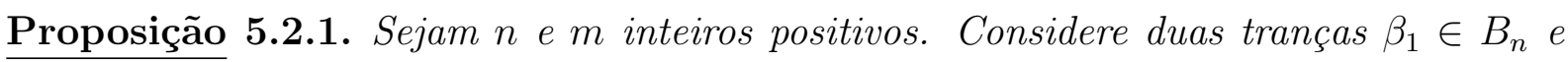
$\beta_{2} \in B_{m}$. Se $\widehat{\beta}_{1}=\widehat{\beta}_{2}$ então $\Gamma_{(H, h)}\left(\beta_{1}\right) \simeq \Gamma_{(H, h)}\left(\beta_{2}\right)$.

Demonstração. Queremos provar que $\Gamma_{(H, h)}$ é um invariante de enlaçamentos. Pelo teorema de Markov (teorema 4.5.2), basta provar que para uma $n$-trança qualquer $\beta \in B_{n}$, temos

(1) $\Gamma\left(\alpha^{-1} \beta \alpha\right) \simeq \Gamma(\beta)$, para toda $n$-trança $\alpha$ em $B_{n}$.

(2) $\Gamma\left(\beta \sigma_{n}\right) \simeq \Gamma(\beta)$

(3) $\Gamma\left(\beta \sigma_{n}^{-1}\right) \simeq \Gamma(\beta)$

onde $\beta \sigma_{n}$ e $\beta \sigma_{n}^{-1}$ são vistas como tranças em $B_{n+1}$.

Observe que se $\beta$ está em $B_{n}$ e $n \leq m$, então a ação de $\beta$ sob $\rho$ sobre $H^{* m}$ é trivial nos fatores livres $H_{n+1}, \ldots, H_{m}$, pois a ação de $\sigma_{k}$ é trivial sobre os fatores livres $H_{n+1}, \ldots, H_{m}$, para $k=1, \ldots, n-2$. Por simplicidade de notação, escrevemos $\beta(g)$ em vez de $\rho(\beta)(g)$ e $\sigma_{k}$ em vez de $\tau_{k}$.

Para provar (1), note que, para $\beta \in B_{n}$, o grupo $\Gamma(\beta)$ é definido como o quociente de $H^{* n}$ pelas relações $g=\beta(g)$ para todo $g \in H^{* n}$. Como, para $\alpha \in B_{n}$, a relação 
$g=\alpha^{-1} \beta \alpha(g)$ é equivalente à relação $\alpha(g)=\beta(\alpha(g))$, e $\alpha$ é um automorfismo de $H^{* n}$, segue que $\Gamma\left(\alpha^{-1} \beta \alpha\right)$ é definido pelo mesmo conjunto de relações que $\Gamma(\beta)$. Portanto, os grupos $\Gamma\left(\alpha^{-1} \beta \alpha\right)$ e $\Gamma(\beta)$ são isomorfos.

Agora provaremos (2). O grupo $\Gamma\left(\beta \sigma_{n}\right)$ pode ser visto como o quociente de $H^{*(n+1)}$ pelas relações

$$
R(i, x): \phi_{i}(x)=\beta \sigma_{n}\left(\phi_{i}(x)\right) \operatorname{com} i=1, \ldots, n+1, x \in H
$$

Observe que

(i) $\sigma_{n}\left(\phi_{n}(x)\right)=h_{n}^{-1} \phi_{n+1}(x) h_{n}$

(ii) $\sigma_{n}\left(\phi_{n+1}(x)\right)=h_{n} \phi_{n}(x) h_{n}^{-1}$

Portanto, $R(n+1, x)$ é equivalente a

$$
R^{\prime}(n+1, x): \phi_{n+1}(x)=\beta\left(h_{n}\right) \beta\left(\phi_{n}(x)\right) \beta\left(h_{n}^{-1}\right)
$$

onde $\beta\left(h_{n}\right) \beta\left(\phi_{n}(x)\right) \beta\left(h_{n}^{-1}\right)=\beta\left(h_{n} \phi_{n}(x) h_{n}^{-1}\right)$ está em $H^{* n}$. Logo, $\Gamma\left(\beta \sigma_{n}\right)$ é gerado pela imagen de $H^{* n}$. Além disso,

$$
\begin{aligned}
\phi_{n}(x) & =\beta \sigma_{n}\left(\phi_{n}(x)\right) \\
& =\beta\left(h_{n}^{-1} \phi_{n+1}(x) h_{n}\right) \\
& =\beta\left(h_{n}^{-1}\right) \phi_{n+1}(x) \beta\left(h_{n}\right) \\
& =\beta\left(h_{n}^{-1}\right) \beta\left(h_{n}\right) \beta\left(\phi_{n}(x)\right) \beta\left(h_{n}^{-1}\right) \beta\left(h_{n}\right) \\
& =\beta\left(\phi_{n}(x)\right)
\end{aligned}
$$

onde, na terceira igualdade usamos que $\beta \in B_{n}$ e na quarta igualdade usamos $R^{\prime}(n+1, x)$.

Por outro lado, a relação $R(i, x)$ é equivalente a $R^{\prime}(i, x): \phi_{i}(x)=\beta\left(\phi_{i}(x)\right)$, para $i=1, \ldots, n-1$, pois $\sigma_{n}$ é a identidade sobre os fatores livres $H_{1}, \ldots, H_{n-1}$. Portanto, $\Gamma\left(\beta \sigma_{n}\right) \simeq \Gamma(\beta)$.

Finalmente provaremos (3). Primeiro note que para todo $g$ em $H^{* n}$ temos

$$
g=\beta(g) \Leftrightarrow g=\beta^{-1}(g)
$$


pois $\beta$ é um automorfismo de $H^{* n}$. Logo, $\Gamma(\beta) \simeq \Gamma\left(\beta^{-1}\right)$. Portanto,

$$
\begin{aligned}
\Gamma\left(\beta \sigma_{n}^{-1}\right) & \simeq \Gamma\left(\sigma_{n} \beta^{-1}\right) \\
& \left.\simeq \Gamma\left(\beta^{-1} \sigma_{n}\right), \text { por } 1\right) \\
& \left.\simeq \Gamma\left(\beta^{-1}\right), \text { por } 2\right) \\
& \simeq \Gamma(\beta)
\end{aligned}
$$

Definição 5.2.2. Seja L um enlaçamento orientado. Definimos

$$
\Gamma_{(H, h)}(L):=\Gamma_{(H, h)}(\beta)
$$

onde $\beta$ é uma trança tal que $L=\widehat{\beta}$.

Note que a proposição 5.2.1 mostra que $\Gamma_{(H, h)}(L)$ não depende (da classe de equivalência) da trança $\beta$, ou seja, $\Gamma_{(H, h)}(L)$ está bem definido.

\subsection{Construção Topológica do Invariante de Enlaçamen-}

\section{tos}

Seja $X$ um complexo CW. Considere $P_{0} \in X$ como ponto base e seja $\alpha:[0,1] \rightarrow X$ um laço baseado em $P_{0}$. Nesta seção vamos dar uma realização topológica da representação de tipo Artin de $B_{n}$ associada ao par $(H, h)=\left(\pi_{1}\left(X, p_{0}\right),[\alpha]\right)$, e a seguir deduziremos uma construção topológica do invariante de enlaçamentos $\Gamma_{(H, h)}(\cdot)$ da seção anterior.

Denote por $\mathbf{D}=D\left(\frac{n+1}{2}, \frac{n+1}{2}\right)$ o disco no conjunto dos números complexos, $\mathbb{C}$, centrado em $\frac{n+1}{2}$ e de raio $\frac{n+1}{2}$. Vamos construir o espaço $Y$ obtido de $\mathbf{D}$ fazendo $n$ buracos em D e colando uma cópia de $X$ em cada buraco, mediante uma identificação da fronteira circular do buraco com o laço $\alpha$ em $X$. Mais precisamente: considere um número $\varepsilon>0$ suficientemente pequeno (requeremos $\varepsilon<\frac{1}{8}$ ), e seja

$$
Y^{\prime}=\mathbf{D} \backslash\left(\bigcup_{k=1}^{n} \stackrel{\circ}{D}(k, \varepsilon)\right)
$$

onde $\stackrel{\circ}{D}(k, \varepsilon)$ denota o interior do disco centrado em $k$ e de raio $\varepsilon$. Tome $n$-cópias $X_{1}, \ldots, X_{n}$ 
de $X$. Para $k=1, \ldots, n$, considere $f_{k}: X \rightarrow X_{k}$ homeomorfismo e escreva $\alpha_{k}=f_{k} \circ \alpha$. Então

$$
Y=\left(Y^{\prime} \dot{\bigcup}\left(\bigcup_{k=1}^{n} X_{k}\right)\right) / \sim
$$

onde é a identificação definida por

$$
\alpha_{k}(t) \sim k+\varepsilon e^{2 \pi i t}, k=1, \ldots, n, t \in I
$$

Finalmente, escolha um ponto base $Q_{0} \in \partial \mathbf{D}$ para $Y$. O seguinte resultado é uma consequência direta da construção anterior.

Lema 5.3.1. Seja $H=\pi_{1}\left(X, P_{0}\right)$ e sejam $H_{1}, \ldots, H_{n}$ cópias de $H$. Então

$$
\pi_{1}\left(Y, Q_{0}\right) \simeq H_{1} * \ldots * H_{n}
$$

Demonstração. Note que $\pi_{1}\left(Y, Q_{0}\right)$ não depende de $Q_{0}$ em $\partial \mathbf{D}$ pois o caminho $\gamma:[0,1] \rightarrow$ $Y$, definido por $\gamma(t)=\frac{n+1}{2}+\frac{n+1}{2} e^{2 \pi i t}$ une todos os pontos de $\partial \mathbf{D}$. Agora faremos a demonstração por indução sobre $n$.

Suponha $n=2$. Escreva $Y=A \cup B$ onde

$$
\left.A=\left\{z: \Re(z)<\frac{3}{2}+\delta\right\} \cap\left(Y^{\prime} \cup X_{1}\right) \text { e } B=\left\{z: \Re(z)>\frac{3}{2}-\delta\right\} \cap\left(Y^{\prime} \cup X_{2}\right)\right\}
$$

para algum $\delta>0$ apropiado. Observe que $A \cap B$ é simplesmente conexo, logo, pelo teorema de Seifert-Van Kampen temos

$$
\pi_{1}(Y) \simeq \pi_{1}(A) * \pi_{1}(B)
$$

Defina $r_{1}: A \rightarrow X_{1}$ por

$$
r_{1}:\left\{\begin{array}{rll}
x & \longmapsto x, & x \in X_{1} \\
d & \longmapsto \frac{\epsilon}{|1-d|}(1-d)+1, \quad d \in A \backslash X_{1}
\end{array}\right.
$$

Observe que $r_{1}$ é contínua (lema da colagem) e que $\left.r_{1}\right|_{X_{1}}=I d_{X_{1}}$. Portanto, $X_{1}$ é um 
retrato de $A$. Além disso, a aplicação $F: A \times[0,1] \rightarrow A$ definida por

$$
\begin{array}{lrl}
F(d, t) & =(1-t) d+\operatorname{tr}_{1}(d) & , d \in A \backslash X_{1}, t \in[0,1] \\
F(x, t) & =x & , x \in X_{1}, t \in[0,1]
\end{array}
$$

é uma homotopia entre $i \circ r_{1}$ e $I d_{A}$ relativa a $X_{1}$, onde $i: X_{1} \hookrightarrow A$ é a inclusão. Portanto, $X_{1}$ é um retrato por deformação de $A$. Então, pela proposição 3.5.8 temos

$$
\pi_{1}\left(X, P_{0}\right) \simeq \pi_{1}\left(A, Q_{0}\right)
$$

Analogamente temos

$$
\pi_{1}\left(X, P_{0}\right) \simeq \pi_{1}\left(B, Q_{0}\right)
$$

Portanto, $\pi_{1}\left(Y, Q_{0}\right) \simeq \pi_{1}\left(X, P_{0}\right) * \pi_{1}\left(X, P_{0}\right)$.

O caso geral segue por indução sobre $n$.

Vamos mostrar que o grupo de $n$-tranças $B_{n}$ atua sobre $Y$ a menos de isotopia relativa à fronteira de $\mathbf{D}$ de modo que a ação induzida sobre $\pi_{1}(Y)$ é a representação de tipo Artin associada ao par $(H, h)=\left(\pi_{1}\left(X, P_{0}\right),[\alpha]\right)$.

Definição 5.3.2. Seja $\xi e m \mathbb{C} e 0<r<R$. Uma meia torção de Dehn $T=T(\xi, r, R)$, é uma aplicação do plano complexo $\mathbb{C}$, definida por

$$
T\left(\xi+\rho e^{i \theta}\right)= \begin{cases}\xi+\rho e^{i(\theta-\pi)} & 0 \leq \rho \leq r \\ \xi+\rho e^{i(\theta-t \pi)} & r \leq \rho \leq R e t=\frac{R-\rho}{R-r} \\ \xi+\rho e^{i \theta} & \rho \geq R\end{cases}
$$
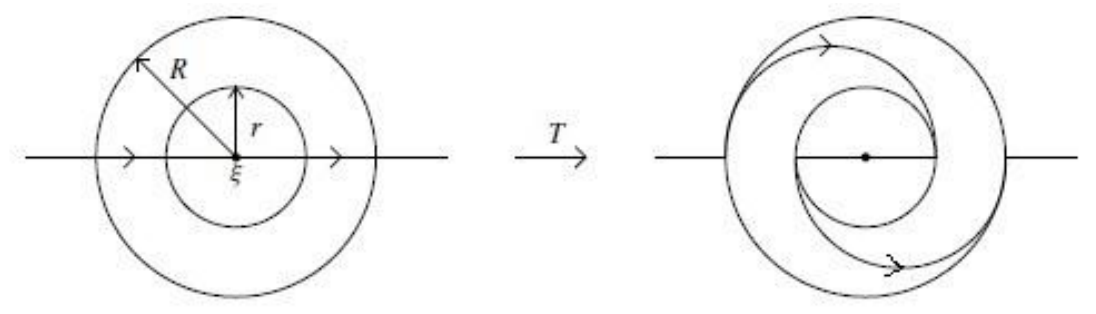

Figura 5.2: Meia torção de Dehn. 
Observação 5.3.3. A meia torção de Dehn é um homeomorfismo e sua inversa $T^{-1}$ é dada por

$$
T^{-1}\left(\xi+\rho e^{i \theta}\right)= \begin{cases}\xi+\rho e^{i(\theta+\pi)} & , \text { se } 0 \leq \rho \leq r \\ \xi+\rho e^{i(\theta+t \pi)} & , \text { se } r \leq \rho \leq R \text { e } t=\frac{R-\rho}{R-r} \\ \xi+\rho e^{i \theta} & , \text { se } \rho \geq R\end{cases}
$$

Para cada $k=1, \ldots, n$, seja $T_{k}^{\mathbf{D}}: \mathbf{D} \rightarrow \mathbf{D}$ o homeomorfismo definido por

$$
T_{k}^{\mathbf{D}}=T(k, \varepsilon, 2 \varepsilon)^{-3} \circ T(k+1, \varepsilon, 2 \varepsilon)^{-1} \circ T\left(k+\frac{1}{2}, \frac{1}{2}+\varepsilon, \frac{1}{2}+2 \varepsilon\right)
$$

Intuitivamente, $T_{k}^{\mathbf{D}}$ transforma o interior de $D\left(k+\frac{1}{2}, \frac{1}{2}+2 \epsilon\right)$ como segue: imagine que uma mão segura a borda do disco $D\left(k+\frac{1}{2}, \frac{1}{2}+2 \epsilon\right)$ e a outra segura a borda do disco $D\left(k+\frac{1}{2}, \frac{1}{2}+\epsilon\right)$, logo, gira-se em sentido horário o disco $D\left(k+\frac{1}{2}, \frac{1}{2}+\epsilon\right)$ em $\pi$ radianos, mexendo os pontos do interior de $D\left(k+\frac{1}{2}, \frac{1}{2}+2 \epsilon\right)$ segundo a definição da meia torção de Dhen (ver figura 5.2). Analogamente, segura-se a borda do disco $D(k+1,2 \epsilon)$ e gira-se o disco $D(k+1, \epsilon)$ em sentido horário em $\pi$ radianos mexendo-se agora somente os pontos do interior de $D(k+1,2 \epsilon)$. Finalmente, segura-se a borda do disco $D(k, 2 \epsilon)$ e dá-se três giros de $\pi$ radianos ao disco $D(k, \epsilon)$ em sentido antihorário. Esta é uma forma intuitiva de explicar como a aplicação $T_{k}^{\mathbf{D}}$ atua em D. Na figura 5.3, cada seta simboliza o giro descrito uma vez.

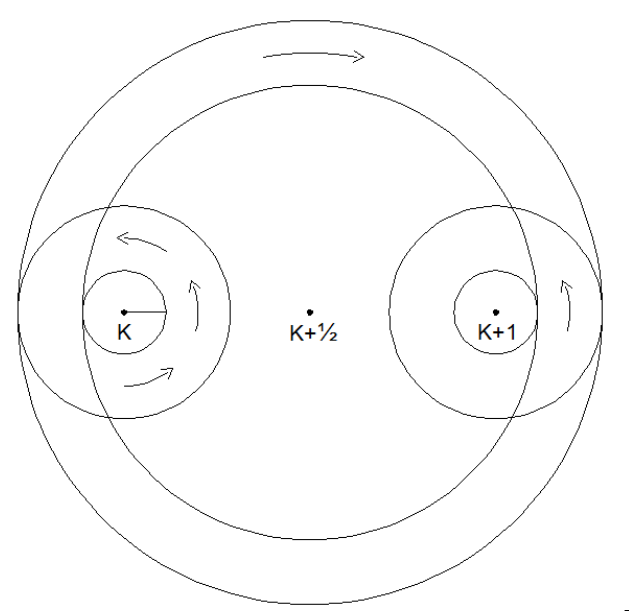

Figura 5.3: Descrição da aplicação $T_{k}^{\mathbf{D}}$. 
Observe que $\bigcup_{j=1}^{n} \mathbf{D}(j, \varepsilon)$ fica invariante sob $T_{k}^{\mathbf{D}} ;$ isto é,

$$
T_{k}^{\mathbf{D}}\left(\bigcup_{j=1}^{n} \mathbf{D}(j, \varepsilon)\right)=\bigcup_{j=1}^{n} \mathbf{D}(j, \varepsilon)
$$

Portanto, sua restrição $T_{k}^{\prime}: Y^{\prime} \rightarrow Y^{\prime}$ também é um homeomorfismo. Temos então a seguinte proposição.

Proposição 5.3.4. Com as notações anteriores temos:

(1) $T_{k}^{\prime} T_{k+1}^{\prime} T_{k}^{\prime}$ é isotópica a $T_{k+1}^{\prime} T_{k}^{\prime} T_{k+1}^{\prime}$ relativamente a $\partial \mathbf{D}$, para $k=1, \ldots, n-2$.

(2) $T_{k}^{\prime} T_{l}^{\prime}$ é isotópica a $T_{l}^{\prime} T_{k}^{\prime}$ relativamente a $\partial \mathbf{D}$, para $|k-l|>1$.

Demonstração. Defina para cada $t \in[0,1]$ uma aplicação $T_{t}=T_{t}(\xi, r, R): \mathbb{C} \rightarrow \mathbb{C}$ por

$$
T_{t}\left(\xi+\rho e^{i \theta}\right)= \begin{cases}\xi+\rho e^{i(\theta-t \pi)} & , \text { se } 0 \leq \rho \leq r \\ \xi+\rho e^{i\left(\theta-t \frac{R-\rho}{R-r} \pi\right)} & , \text { se } r \leq \rho \leq R \\ \xi+\rho e^{i \theta} & , \text { se } \rho \geq R\end{cases}
$$

$T_{t}$ é um homeomorfismo pois é contínua pelo lema da colagem e sua inversa é definida por

$$
T_{t}^{-1}\left(\xi+\rho e^{i \theta}\right)= \begin{cases}\xi+\rho e^{i(\theta+t \pi)} & , \text { se } 0 \leq \rho \leq r \\ \xi+\rho e^{i\left(\theta+t \frac{R-\rho}{R-r} \pi\right)} & , \text { se } r \leq \rho \leq R \\ \xi+\rho e^{i \theta} & , \text { se } \rho \geq R\end{cases}
$$

que também é contínua pelo lema da colagem.

Observe que $T_{0}=I d_{\mathbb{C}}$ e $T_{1}=T(\xi, r, R)$.

Agora vamos demonstrar (1) e (2). Para demonstrar (1) defina a aplicação

$$
h: Y^{\prime} \times[0,1] \rightarrow Y^{\prime}
$$


por

$$
\begin{aligned}
h(\cdot, t)= & T_{1-t}(k, \epsilon, 2 \epsilon)^{-3} \circ T_{1-t}(k+1, \epsilon, 2 \epsilon)^{-1} \circ T_{1-t}\left(k+\frac{1}{2}, \frac{1}{2}+\epsilon, \frac{1}{2}+2 \epsilon\right) \circ \\
& \circ T_{1-t}(k+1, \epsilon, 2 \epsilon)^{-3} \circ T_{1-t}(k+2, \epsilon, 2 \epsilon)^{-1} \circ T_{1-t}\left(k+\frac{3}{2}, \frac{1}{2}+\epsilon, \frac{1}{2}+2 \epsilon\right) \circ \\
& \circ T_{1-t}(k, \epsilon, 2 \epsilon)^{-3} \circ T_{1-t}(k+1, \epsilon, 2 \epsilon)^{-1} \circ T_{1-t}\left(k+\frac{1}{2}, \frac{1}{2}+\epsilon, \frac{1}{2}+2 \epsilon\right) \circ \\
& \circ T_{t}(k+1, \epsilon, 2 \epsilon)^{-3} \circ T_{t}(k+2, \epsilon, 2 \epsilon)^{-1} \circ T_{t}\left(k+\frac{3}{2}, \frac{1}{2}+\epsilon, \frac{1}{2}+2 \epsilon\right) \circ \\
& \circ T_{t}(k, \epsilon, 2 \epsilon)^{-3} \circ T_{t}(k+1, \epsilon, 2 \epsilon)^{-1} \circ T_{t}\left(k+\frac{3}{2}, \frac{1}{2}+\epsilon, \frac{1}{2}+2 \epsilon\right) \circ \\
& \circ T_{t}(k+1, \epsilon, 2 \epsilon)^{-3} \circ T_{t}(k+2, \epsilon, 2 \epsilon)^{-1} \circ T_{t}\left(k+\frac{3}{2}, \frac{1}{2}+\epsilon, \frac{1}{2}+2 \epsilon\right)(\cdot)
\end{aligned}
$$

É claro que para todo $t$ em $[0,1], h(\cdot, t)$ é um homeomorfismo que fixa a fronteira do disco D ponto a ponto, e além disso, $h(\cdot, 0)=\left(T_{k}^{\prime} \circ T_{k+1}^{\prime} \circ T_{l}^{\prime}\right)(\cdot)$ e $h(\cdot, 1)=\left(T_{k+1}^{\prime} \circ T_{k}^{\prime} \circ T_{k+1}^{\prime}\right)(\cdot)$.

Defina

$$
H: Y^{\prime} \times[0,1] \rightarrow Y^{\prime} \times[0,1] \text { por } H(\cdot, t)=(h(\cdot, t), t)
$$

É claro que $H$ é a isotopia procurada.

Analogamente, para demonstrar (2), considere $|k-l|>1$ e defina

$$
g: Y^{\prime} \times[0,1] \rightarrow Y^{\prime}
$$

por

$$
\begin{aligned}
g(\cdot, t)= & T_{1-t}(k, \epsilon, 2 \epsilon)^{-3} \circ T_{1-t}(k+1, \epsilon, 2 \epsilon)^{-1} \circ T_{1-t}\left(k+\frac{1}{2}, \frac{1}{2}+\epsilon, \frac{1}{2}+2 \epsilon\right) \circ \\
& T_{1-t}(l, \epsilon, 2 \epsilon)^{-3} \circ T_{1-t}(l+1, \epsilon, 2 \epsilon)^{-1} \circ T_{1-t}\left(l+\frac{1}{2}, \frac{1}{2}+\epsilon, \frac{1}{2}+2 \epsilon\right) \circ \\
& T_{t}(l, \epsilon, 2 \epsilon)^{-3} \circ T_{t}(l+1, \epsilon, 2 \epsilon)^{-1} \circ T_{t}\left(l+\frac{1}{2}, \frac{1}{2}+\epsilon, \frac{1}{2}+2 \epsilon\right) \circ \\
& T_{t}(k, \epsilon, 2 \epsilon)^{-3} \circ T_{t}(k+1, \epsilon, 2 \epsilon)^{-1} \circ T_{t}\left(k+\frac{1}{2}, \frac{1}{2}+\epsilon, \frac{1}{2}+2 \epsilon\right)
\end{aligned}
$$

Defina

$$
G: Y^{\prime} \times[0,1] \rightarrow Y^{\prime} \times[0,1] \text { por } G(\cdot, t)=(g(\cdot, t), t)
$$

É fácil ver que $G$ é a isotopia procurada entre $T_{k} \circ T_{l}$ e $T_{l} \circ T_{k}$. 
Usando a família de funções $\left\{T_{t}\right\}_{\{t \in[0,1]\}}$ na demonstração da proposição anterior, obtemos o seguinte corolário.

Corolário 5.3.5. Para cada $k=1, \ldots, n, T_{k}$ é isotópica à $I d_{\mathbf{D}}$ relativamente à $\partial \mathbf{D}$.

Observação 5.3.6. $T_{k}^{\prime}$ fixa $\partial \mathbf{D}$ ponto a ponto e transforma os restantes pontos de $\delta Y^{\prime}$ como segue

$$
T_{k}^{\prime}\left(j+\epsilon e^{i \theta}\right)= \begin{cases}j+\epsilon e^{i \theta} & , \text { se } j \neq k, k+1 \\ k+1+\epsilon e^{i \theta} & , \text { se } j=k \\ k+\epsilon e^{i \theta} & , \text { se } j=k+1\end{cases}
$$

Pela observação 5.3.6, podemos estender $T_{k}^{\prime}$ a um homeomorfismo $T_{k}: Y \rightarrow Y$. Defina $T_{k}: Y \rightarrow Y$ para todo $x$ em $X$ por

$$
T_{k}\left(f_{j}(x)\right)= \begin{cases}f_{j}(x) & , \text { se } j \neq k, k+1 \\ f_{k+1}(x) & , \text { se } j=k \\ f_{k}(x) & , \text { se } j=k+1\end{cases}
$$

e $\left.T_{k}\right|_{Y^{\prime}}=T_{k}^{\prime}$. $T_{k}$ é contínua com inversa contínua (lema da colagem). Logo, $T_{k}$ é um homeomorfismo. Além disso, $T_{k}$ fixa ponto a ponto a fronteira do disco D. Temos assim, a seguinte proposição.

Proposição 5.3.7. Com as notações anteriores:

(1) $T_{k} \circ T_{k+1} \circ T_{k}$ é isotópico a $T_{k+1} \circ T_{k} \circ T_{k+1}$ relativamente à $\partial \mathbf{D}$, para $k=1, \ldots, n-2$.

(2) $T_{k} \circ T_{l}$ é isotópico a $T_{l} \circ T_{k}$ relativamente à $\partial \mathbf{D}$, para $k, l=1, \ldots, n-1$ com $|k-l|>1$.

Demonstração. Observe que para $k=1, \ldots, n-2$ e $x$ em $X$ temos

$$
\left(T_{k} \circ T_{k+1} \circ T_{k}\right)\left(f_{j}(x)\right)=\left(T_{k+1} \circ T_{k} \circ T_{k+1}\right)\left(f_{j}(x)\right)= \begin{cases}f_{j}(x), & \text { se } j \neq k, k+1, k+2 \\ f_{k+2}(x) & , \text { se } j=k \\ f_{k+1}(x), & \text {, se } j=k+1 \\ f_{k}(x), & \text {, se } j=k+2\end{cases}
$$

Considere a aplicação

$$
H: Y \times[0,1] \rightarrow Y \times[0,1]
$$


sendo uma extensão da aplicação dada por (5.3.10) na demonstração da proposição 5.3.4, de maneira que para $t \in[0,1]$ e $x$ em $X$ arbitrário, temos

$$
H\left(f_{j}(x), t\right)=\left(\left(T_{k} \circ T_{k+1} \circ T_{k}\right)\left(f_{j}(x)\right), t\right)
$$

é fácil ver que $H$ é a isotopia requerida entre $T_{k} \circ T_{k+1} \circ T_{k}$ e $T_{k+1} \circ T_{k} \circ T_{k+1}$. Fica demonstrada a parte (1).

Analogamente vamos provar (2). Observe que para $k, l=1, \ldots, n-1$ com $|k-l|>1$ temos

$$
\left(T_{k} \circ T_{l}\right)\left(f_{j}(x)\right)=\left(T_{l} \circ T_{k}\right)\left(f_{j}(x)\right)= \begin{cases}f_{j}(x) & , \text { se } j \neq k, k+1, l, l+1 \\ f_{k+1}(x) & , \text { se } j=k \\ f_{k}(x) & , \text { se } j=k+1 \\ f_{l+1}(x) & , \text { se } j=l \\ f_{l}(x) & , \text { se } j=l+1\end{cases}
$$

Defina a aplicação

$$
G: Y \times[0,1] \rightarrow Y \times[0,1]
$$

para $t \in[0,1]$ como em (5.3.12) sobre $Y^{\prime}$, e para $x$ em $X$ arbitrário por

$$
G\left(f_{j}(x), t\right)=\left(\left(T_{k} \circ T_{l}\right)\left(f_{j}(x)\right), t\right)
$$

É claro que $G$ é uma isotopia entre $T_{k} \circ T_{l}$ e $T_{l} \circ T_{k}$. Segue o resultado.

Temos então, pelas propriedades do homomorfismo induzido por uma função contínua, que $\left(T_{k}\right)_{*}$ é um automorfismo de $\pi_{1}\left(Y, Q_{0}\right)$, para todo $k=1, \ldots, n-1$, e pela proposição anterior, este automorfismo satisfaz as relações

(1) $\left(T_{k}\right)_{*} \circ\left(T_{k+1}\right)_{*} \circ\left(T_{k}\right)_{*}=\left(T_{k+1}\right)_{*} \circ\left(T_{k}\right)_{*} \circ\left(T_{k+1}\right)_{*}$, para $k=1, \ldots, n-2$.

(2) $\left(T_{k}\right)_{*} \circ\left(T_{l}\right)_{*}=\left(T_{l}\right)_{*} \circ\left(T_{k}\right)_{*}$, para $k, l=1, \ldots, n-1 \mathrm{com}|k-l|>1$.

Então a aplicação definida sobre $B_{n}$ por $\sigma_{k} \longmapsto\left(T_{k}\right)_{*}$, se estende para um homomorfismo $\rho: B_{n} \rightarrow \operatorname{Aut}\left(\pi_{1}\left(Y, Q_{0}\right)\right)$, ou seja, contruímos uma representação do grupo de tranças $B_{n}$ nos automorfismos do grupo fundamental de $Y$ com ponto base $Q_{0}$. Agora 
nosso objetivo é mostrar que esta representação $\rho$ é a representação de tipo Artin associada ao $\operatorname{par}\left(\pi_{1}\left(X, P_{0}\right),[\alpha]\right)$.

Considere $Q_{0}=\frac{n+1}{2}-i \frac{n+1}{2}$ e seja $\gamma_{k}:[0,1] \rightarrow Y$ o caminho em $Y$ de $Q_{0}$ a $f_{k}\left(P_{0}\right)$ mostrado na figura 5.4.

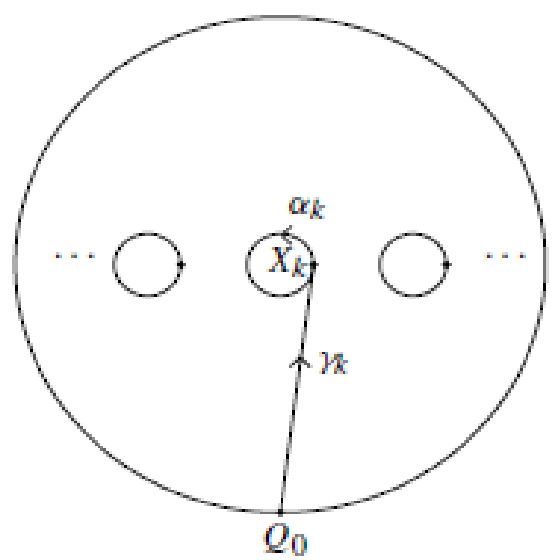

Figura 5.4: Caminho $\gamma_{k}$

Identifique $\pi_{1}\left(Y, Q_{0}\right)$ com $H^{* n}=H_{1} * \cdots * H_{n}$, onde $H=\pi_{1}\left(X, P_{0}\right)$ e $H_{k}=\pi_{1}\left(X_{k}, P_{k}\right)$, para $k=1, \ldots, n$, sendo o $k$-ésimo isomorfismo $\phi_{k}: H \rightarrow H_{i}$ definido por

$$
\phi_{k}([\beta])=\left[\gamma_{k} f_{k}(\beta) \overline{\gamma_{k}}\right]
$$

Temos assim, a seguinte proposição.

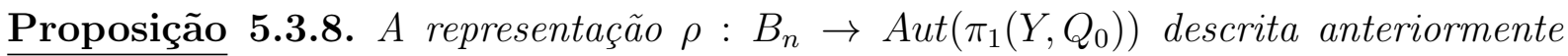
coincide com a representação de tipo Artin de $B_{n}$ associada ao par $\left(\pi_{1}\left(X, P_{0}\right),[\alpha]\right)$.

Demonstração. Seja $\beta$ um caminho em $X$ baseado em $P_{0}$. Primeiro observe, com ajuda dos desenhos, o seguinte:

(a) $T_{k}\left(\gamma_{k} f_{k}(\beta) \overline{\gamma_{k}}\right) \simeq \gamma_{k} \overline{\alpha_{k}} \overline{\gamma_{k}} \gamma_{k+1} f_{k+1}(\beta) \overline{\gamma_{k+1}} \gamma_{k} \alpha_{k} \overline{\gamma_{k}}$ 
Tabela 5.1: Transformação do caminho $\gamma_{k} f_{k}(\beta) \overline{\gamma_{k}}$ sob a aplicação $T_{k}$.

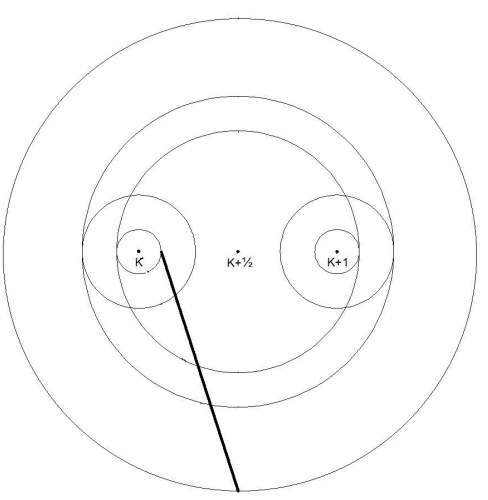

(1)

$\gamma_{k} f_{k}(\beta) \overline{\gamma_{k}}$

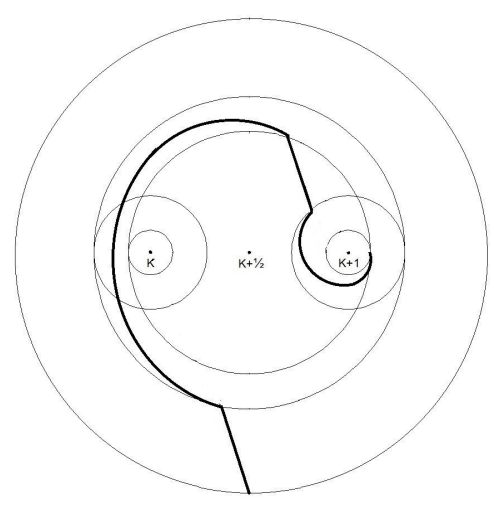

(3)

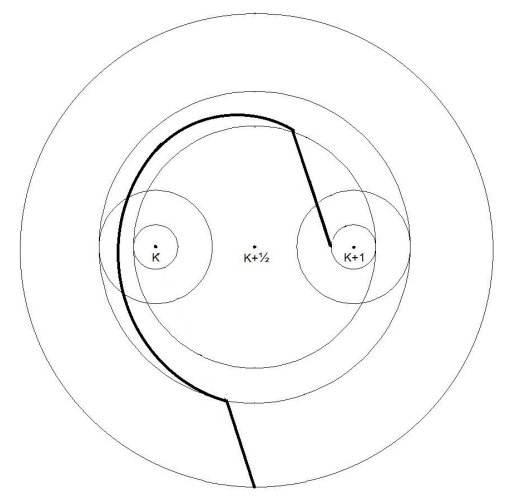

(2)

$T(k+1, \epsilon, 2 \epsilon)^{-1}((2))$

$(4)$

$T_{k}\left(\gamma_{k} f_{k}(\beta) \overline{\gamma_{k}}\right)$

Onde (4) da tabela 5.1 é homotópico ao caminho da figura 5.5. 


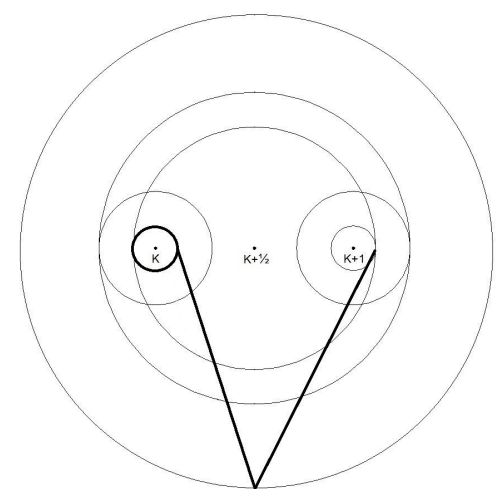

Figura 5.5: $\gamma_{k} \overline{\alpha_{k}} \overline{\gamma_{k}} \gamma_{k+1} f_{k+1}(\beta) \overline{\gamma_{k+1}} \gamma_{k} \alpha_{k} \overline{\gamma_{k}}$

(b) $T_{k}\left(\gamma_{k+1} f_{k+1}(\beta) \overline{\gamma_{k+1}}\right) \simeq \gamma_{k} \alpha_{k} f_{k}(\beta) \overline{\alpha_{k}} \overline{\gamma_{k}}$

Onde (3) da tabela 5.2 é homotópico ao caminho da figura 5.6.

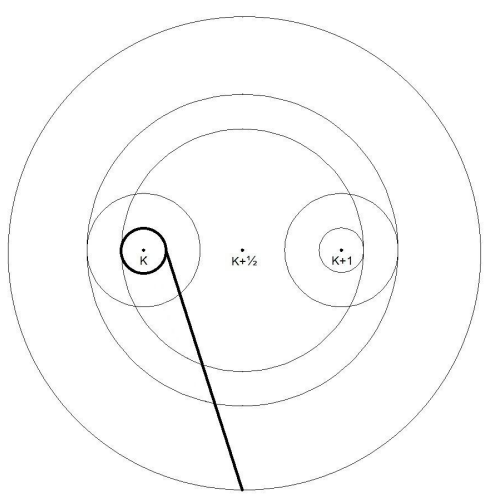

Figura 5.6: $\gamma_{k} \alpha_{k} f_{k}(\beta) \overline{\alpha_{k}} \overline{\gamma_{k}}$ 
Tabela 5.2: Transformação do caminho $\gamma_{k+1} f_{k+1}(\beta) \overline{\gamma_{k+1}}$ sob a aplicação $T_{k}$.

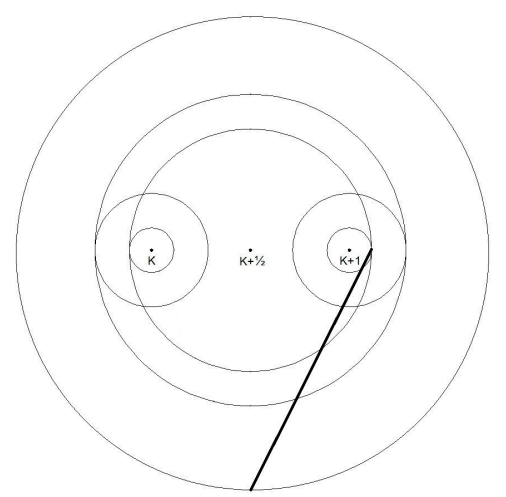

$(1)$

$\gamma_{k+1} f_{k+1}(\beta) \overline{\gamma_{k+1}}$

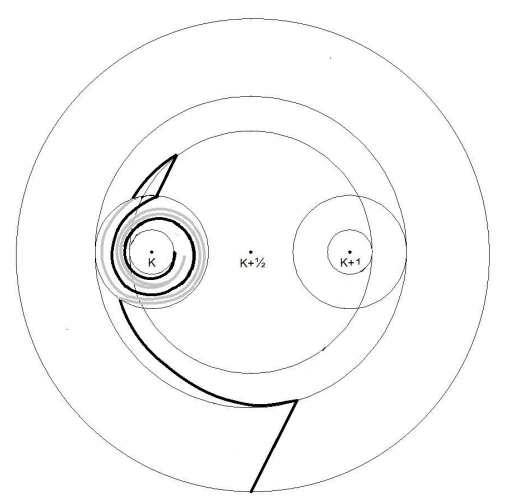

(3)

$T_{k}\left(\gamma_{k+1} f_{k+1}(\beta) \overline{\gamma_{k+1}}\right)$

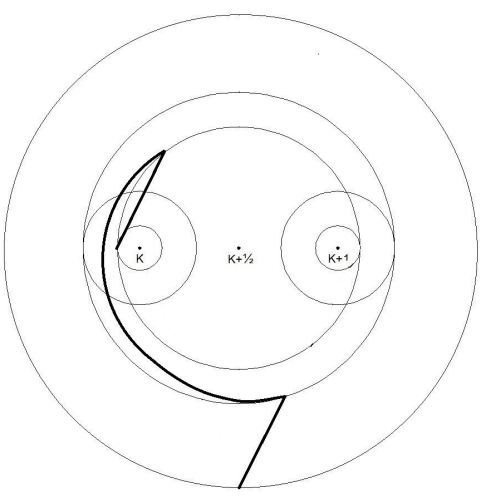

(2)

$T\left(k+\frac{1}{2}, \frac{1}{2}+\epsilon, \frac{1}{2}+2 \epsilon\right)\left(\gamma_{k+1} f_{k+1}(\beta) \overline{\gamma_{k+1}}\right)$

(c) $T_{k}\left(\gamma_{j} f_{j}(\beta) \overline{\gamma_{j}}\right) \simeq \gamma_{j} f_{j}(\beta) \overline{\gamma_{j}}$, para $j \neq k, k+1$.

Lembrando que $\alpha_{k}=f_{k} \circ \alpha$, segue que

$$
\left(T_{k}\right)_{*}:\left\{\begin{array}{rlr}
\phi_{k}([\beta]) & \longmapsto \phi_{k}([\alpha])^{-1} \phi_{k+1}([\beta]) \phi_{k}([\alpha]) \\
\phi_{k+1}([\beta]) & \longmapsto \phi_{k}([\alpha]) \phi_{k}([\beta]) \phi_{k}([\alpha])^{-1} \\
\phi_{j}([\beta]) & \longmapsto \phi_{j}([\beta]) \quad, \text { para } j \neq k, k+1
\end{array}\right.
$$


Agora vamos construir um invariante de enlaçamentos usando a representação $\rho$ da proposição anterior. Vamos associar a um enlaçamento $L$ o espaço $\Omega(L, X)$ obtido mediante uma "cirurgia" sobre $L$. Descrevemos este processo a seguir. Seja $L=K_{1} \cup \cdots \cup K_{m}$ o enlaçamento cujas componentes são os nós $K_{1}, \ldots, K_{m}$, e considere $\left\{T_{i}: D^{2} \times S^{1} \rightarrow S^{3}\right\}_{i=1}^{m}$ o framing preferido de $L$. Denote por $\stackrel{\circ}{T}_{i}$ o interior de $T_{i}\left(D^{2} \times S^{1}\right)$ para $i=1, \ldots, m$. Considere o espaço

$$
\Omega^{\prime}(L)=S^{3} \backslash\left(\bigcup_{i=1}^{m} \stackrel{\circ}{T}_{i}\right)
$$

Considere $m$ cópias $X_{1}, \ldots, X_{m}$ de $X$ e denote por $f_{i}: X \rightarrow X_{i}$ o homeomorfismo natural e escreva $\alpha_{i}=f_{i} \circ \alpha$, para todo $i=1, \ldots, m$. Então definimos

$$
\Omega(L, X)=\left(\Omega^{\prime}(L) \dot{\bigcup}\left(\bigcup_{i=1}^{m}\left(X_{i} \times S^{1}\right)\right)\right) / \sim
$$

onde $\sim$ é a identificação dada por

$$
\left(\alpha_{i}(t), \eta\right) \sim T_{i}\left(e^{2 \pi i t}, \eta\right), \text { para } i=1, \ldots, m, t \in[0,1] \text { e } \eta \in S^{1}
$$

O seguinte teorema fornece uma segunda prova do fato que $\Gamma_{(H, h)}$ é um invariante de enlaçamentos para qualquer grupo finitamente gerado $H$ e qualquer elemento $h \in H$.

Teorema 5.3.9. Seja $\beta$ uma trança e $\widehat{\beta}$ o fecho da trança $\beta$ (ou a trança fechada associada a $\beta$ ). Seja $X$ um complexo $C W$ com ponto base $P_{0}$. Então

$$
\pi_{1}(\Omega(\widehat{\beta}, X)) \simeq \Gamma_{(H, h)}(\beta)
$$

onde $H=\pi_{1}\left(X, P_{0}\right)$ e $h=[\alpha]$, onde $\alpha$ é um laço em $X$ baseado em $P_{0}$.

Demonstração. Usemos a decomposição de $S^{3}$ em duas copias $T_{1}$ e $T_{2}$ do toro sólido $\mathbf{D} \times \mathbf{S}^{\mathbf{1}}$, de maneira que

$$
S^{3}=T_{2} \cup_{\kappa} T_{1}
$$

onde $\kappa: \partial T_{1} \rightarrow \partial T_{2}$ é um homeomorfismo tal que $\kappa(\partial \mathbf{D})=S^{1}$ e $\kappa\left(S^{1}\right)=\partial \mathbf{D}$ (veja [6]).

Seja $g: T_{1} \rightarrow S^{3}$ a função inclusão e seja $f: \mathbf{D} \times[0,1] \rightarrow T_{1}=\mathbf{D} \times S^{1}$ a aplicação 
identificação definida por

$$
f(p, t)=\left(p, e^{2 \pi i t}\right) \text { para } p \in \mathbf{D} \text { e } t \in[0,1]
$$

Note que de fato $f$ é uma identificação pois $f$ é sobrejetora, $\mathbf{D} \times[0,1]$ é compacto e $T_{1}$ é espaço de Hausdorff (proposição 3.1.13).

A trança fechada $\widehat{\beta}$ é o enlaçamento orientado que é visto como a composição da trança $\beta:\{1, \ldots, n\} \times[0,1] \rightarrow D \times[0,1]$ (tal como vimos no capítulo 4 ) com a aplicação $g \circ f: \mathbf{D} \times[0,1] \rightarrow S^{3}$. A orientação de $\widehat{\beta}$ é induzida pela orientação do intervalo $[0,1]$, como se mostra na figura 5.1. Agora vamos produzir um framing $\Lambda$ de $\widehat{\beta}$ pela escolha de uma longitude $\lambda_{i}$ para cada componente $K_{i}$ de $\widehat{\beta}$ cuja projeção é indicada na figura 5.7 nas proximidades dos cruzamentos. É fácil ver, pela contagem dos cruzamentos, que este é o framing preferido de $\widehat{\beta}$.

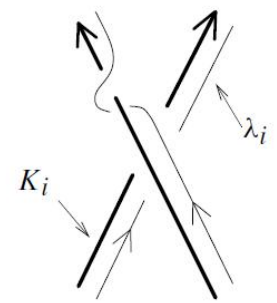

$\sigma_{k}$

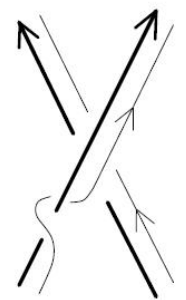

$\sigma_{k}^{-1}$

Figura 5.7: Escolhendo um framing para $\widehat{\beta}$.

Escreva $\beta=\sigma_{k_{1}}^{\epsilon_{1}} \ldots \sigma_{k_{r}}^{\epsilon_{r}}$ e defina $T_{\beta}^{\mathbf{D}}: \mathbf{D} \rightarrow \mathbf{D}$ por

$$
T_{\beta}^{\mathbf{D}}=\left(T_{k_{1}}^{\mathbf{D}}\right)^{\epsilon_{1}} \circ \cdots \circ\left(T_{k_{r}}^{\mathbf{D}}\right)^{\epsilon_{r}}
$$

Analogamente, defina $T_{\beta}: Y \rightarrow Y$ por

$$
T_{\beta}=T_{k_{1}}^{\epsilon_{1}} \circ \cdots \circ T_{k_{r}}^{\epsilon_{r}}
$$

Para $j=1, \ldots, n$, escolha $b_{j}=j+\epsilon \in \partial D(j, \varepsilon)$ como ponto base do espaço $X_{j}$ quando este é adjuntado a $\mathbf{D}$ mediante a identificação (5.3.3).

Pelo corolário $5.3 .5, T_{k_{j}}^{\mathbf{D}}$ é isotópica à $I d_{\mathbf{D}}$ relativamente a $\partial \mathbf{D}$, logo pela observação 
3.4.6, temos que $T_{\beta}^{\mathbf{D}}$ é isotópico à $I d_{\mathbf{D}}$ relativamente a $\partial \mathbf{D}$. Portanto, existe um homeomorfismo

$$
U: \mathbf{D} \times[0,1] \rightarrow \mathbf{D} \times[0,1]
$$

tal que $U(x, 0)=(x, 0)$ e $U(x, 1)=\left(T_{\beta}^{\mathbf{D}}(x), 1\right)$, para todo $x$ em D. Além disso, $U$ fixa $\partial \mathbf{D} \times[0,1]$ ponto a ponto.

Observe que $U$ aplica o conjunto

$$
\bigcup_{j=1}^{n} D(j, \epsilon) \times[0,1]
$$

numa vizinhança tubular (do representante) da trança $\beta$, e $g \circ f \circ U$ aplica $\left\{b_{j} \times[0,1]\right.$ : $j=1, \ldots, n\}$ no framing da trança fechada $\widehat{\beta}$ equivalente áquele descrito na figura 5.7 , a saber o framing preferido.

Portanto, o espaço $\Omega(\widehat{\beta}, X)$ é homeomorfo a $T_{1}^{\prime} \cup T_{2}$ onde

$$
T_{1}^{\prime}=Y \times[0,1] /\left((x, 0) \sim\left(T_{\beta}(y), 1\right)\right)
$$

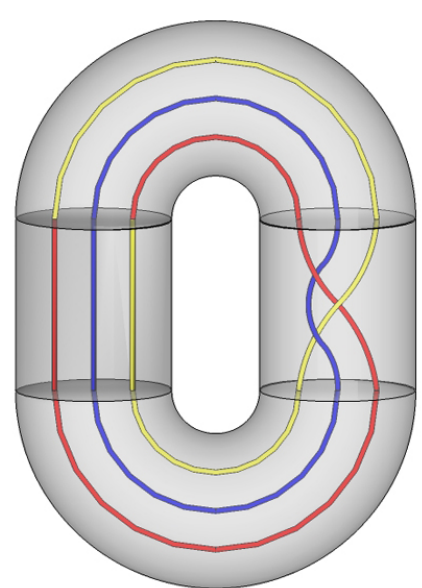

Figura 5.8: Exemplo do espaço $T_{1}^{\prime}$

Pelo exemplo 3.5.11 do capítulo 3, temos (a extensão HNN):

$$
\pi_{1}\left(T_{1}^{\prime}\right) \simeq \pi_{1}\left(Y, Q_{0}\right) *\langle t\rangle /\left\langle t^{-1} x t=\left(T_{\beta}\right)_{*}(x): x \in \pi_{1}\left(Y, Q_{0}\right)\right\rangle^{N}
$$


com $t$ de ordem infinita e onde $N=\pi_{1}\left(Y, Q_{0}\right) *\langle t\rangle$. Assim,

$$
\pi_{1}\left(T_{1}^{\prime}\right) \simeq \pi_{1}\left(Y, Q_{0}\right) *\langle t\rangle /\left\langle t^{-1} x t=\rho(\beta)(x): x \in \pi_{1}\left(Y, Q_{0}\right)\right\rangle^{N}
$$

Quando adjuntamos $T_{1}^{\prime}$ a $T_{2}$ o gerador $t$ de $\pi_{1}\left(T_{1}^{\prime}\right)$ é anulado da mesma maneira como são anulados os geradores $m$ e $l$ de $\pi_{1}\left(S^{1} \times S^{1}\right)$. Portanto, do corolário 3.5.3 e usando a notação deste capítulo, obtemos

$$
\pi_{1}(\Omega(\widehat{\beta}, X)) \simeq H^{* n} /\left\langle x=\rho(\beta)(x): x \in H^{* n}\right\rangle^{H^{* n}}=\Gamma_{(H, h)}(\beta)
$$

\subsection{Fidelidade da Representação de Tipo Artin}

Nesta seção pretendemos demonstrar a fidelidade da representação de Artin para o caso em que o elemento $h$ é não trivial, pois como já vimos no exemplo 5.1.3, para $h$ trivial $\rho$ tem como kernel ao grupo das tranças puras.

Definição 5.4.1. Sejam $G$ e F grupos tal que $G$ é representado em Aut $(F)$, isto é, existe uma representação (homomorfismo) $\zeta: G \rightarrow$ Aut $(F)$. Dizemos que a representação $\zeta$ é fiel se for injetora, isto é, se $\zeta(g)=I d_{F}$ então $g=e_{G}$.

Relembramos que para um grupo $H$ e um inteiro $n$ positivo, denotamos por $H^{* n}$ o produto livre $H_{1} * \cdots * H_{n}$, onde cada fator livre $H_{i}$ é uma copia de $H$ mediante um isomorfismo dado e denotado por $\phi_{i}: H \rightarrow H_{i}$. Nosso objetivo então é demonstrar a seguinte proposição.

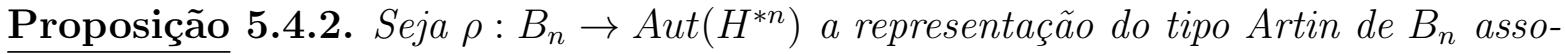
ciada ao par $(H, h)$.

(1) $S e h \neq e_{H}$ então $\rho$ é fiel.

(2) Se $h=e_{H}$ então $\operatorname{ker}(\rho)$ é o grupo das tranças puras e $B_{n} / \operatorname{Ker}(\rho) \simeq \Sigma_{n}$, onde $\Sigma_{n}$ atúa por permutação dos fatores livres de $H^{* n}$, respeitando os isomorfismos $\phi_{1}, \ldots, \phi_{n}$. 
Vamos precisar de algumas ferramentas para demonstrar a proposição anterior.

A primeira ferramenta é a seguinte.

Proposição 5.4.3 (Dehornoy: ver [9], [10]). Seja $B_{[2, n]}$ o subgrupo de $B_{n}$ gerado por $\sigma_{2}, \ldots, \sigma_{n-1}$. Seja $\beta$ em $B_{n}$. Então, ou $\beta$ está em $B_{[2, n]}$, ou, uma das tranças $\beta$ ou $\beta^{-1}$ pode ser escrito como

$$
\alpha_{0} \sigma_{1} \alpha_{1} \sigma_{1} \ldots \sigma_{1} \alpha_{l}
$$

onde $l \geq 1$ e $\alpha_{0}, \ldots, \alpha_{l} \in B_{[2, n]}$.

A segunda ferramenta é um lema. Considere $h \neq e_{H}$ em $H$ fixo e escreva $h_{i}=\phi_{i}(h)$, para $i=1, \ldots, n$.

Lema 5.4.4. Seja $K=H_{1} * \cdots * H_{n}$. Seja $\mu$ em $H^{* n}$ tal que a forma normal de $\mu$ respeito $\grave{a}$ decomposição $H^{* n}=H_{1} * K$ começa com $h_{1}^{-1}$ e termina com $h_{1}$. Então:

(1) A forma normal de $\rho\left(\sigma_{1}\right)(\mu)$ com respeito à decomposição $H^{* n}=H_{1} * K$ também começa com $h_{1}^{-1}$ e termina com $h_{1}$.

(2) Seja $k \in\{2, \ldots, n-1\}$ e $\in \in\{ \pm 1\}$. A forma normal de $\rho\left(\sigma_{k}^{\epsilon}\right)(\mu)$ com respeito à decomposição $H^{* n}=H_{1} * K$ também começa com $h_{1}^{-1}$ e termina com $h_{1}$.

Demonstração. Seja $v$ um elemento não trivial de $H_{1} * H_{2}$. Pelo teorema da forma normal (teorema 1.4.5) podemos supor que $v$ pode ser escrito como

$$
v=\phi_{1}\left(x_{1}\right) \phi_{2}\left(y_{1}\right) \ldots \phi_{1}\left(x_{l}\right) \phi_{2}\left(y_{l}\right)
$$

onde $x_{1}, \ldots, x_{l}, y_{1}, \ldots, y_{l}$ são elementos não triviais de $H$ e $l \geq 1$. Aplicando o automorfismo $\rho\left(\sigma_{1}\right)$ a $v$ obtemos

$$
\begin{aligned}
\rho\left(\sigma_{1}\right)(v) & =h_{1}^{-1} \phi_{2}\left(x_{1}\right) h_{1} h_{1} \phi_{1}\left(y_{1}\right) h_{1}^{-1} \ldots h_{1}^{-1} \phi_{2}\left(x_{l}\right) h_{1} h_{1} \phi_{1}\left(y_{l}\right) h_{1}^{-1} \\
& =h_{1}^{-1} \phi_{2}\left(x_{1}\right) h_{1}^{2} \phi_{1}\left(y_{1}\right) h_{1}^{-2} \ldots h_{1}^{-2} \phi_{2}\left(x_{l}\right) h_{1}^{2} \phi_{1}\left(y_{l}\right) h_{1}^{-1}
\end{aligned}
$$

Portanto, a forma normal de $\rho\left(\sigma_{1}(v)\right)$ começa com $h_{1}^{-1}$. Analogamente se demonstra que se a forma normal de $v$ for

$$
v=\phi_{2}\left(y_{1}\right) \phi_{1}\left(x_{1}\right) \ldots \phi_{2}\left(y_{l}\right) \phi_{1}\left(x_{l}\right)
$$


onde $x_{1}, \ldots, x_{l}, y_{1}, \ldots, y_{l}$ são elementos não triviais de $H$ e $l \geq 1$, então $\rho\left(\sigma_{1}\right)(v)$ termina $\operatorname{com} h_{1}$.

Agora, suponha que a forma normal de $\mu$ com respeito à decomposição

$$
H^{* n}=\left(H_{1} * H_{2}\right) *\left(H_{3} * \cdots * H_{n}\right)
$$

é da forma

$$
\mu=v_{0} w_{1} v_{1} \ldots w_{l} v_{l}
$$

onde $v_{i}$ é um elemento não nulo de $H_{1} * H_{2}$ e $w_{i}$ é um elemento não nulo de $H_{3} * \cdots * H_{n}$, para $i=1, \ldots, l$, e $l \geq 0$. Note que esta forma normal de $\mu$ está certa, pois se for da forma $v_{0} w_{1} v_{1} \ldots w_{l}$ ou da forma $w_{1} v_{1} \ldots w_{l} v_{1}$, significaria que $w_{1}$ começa com $h_{1}^{-1}$, ou que $w_{l}$ termina com $h_{1}$, o que é uma contradição. Observe que $v_{0}$ começa com $h_{1}^{-1}$ e que $v_{l}$ termina com $h_{1}$ pois $\mu$ começa com $h_{1}^{-1}$ e termina com $h_{1}$. Além disso, note que $\rho\left(\sigma_{1}\right)$ deixa invariante o conjunto $H_{1} * H_{2}$ e que $\rho\left(\sigma_{1}\right)$ é a identidade sobre $H_{3} * \cdots * H_{n}$. Portanto temos

$$
\rho\left(\sigma_{1}\right)(\mu)=\rho\left(\sigma_{1}\right)\left(v_{0}\right) w_{1} \rho\left(\sigma_{1}\right)\left(v_{1}\right) \ldots w_{l} \rho\left(\sigma_{1}\right)\left(v_{l}\right)
$$

Pelas observações feitas $\rho\left(\sigma_{1}\right)\left(v_{0}\right)$ começa com $h_{1}^{-1}$ e $\rho\left(\sigma_{1}\right)\left(v_{l}\right)$ termina com $h_{1}$ com respeito à decomposição $H^{* n}=H_{1} * K$. Isto demonstra a parte (1).

Para provar a parte (2), considere $k \in\{2, \ldots, n-1\}$ e $\epsilon \in\{ \pm 1\}$. Escreva

$$
\mu=h_{1}^{-1} w_{1} v_{1} \ldots v_{l-1} w_{l} h_{1}
$$

com $v_{1}, \ldots, v_{l-1}$ elementos de $H_{1}$ não nulos e $w_{1}, \ldots, w_{l} \in K$ também não nulos. Note que $\rho\left(\sigma_{k}^{\epsilon}\right)$ é a identidade em $H_{1}$ e que $K$ fica invariante sob a aplicação $\rho\left(\sigma_{k}^{\epsilon}\right)$. Logo temos

$$
\rho\left(\sigma_{k}^{\epsilon}\right)(\mu)=h_{1}^{-1} \rho\left(\sigma_{k}^{\epsilon}\right)\left(w_{1}\right) v_{1} \ldots v_{l-1} \rho\left(\sigma_{k}^{\epsilon}\right)\left(w_{l}\right) h_{1}
$$

Ficando demonstrada a parte (2).

Já estamos em condições de demonstrar a proposição 5.4.2.

Demonstração proposição 5.4.2. Vamos demonstrar só a parte (1) pois a parte (2) já foi 
vista no exemplo 5.1.3. Vamos fazer indução sobre o número $n$. Suponha $n=2$. Fazendo um cálculo simples e usando indução sobre o número $l$, obtemos

$$
\begin{aligned}
\rho\left(\sigma_{1}^{2 l}\right)\left(h_{1}\right) & =\left(h_{2} h_{1}\right)^{-l} h_{1}\left(h_{2} h_{1}\right)^{l} \quad, \text { para todo } l \in \mathbb{Z} \backslash\{0\} \\
\rho\left(\sigma_{1}^{2 l+1}\right)\left(h_{1}\right) & =\left(h_{2} h_{1}\right)^{-l} h_{1}^{-1} h_{2} h_{1}\left(h_{2} h_{1}\right)^{l},
\end{aligned}
$$

Note que se $\rho\left(\sigma_{1}^{2 l}\right)=h_{1}$, para algum inteiro $l$ não nulo , então teríamos uma contradição com o teorema da forma normal para produtos livres (teorema 1.4.5). Portanto,

$$
\rho\left(\sigma_{1}^{2 l}\right)\left(h_{1}\right) \neq h_{1}
$$

para todo inteiro $l$ não nulo. Analogamente, $\rho\left(\sigma_{1}^{2 l+1}\right)\left(h_{1}\right) \neq h_{1}$, para todo inteiro $l$. Portanto, $\rho: B_{2} \rightarrow \operatorname{Aut}\left(H_{1} * H_{2}\right)$ satisfaz que $\rho(\beta) \neq I d_{H_{1} * H_{2}}$ para toda 2-trança $\beta$ não trivial, ou seja, $\rho$ é fiel.

Suponha $n \geq 3$ e que se verifica o resultado para inteiros positivos $m$ tais que $2 \leq m \leq$ $n-1$. Seja $\beta$ uma trança não trivial em $B_{n}$, pela proposição 5.4.3, ou $\beta$ é um elemento de $B_{[2, n]}$, ou, uma das tranças $\beta$ ou $\beta^{-1}$ se escreve como um produto

$$
\alpha_{0} \sigma_{1} \alpha_{1} \ldots \sigma_{1} \alpha_{l}
$$

onde $\alpha_{0}, \ldots, \alpha_{l}$ são elementos de $B_{[2, n]}$, para algum inteiro $l \geq 1$.

Se $\beta$ está em $B_{[2, n]}$ então pela hipótese de indução, $\rho(\beta)$ não é a identidade sobre $K=H_{2} * \cdots * H_{n}$. Logo, $\rho(\beta)$ não é a identidade de $H^{* n}=H_{1} * K$.

Suponha $\beta=\alpha_{0} \sigma_{1} \alpha_{1} \ldots \sigma_{1} \alpha_{l}$. Observe que $\rho\left(\sigma_{1} \alpha_{l}\right)\left(h_{1}\right)=h_{1}^{-1} h_{2} h_{1}$ e portanto,

$$
\begin{aligned}
\rho(\beta)\left(h_{1}\right) & =\rho\left(\alpha_{0} \sigma_{1} \alpha_{1} \ldots \sigma_{1} \alpha_{l}\right)\left(h_{1}\right) \\
& =\rho\left(\alpha_{0} \sigma_{1} \alpha_{1} \ldots \alpha_{l-1}\right) \circ \rho\left(\sigma_{1} \alpha_{l}\right)\left(h_{1}\right) \\
& =\rho\left(\alpha_{0} \sigma_{1} \alpha_{1} \ldots \alpha_{l-1}\right)\left(h_{1}^{-1} h_{2} h_{1}\right)
\end{aligned}
$$

pelo lema 5.4.4, $\rho(\beta)\left(h_{1}\right)$ começa com $h_{1}^{-1}$ e acaba com $h_{1}$. Assim, $\rho(\beta)\left(h_{1}\right) \neq h_{1}$ (a igualdade contradiz o teorema da forma normal para produtos livres). Portanto $\rho(\beta) \neq$ $I d_{H^{* n}}$. 
Finalmente, se $\beta^{-1}=\alpha_{0} \sigma_{1} \alpha_{1} \ldots \sigma_{1} \alpha_{l}$, então pelo caso anterior, $\rho\left(\beta^{-1}\right) \neq I d_{H^{* n}}$, ou seja, $\rho(\beta)^{-1} \neq I d_{H^{* n}}$, e portanto, $\rho(\beta) \neq I d_{H^{* n}}$.

\subsection{Alguns Exemplos}

Nesta seção vamos fazer alguns cálculos para exemplificar o modo de obter o grupo associado a um enlaçamento (ou nó) do presente capítulo. Seja $\rho: B_{n} \rightarrow \operatorname{Aut}\left(H^{* n}\right)$ a representação tipo Artin de $B_{n}$. Lembramos que $H^{* n}$ denota o produto livre de $n$ cópias $H_{1}, \ldots, H_{n}$ de um grupo $H$ arbitrário, que $h$ é um elemento arbitrário fixo em $H$, e que $\phi_{k}: H \rightarrow H_{k}$ é um isomorfismo, para $k=1, \ldots, n$. Denotaremos por $y_{k}$ o elemento no $k$-ésimo fator livre $H_{k}$ de $H^{* n}$ que é imagem de um elemento $y$ em $H$ sob o isomorfismo $\phi_{k}$. Com estas notações temos que o automorfismo $\tau_{k}$ de $H^{* n}$ fica definido por

$$
\tau_{k}:\left\{\begin{aligned}
y_{k} & \longmapsto h_{k}^{-1} y_{k+1} h_{k} \\
y_{k+1} & \longmapsto h_{k} y_{k} h_{k}^{-1} \\
y_{j} & \longmapsto y_{j} \quad, \text { se } j \neq k, k+1
\end{aligned}\right.
$$

e seu inverso por

$$
\tau_{k}^{-1}:\left\{\begin{array}{rl}
y_{k} & \longmapsto h_{k+1}^{-1} y_{k+1} h_{k+1} \\
y_{k+1} & \longmapsto h_{k+1} y_{k} h_{k+1}^{-1} \\
y_{j} & \longmapsto y_{j}
\end{array}, \text { se } j \neq k, k+1\right.
$$

Temos algumas observações óbvias:

$$
\begin{array}{ll}
\tau_{k}\left(h_{k}\right)=h_{k}^{-1} h_{k+1} h_{k} ; & \tau_{k}\left(h_{k+1}\right)=h_{k} \\
\tau_{k}^{-1}\left(h_{k}\right)=h_{k+1} ; & \tau_{k}^{-1}\left(h_{k+1}\right)=h_{k+1} h_{k} h_{k+1}^{-1}
\end{array}
$$

Usaremos o método dado em [3], pag. 73, para encontrar a trança cujo fecho é o enlaçamento (ou nó) em estudo.

Considere o nó trevo $T$.

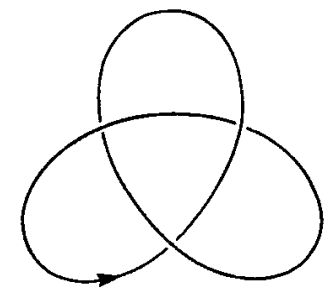

Figura 5.9: O nó Trevo T. 
É fácil verificar que $T=\widehat{\sigma_{1}^{-3}}$. Portanto, $\rho: B_{2} \rightarrow \operatorname{Aut}\left(H^{* 2}\right)$ satisfaz $\rho\left(\sigma_{1}^{-3}\right)=\tau_{1}^{-3}$. Temos para $y_{1}$ em $H_{1}$

$$
\begin{aligned}
\tau_{1}^{-3}\left(y_{1}\right) & =\tau_{1}^{-2}\left(h_{2}^{-1} y_{2} h_{2}\right) \\
& =\tau_{1}^{-1}\left(h_{2} h_{1}^{-1} h_{2}^{-1} h_{2} y_{1} h_{2}^{-1} h_{2} h_{1} h_{2}^{-1}\right) \\
& =\tau_{1}^{-1}\left(h_{2} h_{1}^{-1} y_{1} h_{1} h_{2}^{-1}\right) \\
& =h_{2} h_{1} h_{2}^{-1} h_{2}^{-1} h_{2}^{-1} y_{2} h_{2} h_{2} h_{2} h_{2} h_{1}^{-1} h_{2}^{-1} \\
& =h_{2} h_{1} h_{2}^{-3} y_{2} h_{2}^{3} h_{1}^{-1} h_{2}^{-1}
\end{aligned}
$$

e para $y_{2}$ em $H_{2}$

$$
\begin{aligned}
\tau_{1}^{-3}\left(y_{2}\right) & =\tau_{1}^{-2}\left(h_{2} y_{1} h_{2}^{-1}\right) \\
& =\tau_{1}^{-1}\left(h_{2} h_{1} h_{2}^{-1} h_{2}^{-1} y_{2} h_{2} h_{2} h_{1}^{-1} h_{2}^{-1}\right) \\
& =\tau_{1}^{-1}\left(h_{2} h_{1} h_{2}^{-2} y_{2} h_{2}^{2} h_{1}^{-1} h_{2}^{-1}\right) \\
& =h_{2} h_{1} h_{2}^{-1} h_{2} h_{2} h_{1}^{-2} h_{2}^{-1} h_{2} y_{1} h_{2}^{-1} h_{2} h_{1}^{2} h_{2}^{-1} h_{2} h_{1}^{2} h_{2}^{-1} h_{2}^{-1} h_{2} h_{1}^{-1} h_{2}^{-1} \\
& =h_{2} h_{1} h_{2} h_{1}^{-2} y_{1} h_{1}^{2} h_{2}^{-1} h_{1}^{-1} h_{2}^{-1}
\end{aligned}
$$

Portanto, $\Gamma(T)$ é quociente de $H_{1} * H_{2}$ pelas relações

- $y_{1}=h_{2} h_{1} h_{2}^{-3} y_{2} h_{2}^{3} h_{1}^{-1} h_{2}^{-1}$

- $y_{2}=h_{2} h_{1} h_{2} h_{1}^{-2} y_{1} h_{1}^{2} h_{2}^{-1} h_{1}^{-1} h_{2}^{-1}$

para todo $y_{k} \in H_{k}$, com $k=1,2$. Como estas relações valem para todo elemento de $H_{1}$ e $H_{2}$, temos em particular

$$
h_{1}=h_{2} h_{1} h_{2}^{3} h_{2} h_{2}^{3} h_{1}^{-1} h_{2}^{-1} \Leftrightarrow h_{1} h_{2} h_{1}=h_{2} h_{1} h_{2}
$$

e

$$
h_{2}=h_{2} h_{1} h_{2} h_{1}^{-2} h_{1} h_{1}^{2} h_{2}^{-1} h_{1}^{-1} h_{2}^{-1} \Leftrightarrow h_{1} h_{2} h_{1}=h_{2} h_{1} h_{2}
$$

Usando (1) em $y_{1}=\tau_{1}^{-3}\left(y_{1}\right)$ obtemos $y_{1}=h_{1}^{-3} h_{2} h_{1} y_{2} h_{2}^{3} h_{1}^{-1} h_{2}^{-1}$, que é equivalente a $h_{1}^{3} y_{1} h_{2} h_{1}=h_{2} h_{1} y_{2} h_{2}^{3}$. Analogamente, usando (1) em $y_{2}=\tau_{1}^{-3}\left(y_{2}\right)$ obtemos $y_{2}=h_{2}^{-1} h_{1} h_{2} y_{1} h_{1} h_{2}^{-1} h_{1}^{-1}$ e portanto, $h_{2} y_{2} h_{1} h_{2}=h_{1} h_{2} y_{1} h_{1}$. Assim, $\Gamma(T)$ é quociente de $H_{1} * H_{2}$ pelas relações

(1) $h_{1} h_{2} h_{1}=h_{2} h_{1} h_{2}$

(2) $h_{1}^{3} y_{1} h_{2} h_{1}=h_{2} h_{1} y_{2} h_{2}^{3}$

(3) $h_{2} y_{2} h_{1} h_{2}=h_{1} h_{2} y_{1} h_{1}$

para todo $y_{k} \in H_{k}$, com $k=1,2$. 
Agora vamos estudar um exemplo um pouco mais complexo. Seja $M$ o nó Granny (ver figura 5.10).

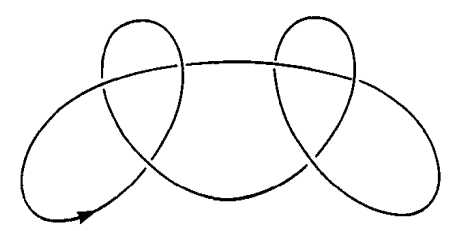

Figura 5.10: O nó Granny.

Usando o método de [3], pag. 73, obtemos que

$$
M=\widehat{\beta} \text { onde } \beta=\sigma_{2}^{-3} \sigma_{1}^{-3}
$$

Aqui $\beta$ é uma 3-trança e portanto $\rho: B_{3} \rightarrow A u t\left(H^{* 3}\right)$ aplica $\beta \longmapsto \tau_{2}^{-3} \circ \tau_{1}^{-3}$. Sabemos pelo trabalho feito anteriormente que

$$
\tau_{1}^{-3}:\left\{\begin{array}{lll}
y_{1} & \longmapsto & h_{2} h_{1} h_{2}^{-3} y_{2} h_{2}^{3} h_{1}^{-1} h_{2}^{-1} \\
y_{2} & \longmapsto & h_{2} h_{1} h_{2} h_{1}^{-2} y_{1} h_{1}^{2} h_{2}^{-1} h_{1}^{-1} h_{2}^{-1} \\
y_{3} & \longmapsto & y_{3}
\end{array}\right.
$$

Agora vamos aplicar $\tau_{2}^{-3}$ e assim calcularemos $\Gamma(M)$. Temos,

$$
\begin{aligned}
\tau_{2}^{-3} \circ \tau_{1}^{-3}\left(y_{1}\right) & =\tau_{2}^{-3}\left(h_{2} h_{1} h_{2}^{-3} y_{2} h_{2}^{3} h_{1}^{-1} h_{2}^{-1}\right) \\
& =\tau_{2}^{-2}\left(h_{3} h_{1} h_{3}^{-3} h_{3}^{-1} y_{3} h_{3} h_{3}^{3} h_{1}^{-1} h_{3}^{-1}\right) \\
& =\tau_{2}^{-2}\left(h_{3} h_{1} h_{3}^{-4} y_{3} h_{3}^{4} h_{1}^{-1} h_{3}^{-1}\right) \\
& =\tau_{2}^{-1}\left(h_{3} h_{2} h_{3}^{-1} h_{1} h_{3} h_{2}^{-4} h_{3}^{-1} h_{3} y_{2} h_{3}^{-1} h_{3} h_{2}^{4} h_{4}^{-1} h_{1}^{-1} h_{3} h_{2}^{-1} h_{3}^{-1}\right) \\
& =\tau_{2}^{-1}\left(h_{3} h_{2} h_{3}^{-1} h_{1} h_{3} h_{2}^{-4} y_{2} h_{2}^{4} h_{3}^{-1} h_{1}^{-1} h_{3} h_{2}^{-1} h_{3}^{-1}\right) \\
& =h_{3} h_{2} h_{3}^{-1} h_{3} h_{3} h_{2}^{-1} h_{3}^{-1} h_{1} h_{3} h_{2} h_{3}^{-1} h_{3}^{-4} h_{3}^{-1} y_{3} h_{3} h_{3}^{4} h_{3} h_{2}^{-1} h_{3}^{-1} h_{1}^{-1} h_{3} h_{2} h_{3}^{-1} h_{3}^{-1} h_{3} h_{2}^{-1} h_{3}^{-1} \\
& =h_{3} h_{2} h_{3} h_{2}^{-1} h_{3}^{-1} h_{1} h_{3} h_{2} h_{3}^{-6} y_{3} h_{3}^{6} h_{2}^{-1} h_{3}^{-1} h_{1}^{-1} h_{3} h_{2} h_{3}^{-1} h_{2}^{-1} h_{3}^{-1}
\end{aligned}
$$

e

$$
\begin{aligned}
\tau_{2}^{-3} \circ \tau_{1}^{-3}\left(y_{2}\right)= & \tau_{2}^{-3}\left(h_{2} h_{1} h_{2} h_{1}^{-2} y_{1} h_{1}^{2} h_{2}^{-1} h_{1}^{-1} h_{2}^{-1}\right) \\
= & \tau_{2}^{-2}\left(h_{3} h_{1} h_{3} h_{1}^{-2} y_{1} h_{1}^{2} h_{3}^{-1} h_{1}-1 h_{3}^{-1}\right) \\
= & \tau_{2}^{-1}\left(h_{3} h_{2} h_{3}^{-1} h_{1} h_{3} h_{2} h_{3}^{-1} h_{1}^{-2} y_{2} h_{1}^{2} h_{3} h_{2}^{-1} h_{3}^{-1} h_{1}^{-1} h_{3} h_{2}^{-1} h_{3}^{-1}\right) \\
= & h_{3} h_{2} h_{3}^{-1} h_{3} h_{3} h_{2}^{-1} h_{3}^{-1} h_{1} h_{3} h_{2} h_{3}^{-1} h_{3} h_{3} h_{2}^{-1} h_{3}^{-1} h_{1}^{-2} y_{1} \\
& h_{1}^{2} h_{3} h_{2} h_{3}^{-1} h_{3}^{-1} h_{3} h_{2}^{-1} h_{3}^{-1} h_{1}^{-1} h_{3} h_{2} h_{3}^{-1} h_{3}^{-1} h_{3} h_{2}^{-1} h_{3}^{-1} \\
= & h_{3} h_{2} h_{3} h_{2}^{-1} h_{3}^{-1} h_{1} h_{3} h_{2} h_{3} h_{2}^{-1} h_{3}^{-1} h_{1}^{-2} y_{1} h_{1}^{2} h_{3} h_{2} h_{3}^{-1} h_{2}^{-1} h_{3}^{-1} h_{1}^{-1} h_{3} h_{2} h_{3}^{-1} h_{2}^{-1} h_{3}^{-1}
\end{aligned}
$$


$\mathrm{e}$

$$
\begin{aligned}
\tau_{2}^{-3} \circ \tau_{1}^{-3}\left(y_{3}\right) & =\tau_{2}^{-3}\left(y_{3}\right) \\
& =\tau_{2}^{-2}\left(h_{3} y_{2} h_{3}^{-1}\right) \\
& =\tau_{2}^{-1}\left(h_{3} h_{2} h_{3}^{-1} h_{3}^{-1} y_{3} h_{3} h_{3} h_{2}^{-1} h_{3}^{-1}\right) \\
& =\tau_{2}^{-1}\left(h_{3} h_{2} h_{3}^{-2} y_{3} h_{3}^{2} h_{2}^{-1} h_{3}^{-1}\right) \\
& =h_{3} h_{2} h_{3}^{-1} h_{3} h_{3} h_{2}^{-2} h_{3}^{-1} h_{3} y_{2} h_{3}^{-1} h_{3} h_{2}^{2} h_{3}^{-1} h_{3}^{-1} h_{3} h_{2}^{-1} h_{3}^{-1} \\
& =h_{3} h_{2} h_{3} h_{2}^{-2} y_{2} h_{2}^{2} h_{3}^{-1} h_{2}^{-1} h_{3}^{-1}
\end{aligned}
$$

Agora é possível determinar $\Gamma(M)$, mas primeiro estudemos as relações $h_{i}=\rho(\beta) h_{i}$, para $i=1,2,3$., pois assim poderemos simplificar tais expressões. Temos $h_{3}=\rho(\beta) h_{3}$, equivalente a

$$
h_{3}=h_{3} h_{2} h_{3} h_{2}^{-2} h_{2} h_{2}^{2} h_{3}^{-1} h_{2}^{-1} h_{3}^{-1}
$$

e portanto, $h_{2} h_{3} h_{2}=h_{3} h_{2} h_{3}$, relação que será denotada por (2). Usando (2) em $h_{1}=$ $\rho(\beta) h_{1}$ obtemos que essas relações são ambas equivalentes a $h_{1} h_{2} h_{1}=h_{2} h_{1} h_{2}$, relação que denotaremos por (1). Usando (1) e $(2)$ em $y_{1}=\rho(\beta) y_{1}$ temos

$$
\begin{aligned}
y_{1} & =h_{2} h_{3} h_{2} h_{2}^{-1} h_{3}^{-1} h_{1} h_{3} h_{2} h_{3}^{-6} y_{3} h_{3}^{6} h_{2}^{-1} h_{3}^{-1} h_{1}^{-1} h_{3} h_{2} h_{2}^{-1} h_{3}^{-1} h_{2}^{-1} \\
& =h_{2} h_{1} h_{3} h_{2} h_{3}^{-6} y_{3} h_{3}^{6} h_{2}^{-1} h_{3}^{-1} h_{2}^{-1} \\
& =h_{2} h_{1} h_{2}^{-6} h_{3} h_{2} y_{3} h_{3}^{6} h_{2}^{-1} h_{3}^{-1} h_{1}^{-1} h_{2}^{-1} \\
& =h_{1}^{-6} h_{2} h_{1} h_{3} h_{2} y_{3} h_{3}^{6} h_{2}^{-1} h_{3}^{-1} h_{1}^{-1} h_{2}^{-1}
\end{aligned}
$$

Que é equivalente a $h_{1}^{6} y_{1} h_{2} h_{1} h_{3} h_{2}=h_{2} h_{1} h_{3} h_{2} y_{3} h_{3}^{6}$. Analogamente, em $y_{2}=\rho(\beta) y_{2}$ temos

$$
\begin{aligned}
y_{2} & =h_{2} h_{3} h_{2} h_{2}^{-1} h_{3}^{-1} h_{1} h_{2} h_{3} h_{2} h_{2}^{-1} h_{3}^{-1} h_{1}^{-2} y_{1} h_{1}^{2} h_{3} h_{2} h_{2}^{-1} h_{3}^{-1} h_{2}^{-1} h_{1}^{-1} h_{3} h_{2} h_{2}^{-1} h_{3}^{-1} h_{2}^{-1} \\
& =h_{2} h_{1} h_{2} h_{1}^{-2} y_{1} h_{1}^{2} h_{2}^{-1} h_{1}^{-1} h_{2}^{-1} \\
& =h_{2}^{-1} h_{1} h_{2} y_{1} h_{1} h_{2}^{-1} h_{1}^{-1}
\end{aligned}
$$

Que é equivalente a $h_{1} h_{2} y_{1} h_{1}=h_{2} y_{2} h_{1} h_{2}$. E por último em $y_{3}=\rho(\beta) y_{3}$ temos

$$
\begin{aligned}
y_{3} & =h_{2} h_{3} h_{2}^{-1} y_{2} h_{2} h_{3}^{-1} h_{2}^{-1} \\
& =h_{3}^{-1} h_{2} h_{3} y_{2} h_{2} h_{3}^{-1} h_{2}^{-1}
\end{aligned}
$$

Que é equivalente a $h_{2} h_{3} y_{2} h_{2}=h_{3} y_{3} h_{2} h_{3}$. Assim, o grupo $\Gamma(M)$ associado a $M$ é o quociente de $H_{1} * H_{2} * H_{3}$ pelas relações

(1) $h_{i} h_{i+1} h_{i}=h_{i+1} h_{i} h_{i+1}$, para $i=1,2$.

(2) $h_{1} h_{2} y_{1} h_{1}=h_{2} y_{2} h_{1} h_{2}$

(3) $h_{2} h_{3} y_{2} h_{2}=h_{3} y_{3} h_{2} h_{3}$

(4) $h_{1}^{6} y_{1} h_{2} h_{1} h_{3} h_{2}=h_{2} h_{1} h_{3} h_{2} y_{3} h_{3}^{6}$ 
para todo $y_{k} \in H_{k}$, com $k=1,2,3$.

Outro exemplo de interesse é nó Square $L$ (ver figura 5.11).

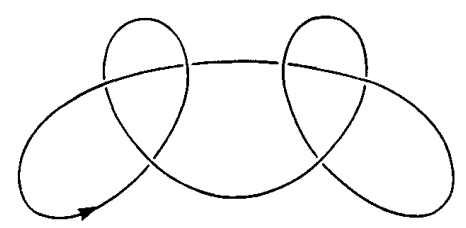

Figura 5.11: O nó Square.

Vamos calcular o grupo $\Gamma(L)$ associado ao nó $L$ de maneira analoga à anterior. Usando o método de [3], pag. 73, obtemos que

$$
L=\widehat{\alpha} \text { onde } \alpha=\sigma_{2}^{3} \sigma_{1}^{-3}
$$

Aqui $\alpha$ é uma 3-trança e portanto $\rho: B_{3} \rightarrow A u t\left(H^{* 3}\right)$ aplica $\alpha \longmapsto \tau_{2}^{3} \circ \tau_{1}^{-3}$. Sabemos pelo trabalho feito anteriormente que

$$
\tau_{1}^{-3}:\left\{\begin{aligned}
y_{1} & \longmapsto h_{2} h_{1} h_{2}^{-3} y_{2} h_{2}^{3} h_{1}^{-1} h_{2}^{-1} \\
y_{2} & \longmapsto h_{2} h_{1} h_{2} h_{1}^{-2} y_{1} h_{1}^{2} h_{2}^{-1} h_{1}^{-1} h_{2}^{-1} \\
y_{3} & \longmapsto y_{3}
\end{aligned}\right.
$$

Agora vamos aplicar $\tau_{2}^{3}$ e assim calcularemos $\Gamma(L)$. Temos,

$$
\begin{aligned}
\tau_{2}^{3} \circ \tau_{1}^{-3}\left(y_{1}\right)= & \tau_{2}^{3}\left(h_{2} h_{1} h_{2}^{-3} y_{2} h_{2}^{3} h_{1}^{-1} h_{2}^{-1}\right) \\
= & \tau_{2}^{2}\left(h_{2}^{-1} h_{3} h_{2} h_{1} h_{2}^{-1} h_{3}^{-3} h_{2} h_{2}^{-1} y_{3} h_{2} h_{2}^{-1} h_{3}^{3} h_{2} h_{1}^{-1} h_{2}^{-1} h_{3}^{-1} h_{2}\right) \\
= & \tau_{2}^{2}\left(h_{2}^{-1} h_{3} h_{2} h_{1} h_{2}^{-1} h_{3}^{-3} y_{3} h_{3}^{3} h_{2} h_{1}^{-1} h_{2}^{-1} h_{3}^{-1} h_{2}\right) \\
= & \tau_{2}^{1}\left(h_{2} h_{3}^{-1} h_{2} h_{2} h_{2}^{-1} h_{3} h_{2} h_{1} h_{2}^{-1} h_{3}^{-1} h_{2} h_{2}^{-3} h_{2} y_{2} h_{2}^{-1} h_{2}^{3} h_{2}^{-1} h_{3} h_{2} h_{1}^{-1} h_{2}^{-1} h_{3}^{-1} h_{2} h_{2}^{-1} h_{2}^{-1} h_{3} h_{2}\right) \\
= & \tau_{2}\left(h_{2}^{-1} h_{3}^{-1} h_{2} h_{3} h_{2} h_{1} h_{2}^{-1} h_{3}^{-1} h_{2}^{-1} y_{2} h_{2} h_{3} h_{2} h_{1}^{-1} h_{2}^{-1} h_{3}^{-1} h_{2}^{-1} h_{3} h_{2}\right) \\
= & h_{2}^{-1} h_{3}^{-1} h_{2} h_{2}^{-1} h_{2}^{-1} h_{3} h_{2} h_{2} h_{2}^{-1} h_{3} h_{2} h_{1} h_{2}^{-1} h_{3}-1 h_{2} h_{2}^{-1} h_{2}^{-1} h_{3}^{-1} h_{2} h_{2}^{-1} y_{3} \\
& h_{2} h_{2}^{-1} h_{3} h_{2} h_{2} h_{2}^{-1} h_{3} h_{2} h_{1}^{-1} h_{2}^{-1} h_{3}^{-1} h_{2} h_{2}^{-1} h_{2}^{-1} h_{3}^{-1} h_{2} h_{2} h_{2}^{-1} h_{3} h_{2} \\
= & h_{2}^{-1} h_{3}^{-1} h_{2}^{-1} h_{3} h_{2} h_{3} h_{2} h_{1} h_{2}^{-1} h_{3}^{-1} h_{2}^{-1} h_{3}^{-1} y_{3} h_{3} h_{2} h_{3} h_{2} h_{1}^{-1} h_{2}^{-1} h_{3}^{-1} h_{2}^{-1} h_{3}^{-1} h_{2} h_{3} h_{2}
\end{aligned}
$$


e

$$
\begin{aligned}
\tau_{2}^{3} \circ \tau_{1}^{-3}\left(y_{2}\right)= & \tau_{2}^{3}\left(h_{2} h_{1} h_{2} h_{1}^{-2} y_{1} h_{1}^{2} h_{2}^{-1} h_{1}^{-1} h_{2}^{-1}\right) \\
= & \tau_{2}^{2}\left(h_{2}^{-1} h_{3} h_{2} h_{1} h_{2}^{-1} h_{3} h_{2} h_{1}^{-2} y_{1} h_{1}^{2} h_{2}^{-1} h_{3}^{-1} h_{2} h_{1}^{-1} h_{2}^{-1} h_{3}^{-1} h_{2}\right) \\
= & \tau_{2}\left(h_{2}^{-1} h_{3}^{-1} h_{2} h_{2} h_{2}^{-1} h_{3} h_{2} h_{1} h_{2}^{-1} h_{3}^{-1} h_{2} h_{2} h_{2}^{-1} h_{3} h_{2} h_{1}^{-2} y_{1}\right. \\
& \left.h_{1}^{2} h_{2}^{-1} h_{3}^{-1} h_{2} h_{2}^{-1} h_{2}^{-1} h_{3} h_{2} h_{1}^{-1} h_{2}^{-2} h_{3}^{-1} h_{2} h_{2}^{-1} h_{2}^{-1} h_{3} h_{2}\right) \\
= & \tau_{2}\left(h_{2}^{-1} h_{3}^{-1} h_{2} h_{3} h_{2} h_{1} h_{2}^{-1} h_{3}^{-1} h_{2} h_{3} h_{2} h_{1}^{-2} y_{1} h_{1}^{2} h_{2}^{-1} h_{3}^{-1} h_{2}^{-1} h_{3} h_{2} h_{1}^{-1} h_{2}^{-1} h_{3}^{-1} h_{2}^{-1} h_{3} h_{2}\right) \\
= & h_{2}^{-1} h_{3}^{-1} h_{2} h_{2}^{-1} h_{2}^{-1} h_{3} h_{2} h_{2} h_{2}^{-1} h_{3} h_{2} h_{1} h_{2}^{-1} h_{3}^{-1} h_{2} h_{2}^{-1} h_{2}^{-1} h_{3}^{-1} h_{2} h_{2}^{-1} y_{3} \\
& h_{2} h_{2}^{-1} h_{3} h_{2} h_{2} h_{2}^{-1} h_{3} h_{2} h_{1}^{-1} h_{2}^{-1} h_{3}^{-1} h_{2} h_{2}^{-1} h_{2}^{-1} h_{3}^{-1} h_{2} h_{2} h_{2}^{-1} h_{3} h_{2} \\
= & h_{2}^{-1} h_{3}^{-1} h_{2}^{-1} h_{3} h_{2} h_{3} h_{2} h_{1} h_{2}^{-1} h_{3}^{-1} h_{2}^{-1} h_{3} h_{2} h_{3} h_{2} h_{1}^{-2} y_{1} \\
& h_{1}^{2} h_{2}^{-1} h_{3}^{-1} h_{2}^{-1} h_{3}^{-1} h_{2} h_{3} h_{2} h_{1}^{-1} h_{2}^{-1} h_{3}^{-1} h_{2}^{-1} h_{3}^{-1} h_{2} h_{3} h_{2}
\end{aligned}
$$

e por último,

$$
\begin{aligned}
\tau_{2}^{3} \circ \tau_{1}^{3}\left(y_{3}\right) & =\tau_{2}^{3}\left(y_{3}\right) \\
& =\tau_{2}^{2}\left(h_{2} y_{2} h_{2}^{-1}\right) \\
& =\tau_{2}\left(h_{2}^{-1} h_{3} h_{2} h_{2}^{-1} y_{3} h_{2} h_{2}^{-1} h_{3}^{-1} h_{2}\right) \\
& =\tau_{2}\left(h_{2}^{-1} h_{3} y_{3} h_{3}^{-1} h_{2}\right) \\
& =h_{2}^{-1} h_{3}^{-1} h_{2} h_{2} h_{2} y_{2} h_{2}^{-1} h_{2}^{-1} h_{2}^{-1} h_{3} h_{2} \\
& =h_{2}^{-1} h_{3}^{-1} h_{2}^{3} y_{2} h_{2}^{-3} h_{3} h_{2}
\end{aligned}
$$

Usando (1) e (2) em $y_{1}=\rho(\alpha) y_{1}$ temos

$$
\begin{aligned}
y_{1} & =h_{2} h_{1} h_{2}^{-1} h_{3}^{-1} h_{2}^{-1} h_{3}^{-1} y_{3} h_{3} h_{2} h_{3} h_{2} h_{1}^{-1} h_{2}^{-1} \\
& =h_{2} h_{1} h_{2}^{-2} h_{3}^{-1} h_{2}^{-1} y_{3} h_{2} h_{3} h_{2}^{2} h_{1}^{-1} h_{2}-1 \\
& =h_{1}^{-2} h_{2} h_{1} h_{3}^{-1} h_{2}^{-1} y_{3} h_{2} h_{3} h_{2}^{2} h_{1}^{-1} h_{2}^{-1}
\end{aligned}
$$

Que é equivalente a $h_{2} h_{3} h_{1}^{-1} h_{2}^{-1} h_{1}^{2} y_{1}=y_{3} h_{2} h_{3} h_{1}^{-1} h_{2}^{-1} h_{1}^{2}$. Usando (1) e (2) em $y_{2}=\rho(\alpha) y_{2}$ temos

$$
\begin{aligned}
y_{2} & =h_{2} h_{1} h_{2} h_{1}^{-2} y_{1} h_{1}^{2} h_{2}^{-1} h_{1}^{-1} h_{2}^{-1} \\
& =h_{2}^{-1} h_{1} h_{2} y_{1} h_{1} h_{2}^{-1} h_{1}^{-1}
\end{aligned}
$$

Que é equivalente a $h_{2} y_{2} h_{1} h_{2}=h_{1} h_{2} y_{1} h_{1}$. E analogamente em $y_{3}=\rho(\alpha) y_{3}$ temos

$$
\begin{aligned}
y_{3} & =h_{2}^{-1} h_{3}^{-1} h_{2}^{3} y_{2} h_{2}^{-3} h_{3} h_{2} \\
& =h_{2}^{-1} h_{3}^{-1} h_{2}^{3} y_{2} h_{3} h_{2} h_{3}^{-3}
\end{aligned}
$$

Que é equivalente a $h_{3} h_{2} y_{3} h_{3}^{3}=h_{2}^{3} y_{2} h_{3} h_{2}$. Assim, o grupo $\Gamma(L)$ associado a $L$ é o quociente de $H_{1} * H_{2} * H_{3}$ pelas relações

(1) $h_{i} h_{i+1} h_{i}=h_{i+1} h_{i} h_{i+1}$, para $i=1,2$.

(2) $h_{1} h_{2} y_{1} h_{1}=h_{2} y_{2} h_{1} h_{2}$

(3) $h_{2}^{3} y_{2} h_{3} h_{2}=h_{3} h_{2} y_{3} h_{3}^{3}$ 
(4) $h_{2} h_{3} h_{1}^{-1} h_{2}^{-1} h_{1}^{2} y_{1}=y_{3} h_{2} h_{3} h_{1}^{-1} h_{2}^{-1} h_{1}^{2}$

para todo $y_{k} \in H_{k}$, com $k=1,2,3$ 


\section{Índice Remissivo}

apresentação, 11

finita, 11

base, 9

complexo CW, 58

conjunto

das consequências, 11

de geradores, 11

de relatores, 11

de relatores definidores, 11

consequência, 11

Dehn

meia torção de, 87

espaço

contrátil, 51

simplesmente conexo, 51

framing, 77

preferido, 78

grupóide, 22

árvore, 24

conexo, 25

discreto, 24

grupo

de um enlaçamento, 76 finitamente apresentado, 11

grupo livre, 9

homotopia

de caminhos, 47

de funções, 50

equivalencia de, , 51

isotopia, 52

ambiente, 53

relativa, 52

letras, 6

livre sobre, 4

longitude, 77

monóide, 5

livre, 5

Multiplicação Parcial, 21

Nó

Granny, 106

Square, 108

Trevo, 104

número de enlaçamentos, 78

palavra, 6

comprimento, 6

posto, 9 
Push out

grupóides, 29

grupos, 17

redução, 6

elementar, 6

relação, 11

Representação de grupo, 100

fiel, 100

retrato, 54

por deformação, 54

símbolos geradores, 11

subgrupóide, 24

completo, 24

Teorema

Alexander, 71

da apresentação de Artin, 65

da representação de Artin, 65

forma normal para grupos livres, 8

Markov, versão algébrica, 75

Markov, versão geométrica, 74

Seifert-Van Kampen, 53

Von Dyck, 12

vizinhança tubular, 77 


\section{Referências Bibliográficas}

[1] D. E. Cohen - Combinatorial Group Theory: A Topological Approach, Cambridge University Press, 1989.

[2] J. S. Birman - Braids, Links and Mapping Class Groups, Annals of Math. Studies 82, Princeton University Press, 1973.

[3] V. L. Hansen - Braids and Coverings: Selected Topics, Cambridge University Press, 1989.

[4] W. S. MASSEY - Algebraic Topology: An Introduction, Harcourt, Brace and World Inc., 1967.

[5] JOHn CRISP AND LUIS PARIS - Representations of the braid group by automorphisms of groups, invariants of links and Garside group, Pacific Jornal of Mathematics, vol. 221, número 1, 1-28, setembro 2005.

[6] Dale Rolfsen - Knots and Links, AMS Chelsea Publishing, 1976.

[7] V. ShPILRAIN - Representing braids by automorphisms, Internat. J. Algebra Comput. 11:6 (2001), 773-777. MR 2003a:20061 Zbl 1024.20036

[8] M. WADA - Group invariants of links, Topology 31:2 (1992), 399-406. MR 94e:57014 Zbl 0758.57008

[9] P. Dehornoy - Braids groups and left distributive operations, Trans. Amer. Math. Soc. 345:1 (1994), 115-150. MR 95a:08003 Zbl 0882.20021

[10] P. Dehornoy - A fast method for compating braids, Adv. Math. 125:2 (1997), 200235. MR 98b:20060 Zbl 0882.20021 